\title{
SELF-REGULATION AND SOCIAL INFLUENCE: A LIMITED-RESOURCE ACCOUNT OF RESISTING AND YIELDING TO PERSUASION
}

\author{
LOES JANSSEN
}


Thesis, University of Twente, 2010

(c) Loes Janssen

ISBN: 978-90-365-2971-6

Cover design and lay-out by Emiel L.C. van Vilsteren

Printed by Ipskamp Drukkers B.V., Enschede, the Netherlands

The research presented in this thesis was funded by a grant from the Netherlands Organisation for Scientific Research (NWO) 


\section{SELF-REGULATION AND SOCIAL INFLUENCE: A LIMITED-RESOURCE ACCOUNT OF RESISTING AND YIELDING TO PERSUASION}

\section{PROEFSCHRIFT}

ter verkrijging van

de graad van doctor aan de Universiteit Twente, op gezag van de rector magnificus,

prof. dr. H. Brinksma,

volgens besluit van het College voor Promoties

in het openbaar te verdedigen

op vrijdag 12 maart 2010 om 15.00

door

Loes Janssen

geboren op 20 juli 1981

te Nijmegen 
Dit proefschrift is goedgekeurd door de promotor: Prof. dr. A.Th.H. Pruyn en de assistent-promotor: Dr. B.M. Fennis 


\title{
Samenstelling promotiecommissie
}

\author{
Promotor: $\quad$ Prof. dr. A.Th.H. Pruyn \\ Assistent-promotor: Dr. B.M. Fennis \\ Leden: $\quad$ Prof. dr. J.W.J. Beentjes \\ Prof. dr. M. Junger \\ Dr. C. Martijn \\ Prof. dr. D.A. Stapel \\ Prof. dr. ing. W.B. Verwey
}





\section{Contents}

Chapter 1 General Introduction:Towards a Two-Stage Model

Chapter 2 Stage One: Weakening the Ramparts: Actively Responding to an Influence Attempt Induces Self-Regulatory Resource Depletion

Chapter 3 Stage Two: The Path of Least Resistance: Self-Regulatory 59 Resource Depletion Affects Compliance through Heuristic Decision Making

Chapter 4 Extending the Model: Forewarned is Forearmed:

Conserving Self-Control Strength to Resist Social Influence

Chapter 5 General Discussion

References

Appendix

Samenvatting (Summary in Dutch) 165

Dankwoord (Acknowledgements in Dutch) 



\section{Chapter 1}

General introduction: Towards a two-stage model 

magine, on a Saturday in spring you are on a shopping trip with some friends, in the city centre of your favorite town. As your friends dive into another store, you decide to take it easy and wait outside until they return. Taking a break, enjoying the spring sunshine, you notice a young man walking up to you. He is good-looking, wears a nice suit, and broadly smiling he starts some small talk about the weather. You cannot immediately figure out what he is up to, when he asks you about your interest in reading books. "May I ask you whether you enjoy reading? What kind of books do you like? Do you buy your own books, or do you prefer going to the library? How often do you buy a new book?"You chat about this topic for a while, a bit reluctant at first, but then where is the harm in chatting with a friendly guy on a sunny afternoon about one of your favorite pastimes? Your friends should be returning from the store any time now, and you can end this conversation whenever you want to. After discussing your interest in books, the young fellow would also like to know about your interest in movies and music. "Do you like seeing movies, out in a theatre, or do you rather stay at home? Do you buy DVDs often, or do other members of your family? Do you own a large CD collection? What kind of music do you like?" Rather innocent questions, no trouble answering for a straightforward modern intellectual with a healthy interest in the latest literature, hottest pop music, and modern cinema. "Well then", the young man says, "I think I have the best offer you ever encountered!" A lingering suspicion reaches consciousness: a good old sales pitch, how could you have missed that? "I happen to be a representative of book club $X$, and when you join us you will receive an astonishing discount on the newest books, CDs, and DVDs, and as a very special welcome gift you can pick out five of them for free!"Though your gut feeling tells you to be on guard and think this through before making a decision, the offer sounds rather appealing since you just showed your interest in these products. Moreover, the man appears to be very friendly, trustworthy and convincing, the sun is shining, why not? Before you know it you have signed a form, your friends are coming out of the store, the young man is busy talking to someone else, and you continue your shopping trip as the latest member of book club X. A few weeks later you notice that the club's registration fee has been withdrawn from your 
bank account and you have to decide which products to order from the club catalogue, as you contracted to do a prescribed number of times per year. Now you come to think of it, you actually prefer to decide for yourself where and when to buy a book, $C D$, or movie disc, and you realise that the offer was not that attractive after all. You think again about what happened that Saturday afternoon, and wonder how you could have been so mindlessly compliant. What happened to your autonomous will, your by nature so balanced and controlled self?

This dissertation focuses on the internal process that takes place when people are being confronted with influence situations as the one presented above. A situation which is characterized by an influence agent - most likely a compliance professional like a sales representative, marketer, or fundraiser - persuading a target of influence into complying with a request, such as purchasing a product, subscribing to a service, or donating money to a charity organization. Although a predominant part of these requests is unsolicited, and initially received with a skeptical response, influence agents are often stunningly successful in eliciting compliance, and manage to urge their targets to respond in their desired way. As consumers, people are constantly persuaded to invest time, effort, or money supporting causes and organizations they have sometimes never heard of before, without necessarily expecting a return on their investment. People accept offers they were not planning to yield to in advance, often wondering later on why they fell for it. An intriguing question to ask, therefore, is what makes people comply with these types of persuasive requests without any overt pressure. What makes it so hard to say "No" when confronted with an (unwanted) influence attempt? And given that knowledge, what determines whether people succeed at resisting persuasion?

The research presented in this dissertation approaches these questions from a selfregulation perspective. Specifically, it suggests that one key feature of many influence situations is that they wear down people's self-control resources. Resisting persuasion is argued to require active self-regulation, and when resources for self-regulation are low, 
one's attempts at resistance are more likely to fail. Hence, people's success in dealing with (unwanted) persuasion is expected to depend for an important part on the availability of resources to actively control the self. In line with this notion, the research in this dissertation also demonstrates that when people anticipate persuasion, they become more efficient in allocating their remaining regulatory energy. When resources for selfregulation are low, people start conserving their remaining self-control resources to be able to resist future persuasion, which proves to be a successful (unconscious) strategy to resist a persuasive appeal. By adopting a self-regulation perspective, this dissertation aims to point out a key mechanism responsible for the effectiveness of many social influence situations, thereby contributing to the understanding of the dynamics behind resisting and yielding to persuasion.

In the remainder of this chapter, the theoretical background of the research in this dissertation will be discussed in more detail. Starting with an introduction to the field of social influence, the focus will be on the topic of behavioral compliance and its empirical evidence relevant to the present research. Next, a prevalent theory of self-regulation, the 'limited-resource model of self-control' (Baumeister, Bratslavsky, Muraven, \& Tice, 1998; Vohs \& Heatherton, 2000; Baumeister, Vohs, \& Tice, 2007) will be discussed and linked to consumer behavior. Finally, a two-stage model representing a limited-regulatory resource perspective on social influence will be introduced, which provides the basis for the studies in this dissertation. The chapter finishes with an overview of the subsequent chapters. 


\section{Social influence: compliance with persuasive requests}

Social influence, the process of changing our attitudes, feelings and behaviors in response to intentional and sometimes unintentional actions of others, has been fascinating scholars for over half a century. Among the most prominent lines of research in this field are classic studies on conformity and social norms (Asch, 1951, 1956; Deutsch \& Gerard, 1955; Sherif, 1936), obedience (Milgram, 1963, 1974), persuasion and attitude change (Chaiken, 1980; Petty \& Cacioppo, 1981, 1986), and compliance (Burger, 1986; Cialdini, 1993; Cialdini, Cacioppo, Bassett, \& Miller, 1978; Freedman \& Fraser, 1966). In contrast to the classic social influence studies, where the targets of influence were confronted with explicit social forces that were well within conscious awareness, scholars in recent years have increasingly emphasized processes that are subtle, indirect, and outside conscious awareness of the target of influence (Cialdini \& Goldstein, 2004).

One of the main research areas to which these subtle processes apply is the study of behavioral compliance, or acquiescence to persuasive requests. The process of yielding to compliance plays a role in many types of social interaction, and pre-eminently within the field of marketing communication and consumer psychology where it has become a central topic of study. Within this field it is highly relevant to study what makes consumers comply with several types of (unsolicited) requests, usually without overt pressure. What makes people subscribe to a book club, buy special bargain hotel vouchers, or change their energy supplier when accosted by sales representatives on the street, in a store, or even at home? Offers that they were not planning to yield to in advance and, as it seems, they could just as well refuse. And similarly, what makes people sign a petition, donate money to charity, or volunteer on behalf of an unknown organization or cause? How do persuasion agents representing profit and non-profit organizations manage to persuade individuals to perform a desired behavior, without these individuals necessarily being interested in their products and services, or feeling positive about charitable giving? Typically, these compliance professionals do not bluntly 
ask for a donation of time or money, but will embed their target request in a scripted social influence technique, which is a tactic specifically designed to increase the odds of yielding to a request. Decades of studies on social influence confirm that consumers are induced to comply with a request at much higher rates when approached with a social influence technique than when the request is made without a scripted warm-up period (Burger, 1999; Cialdini \& Goldstein, 2004).

The variety of these strategies as well as their potential to change people's behavior is remarkable. One of the most extensively studied social influence techniques is the foot-in-the-door technique (Freedman \& Fraser, 1966). Administering this technique, an influence agent will initially present an individual with a small request that is difficult to refuse, followed by a more substantial request, which is the target request that the agent has set out to gain compliance with. For example, imagine a fundraiser who approaches you in the street and asks whether you are willing to answer a few questions about your attitude towards charitable giving. You answer these seemingly harmless questions and then this person asks you to subscribe to the charity program he is working for. Metaanalyses suggest that the chance that one agrees to this request is now significantly larger than if the fundraiser had asked for a contribution right away (Burger, 1999; Fern, Monroe, \& Avila, 1986).

Another successful and frequently studied social influence technique is the door-inthe-face technique (Cialdini et al.,1975). This technique starts off with a relatively large request that is likely to be rejected, and after this a milder target request is posed. For example, a group of girl scouts calls at your house and requests you to buy a dozen raffle tickets to support their club. You kindly mention that a dozen would be way above your budget, on which they propose that you then buy a single ticket instead. Studies suggest that the chance that one buys a single raffle ticket substantially increases when one previously rejected the request to buy a dozen (for a review, see O'Keefe \& Hale, 1998, 2001). Other social influence techniques that have been topics of investigation 
include the low-ball procedure (after obtaining a commitment to an offer it is made less desirable, Burger \& Petty, 1981), the that's-not-all technique (an initial offer is improved before the target can respond, Burger, 1986; Pollock, Smith, Knowles, \& Bruce, 1998), and the disrupt-then-reframe-technique (a small disruption in the request is followed by a direct persuasive reframing of the request, Davis \& Knowles, 1999; Fennis, Das, \& Pruyn, 2004, 2006).

But how and why do these techniques promote compliance? Recently, Cialdini and others (e.g., Cialdini, 1993; Cialdini \& Goldstein, 2004) have forwarded the principle of automaticity as the cornerstone of all influence techniques. According to these scholars, the effectiveness of most techniques hinges on the notion of automaticity or 'mindlessness' (Langer, 1992). Instead of carefully scrutinizing the merits of a request or offer, people appear to respond mindlessly (i.e., with little conscious effort) when confronted with a social influence technique. Under these conditions of reduced mental alertness, people are thought to fall back on habit and routine and are prone to employ 'mental shortcuts' or simple heuristics for deciding how to act. Use of these heuristics will generally increase the likelihood of compliance (Cialdini, 1993)

As such, it has been generally assumed that the foot-in-the-door technique is effective because it mindlessly triggers people's desire to be or appear consistent with prior commitments (Burger, 1999). In the example at the beginning of this chapter one (publicly) declared one's interest in reading, which rendered it more difficult to decline a subsequent discount offer of a book club. Apparently one feels committed to and wants to be consistent with what one has already said. People are also thought to engage in a self-perception process after seeing themselves agreeing with the initial request, and apparently infer from this that they are'the kind of person to comply with these kinds of requests.' For example, stating to a fundraiser that you have a positive attitude toward charitable giving will likely induce the self-perceived notion that you are the kind of person that supports charities. This highly salient self-perception is then assumed to 
function as a decisional heuristic, increasing the odds of compliance with the more substantial target request, like donating money to the charity in question. Another social influence technique that taps into the principles of commitment and consistency is the low-ball procedure (Cialdini et al., 1978; Burger \& Petty, 1981). The essence of this procedure is that one is presented with an initial request (e.g., "May I invite you to give a guest lecture at my university?"), and after one has complied and has committed oneself to the deal, the cost of compliance is raised ("The lecture is scheduled for the first period, so you'll have to show up at 8.00 a.m."). The act of initial compliance is supposed to create a commitment and activates the principle of consistency, which in turn fosters compliance with the intended target request.

The most widely accepted psychological explanation for the door-in-the-face technique is that it hinges on the norm of reciprocity, i.e. the felt obligation to return favors (Gouldner, 1960). Specifically, Cialdini et al. (1975) explained this rejection-thenmoderation procedure in terms of reciprocal concessions: the influence agent makes a clear concession by downsizing the initial request (after your refusal to buy a dozen raffle tickets the girl scouts propose that you buy a single ticket instead), which evokes the need for the target of influence to make a concession in return and therefore to comply with the milder request (you buy that single ticket).

Other strong decisional rules or heuristics that people resort to in situations of influence are liking (people generally agree with people they like), social proof (since others do it, it will probably be the correct thing to do), authority (people generally agree with people that are - affiliated with - a highly credible source), or scarcity (the availability of an offer is limited, and therefore appears to be more valuable; cf. Cialdini's principles of influence, 1993).

In sum, this variety of procedures aimed at eliciting some kind of acquiescence seem to have in common that they induce people to act according to some automatic, fixed 
action pattern, in which they do not carefully weigh up the pros and cons of a request or offer but mindlessly act upon some basic heuristic principle, increasing the odds of compliance with the target request. An appropriate question to ask, and the question that is key to the research in this dissertation, is what produces the mindlessness in these influence contexts? Why do people proceed with a minimum of cognitive effort or thought and behave automatically, falling back on heuristics for decision making, when confronted with an influence technique? Although automaticity has been proffered as a basic requirement for the techniques to work, no study to date has directly addressed this key question. An examination of the literature reveals that automaticity has not been measured directly but rather inferred from indirect manipulations (e.g., Langer, Blank, \& Chanowitz, 1978; Pollock et al., 1998).

The research in this dissertation argues that the origins of this mindlessness can be found in a characteristic that almost all successful influence techniques have in common: multiple decision moments or sequential requests (Fern et al., 1986). That is, the target of influence has to yield to one or several initial request(s), answer probing questions, or make choices before the target request is presented. It is proposed that the preliminary stage of these sequential request techniques triggers one underlying psychological mechanism that accounts for the impact on subsequent compliance: self-regulation failure brought about by self-regulatory resource depletion (Baumeister et al., 1998; Vohs \& Heatherton, 2000; for a review, see Baumeister, Vohs, \& Tice, 2007). It is argued that consciously attending and actively responding to the initial requests of an influence attempt drains the self's finite regulatory resources. The active self becomes weakened, a state that paves the way for subsequent acquiescence due to a lack of regulatory resources available to refuse the target request. Before presenting a two-stage model which represents a limited-regulatory resource account of the impact of social influence techniques, an overview will be given of prevalent research in the area of self-regulation and its significance to consumer behavior. 


\section{A theory of self-regulation: self-control capacity as a limited resource}

Self-regulation, or an individual's ability to override, interrupt, and otherwise alter its own responses, is a central aspect of human functioning (Muraven, Tice, \& Baumeister, 1998). Although presumably the greater part of human behavior is influenced by automatic or nonconscious processes and does not involve preplanned or deliberate control by the self (Bargh, 1997), being able to consciously and actively control the way you think, feel, or behave is essential for an individual to achieve its goals. Regulating the self is involved in many crucial functions, such as making choices and decisions, initiating and inhibiting behavior, and making and carrying out plans of action (Baumeister et al., 1998). Self-control is related to success in many spheres of life, such as being able to keep on a diet, resisting temptations, managing emotions, breaking bad habits, and finishing difficult tasks (Muraven et al., 1998). Self-regulation is not only important in the personal sphere, but also has an essential role in interpersonal functioning. Successfully maintaining relationships, gaining social acceptance, and managing impressions in dyadic interactions all require active regulation by the self (Vohs, Lasaleta, \& Fennis, in press).

Over the years, the concept of self-regulation has been approached and defined in several ways (for an overview, see Baumeister \& Vohs, 2004). The present dissertation adopts a view of self-regulation as operating on the basis of a limited resource that resembles strength or energy. Despite self-regulation's vital role, there is increasing evidence pertaining to the idea that the self's capacity for active volition is limited (Baumeister et al., 1998). Similar to the functioning of a muscle, the limited-resource model of self-control (Baumeister et al. 1998; Vohs \& Heatherton, 2000; for a review see Baumeister, Vohs, \& Tice, 2007) posits that any behavior that involves deliberate and regulated responses by the self draws on a limited intrapsychic resource, akin to strength or energy. Any act of volition is posited to have a detrimental impact on any subsequent act of volition due to the fact that they must share the same limited (and dwindling) 
resource. As a consequence, and similar to muscle failure after straining, a series of self-regulatory acts will deplete people's self-regulatory energy to the point of selfregulatory failure (Baumeister, Schmeichel, \& Vohs, 2007). In this state of self-regulatory resource depletion (or 'ego depletion'), the controlled, purposeful self fails to function effectively, which renders people vulnerable to untoward impulses, habit, routine, and automatic processes (Baumeister \& Vohs, 2007; Vohs, Baumeister, \& Ciarocco, 2005), all key indicators of mindlessness.

In line with the statements of the limited-resource model of self-control, in this dissertation the terms 'self-regulation' and 'self-control' will be used interchangeably. Furthermore, whereas the ability to perform self-control is for an important part determined by situational demands, it is dependent on individual differences as well (Tangney, Baumeister, \& Boone, 2004). Although individual differences in dispositional self-control ability were also considered (Chapter 3, Experiment 3.4), this dissertation mainly focuses on situational differences in self-control.

Many studies have shown that performing a (brief) preliminary act of self-control undermines self-regulation on a subsequent, unrelated task. In a classic study by Baumeister et al. (1998), participants were seated at a table with a stack of chocolate chip cookies and a bowl of radishes placed in front of them. Ostensibly to study taste perception, half the participants were to taste the cookies, but leave the radishes, and the other half of participants were to taste the radishes, but was not allowed to eat the cookies. Participants who had to force themselves to eat the radishes instead of the tempting chocolate cookies subsequently quit faster on unsolvable figure tracing puzzles than participants who did not have to exert self-control over eating. In a comparable study by Muraven et al. (1998), participants were told to write down all their thoughts. They were either instructed to think about a white bear as much as they could, instructed not to think about a white bear, or they were given no special thought control instructions. Participants who had to suppress thoughts about a white 
bear subsequently quit sooner on unsolvable anagrams than participants in the control conditions. Furthermore, Vohs and Heatherton (2000) showed that chronic dieters who exerted self-control over their facial expressions and emotional reactions were less able to inhibit their subsequent intake of ice cream during a taste-and-rate task than participants who were allowed to let their emotions flow naturally. Finally, experiments by Schmeichel, Vohs, and Baumeister (2003) demonstrated an impairment in intelligent functioning, such as logic, reasoning and active problem solving, for participants who had previously regulated their attention or their emotional responses while watching a video. Importantly, as compared to these relatively complex forms of cognitive activity, a state of self-regulatory resource depletion did not negatively affect simpler forms of information processing, such as retrieving general knowledge from memory or following well-learned rules (such as basic arithmetical computations). Hence, the conscious, active self appears to be vital for some mental acts but not for others. However, the authors conclude that irrespective of the complexity of a task, the essence is the extent to which the task requires high-level cognitive control and active guidance by the self.

The notion that active guidance by the self is costly has been demonstrated by several other studies as well. Vohs et al. (2008) demonstrated that participants who made a series of choices and decisions (e.g., regarding consumer products) showed poorer self-regulation afterwards as compared to people who viewed or rated similar options without making choices. An initial act of self-regulation also rendered people less inclined to make active responses and more prone to favor a passive response option (Baumeister et al., 1998). Furthermore, Vohs et al. (2005) demonstrated that depletion of regulatory resources impaired effective self-presentation in dyadic interactions and lead to falling back on habitual, overlearned patterns of selfdisclosure. Across these studies there is no evidence that resource depletion effects can be explained by mood or emotion, or to a feeling of having already done enough for the experiment (Baumeister, Sparks, Stillman, \& Vohs, 2008). Interestingly, recent research by Gailliot, Baumeister, and DeWall (2007) has suggested that self-control 
performance literally relies on a limited energy resource, namely, blood glucose. In their studies, acts of self-control caused glucose levels to drop, and low levels of blood glucose predicted impaired performance on subsequent self-control tasks. However, consuming a glucose-containing drink appeared to eliminate those impairments.

Notwithstanding the multiple observations of impaired self-control performance after an initial self-control task, research has supported the notion that a depleted state does not reflect a complete exhaustion of resources but merely a temporary or relative deficit. Hence, people can still self-regulate to a certain extent when they are in a depleted state, provided that they are motivated to do so. Motivational concerns thus are able to function as a buffer against the detrimental effects of self-regulatory resource depletion. In support of this hypothesis, research by Muraven and Slessareva (2003) showed that depleted participants who were given an incentive (e.g., money) to exert self-control performed as well as non-depleted participants. Other ways to overcome depletion that have been documented in the literature are forming implementation intentions (circumventing the need for cognitive control by specifying in advance how one will act in a certain situation), or priming people with a person exemplar that represents perseverance (Martijn et al., 2007). Moreover, 'replenishment' of diminished resources has been procured by inducing positive affect (Tice, Baumeister, Shmueli, \& Muraven, 2007) and by affirming the self's core values (Schmeichel \& Vohs, 2009), which improved self-regulation for people whose resources had previously been depleted. However, these short-term antidotes carry a cost, since exerting selfcontrol after being already depleted will ultimately drain the resource to a critical extent, leaving an individual much more depleted afterward (Baumeister et al., 2008).

Acknowledging the motivational aspects of self-regulation, Muraven, Shmueli, and Burkley (2006) stated that the 'classic' depletion effect should be viewed as an effort to conserve what is left of a diminished resource. In three studies they showed that expecting to exert self-control in the near future motivated participants who exerted 
self-control in the past (and were thus depleted of their regulatory resources) to conserve their remaining self-control strength for this future task. These participants performed worse on an intervening measure of self-control than participants who were either not depleted, or not expecting future self-control. Moreover, when performance on this future task was actually measured, participants who were initially depleted but conserved resources performed as well as non-depleted participants. In contrast, initially depleted participants not expecting to exert self-control in the future performed worse than participants in the other conditions.

In sum, we should state that self-control performance under depletion conditions is amenable to short-term modulation as a function of motivation, and the motivation to engage in self-regulation is therefore an important component of self-control as well (Baumeister \& Vohs, 2007). A state of self-regulatory resource depletion and the expectation of future self-control demands may cause people to become more selective in exerting self-control. People are likely to be involved in a constant trade-off between multiple self-control demands, and motivation can compensate for a reduced ability to self-regulate. As Muraven and Slessareva (2003) already stated, this trade-off is not necessarily a conscious and deliberative process, but rather something individuals do continually with very little awareness, in contrast to the exertion of self-control itself, which is typically an act of conscious volition.

An alternative perspective on the self-regulatory strength model has recently been put forward by Dewitte, Bruyneel, and Geyskens (2009). Starting from an alternative cognitive control model, they claim that the strength-model only holds when the control processes that have been recruited to deal with the demands of a first selfregulatory task differ from the control processes needed for a second task. Depletion effects thus should only occur when the control processes required for two consecutive tasks differ, since in between people will have to adapt to the temporary misfit between their cognitive system and the demands of the task at hand. Indeed, Dewitte et al. (2009) 
demonstrated that when two consecutive self-regulatory situations required similar control processes (e.g., restraining food intake), initial engagement in self-regulation did not impair, but instead enhanced subsequent self-regulation. However, when two consecutive self-control tasks required different control processes (e.g., restraining food intake and solving an anagram), the 'classic' depletion effect did occur.

In recent years, these insights into the self's executive function are being more and more applied to explain several types of consumer behavior. Within this field, selfcontrol has been primarily linked to the capacity to resist temptations and impulses (Baumeister, 2002; Faber \& Vohs, 2004). For example, Vohs and Faber (2007) showed that a lowered level of self-control resources caused an increase in impulsive spending. In their studies, depleted participants were willing to spend more money on a variety of consumer goods, and actually spent more money in a mock store than participants who had not earlier engaged in self-control. Another study by Bruyneel, Dewitte, Vohs, and Warlop (2006) showed that participants who had to make a series of product choices during a mock shopping trip, which depleted their regulatory resources, became more vulnerable to the temptation of salient affective product features at the cost of cognitive product features: they favored a more attractive but more expensive type of candy over a less attractive but cheaper type of candy, as compared to participants who followed a shopping list. Finally, in the consumer realm, Baumeister (2002) has argued that due to a gradual depletion of the self's regulatory resources during the course of the day, one is likely to purchase more impulsively and spend more money later in the day. Similarly, one could predict that the more time shoppers spend at the mall, the more depleted they get, and therefore the more money they spend at the final store. Hence, it has been hypothesized and demonstrated that consumers have a tendency to become more mindless, impulsive and less self-controlled toward the end of a series of self-control acts within an influence context. 


\section{A limited-resource perspective on social influence}

In sum, research demonstrates that the self's capacity for active self-regulation is limited. A series of self-regulatory acts depletes one's resource of mental energy, and in a state of self-regulatory resource depletion the self resorts to more passive and loweffort courses of action, is more likely to yield to temptation, and relies more on habit, routine, and automatic processes (Baumeister, 2002; Baumeister, Muraven, \& Tice, 2000; Vohs et al., 2005). To the extent that responding to the initial requests of compliancegaining procedures involves deliberate, conscious, and controlled self-regulation, it is plausible that situations that promote these types of initial responses would induce selfregulatory resource depletion. Consumers' self-control resources are likely to be taxed in the initial request-phase of an influence attempt, when answering several involving questions, agreeing to receive and process a persuasive message, or, conversely, resisting or rejecting an opening offer (as in a door-in-the-face script). The resulting state of self-regulatory resource depletion has been shown to lower resistance to persuasion, presumably because it hinders the processing of message-relevant information (Burkley, 2008; Wheeler, Briñol, \& Hermann, 2007). More specifically, Wheeler et al. (2007) showed that depleted participants became less sensitive to the quality of the arguments in a counterattitudinal persuasive message than their non-depleted counterparts. Hence, in contrast to non-depleted participants, depleted participants did not distinguish between strong and weak arguments, and were even more persuaded by weak arguments than non-depleted participants. Since a state of self-regulatory resource depletion decreases sensitivity to argument quality, then following dual-process logic and by the same token, it should increase the weight on heuristics present in the influence context. The implications stemming from dual-process frameworks (e.g., Chaiken \& Trope, 1999) are highly relevant in the present context since heuristics are typically present in influence settings (e.g., the principle of reciprocity features in the door-in-the-face technique, the foot-in-the-door and the low-ball techniques are based upon the principles of commitment and consistency). Since these heuristics generally point to compliance as an 
efficient behavioral response, a state of self-regulatory resource depletion is likely to result in increased levels of compliance. Hence, to sum up, it was hypothesized that yielding to the initial request-phase of a compliance-gaining procedure, consisting of multiple, sequential requests, taxes self-control resources. The resulting state of self-regulatory resource depletion will enhance the weight on heuristic processing and increase the odds of compliance with the target request of the influence technique. It is important to note that depletion will not increase compliance by default: it is only expected to increase the odds of yielding to a request when a heuristic is present in the influence context that promotes compliance. These hypotheses are represented in a two-stage model as pictured below.

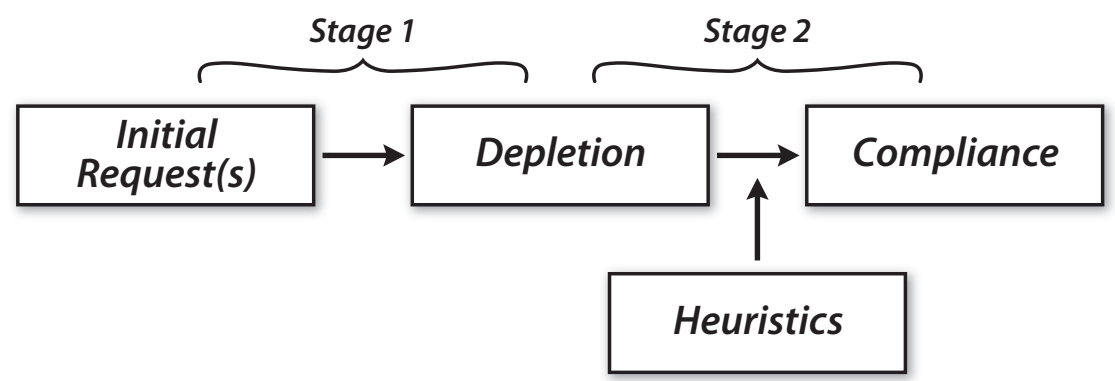

Figure 1. A self-regulatory resource depletion account of the impact of sequential request techniques (adapted from Fennis, Janssen, \& Vohs, 2009).

This model claims that self-regulatory resource depletion is an important underlying psychological mechanism that accounts for the impact of sequential request techniques that are being used by influence professionals to elicit consumer compliance. It claims that the mindlessness so often observed in influence situations has its roots in the influence setting itself, and can be defined as a depletion-induced reliance on heuristics. In the following chapters of this dissertation (Chapters 2 and 3), both stages of the model will be elaborated further, and supported by empirical evidence from a series of both lab and field experiments. 
In Chapter 4, empirical evidence is presented that extends the claims made by the two-stage model. The demonstration that self-regulation failure may be at the base of yielding to compliance implies that successfully resisting persuasion will depend for an important part on the availability of resources to actively control the self. Hence, whereas a low level of self-control resources increases susceptibility to influence, a high level of regulatory resources likely increases the chance that one is able to resist an influence attempt. But does this mean that all is lost for those with low self-control? Does a temporary lowered level of self-regulatory resources automatically imply a weakened defense against an influence attempt? The role of motivation is argued to be key here. The research in Chapter 4 proposes that individuals low in self-control resources can still be successful at defending themselves against an unwanted persuasive attack, when prompted to be efficient in allocating their remaining self-regulatory resources. Based on the notion that a depleted state does not reflect a complete exhaustion of resources but merely a temporary or relative deficit (Muraven et al., 2006), it is proposed that initially depleted people can still be successful at resisting persuasion when they are motivated to temporarily economize on their use of self-control resources. Specifically, a forewarning of an upcoming influence attempt should prompt these individuals to conserve their remaining resources (by letting their self-control performance temporarily suffer) to enable effortful resistance at a later stage. A forewarning is thus expected to function as a motivational factor that stimulates people with low self-control ability to save up their remaining self-control energy to be able to avoid future persuasion.

In sum, the outcome of a social influence process will for an important part be determined by people's ability to exert self-control over their cognitive and behavioral responses, but also by their motivation to engage in self-control, and consequently by their efficiency in allocating their self-control resources. Whereas Chapters 2 and 3 focus on the role of self-control ability in social influence situations, and point to compliance as a consequence of this ability being low, Chapter 4 highlights the motivational aspects of self-control. Chapter 4 stresses resistance as a more likely outcome of a social 
influence process when self-control ability is high, or when one is motivated to become efficient in the use of remaining resources when self-control ability is not optimal. By addressing the ability and motivational aspects of self-control, and focusing on the role of self-regulation in both compliance with as well as resistance to persuasive requests, this dissertation provides an integrative perspective on the dynamics behind resisting and yielding to social influence.

In Chapter 5, the theoretical and practical implications and contributions of the empirical findings, as presented in Chapters 2 - 4, will be discussed, as well as some limitations and directions for future research. To conclude the present chapter, an overview of the three empirical chapters of this dissertation is presented below. All these chapters are based on articles that have been published or have been submitted for publication in academic journals. Since these articles were written in collaboration with others, the authors will be referred to as 'we'. The chapters can be read independently from each other, therefore there may exist some overlap. 


\section{Overview of the empirical chapters}

In Chapter 2, the first stage of the two-stage model as presented in the current chapter (Figure 1) will be elaborated and tested. Four experiments will be reported, that were either conducted in the lab or in a more naturalistic setting, showing that actively responding to the initial request-phase of a sequential request social influence technique taxes self-regulatory resources, resulting in a state of self-regulatory resource depletion. In each of the four experiments, participants are presented with an initial request, consisting of a series of involving questions that entail either (a) active self-presentation or (b) demanding cognitive operations (cf. Freedman \& Fraser, 1966). These processes are argued to be specific attributes of successful sequential request procedures and are known to elicit self-regulatory resource depletion. Depletion is witnessed on a diverse range of measures that have been widely used in self-control research (Baumeister et al., 1998; Vohs et al., 2005). Moreover, several alternative explanations for the effect of an initial request on self-regulatory resource depletion are tested and refuted. Results show that being confronted with an initial request consisting of self-presentational or cognitively demanding questions does not affect emotions, liking for the influence agent, or perceptions of receiving too many demands from the requester. Nor can the effects be attributed to differences in duration of the conditions, or simply having an unanticipated conversation on the street with an unknown person. Finally, the research in Chapter 2 bridges Stage 1 and Stage 2 of our model in showing that self-regulatory resource depletion functions as a mediating variable between the response to the initial request and the response to the target request of a sequential request technique.

As a direct extension of Chapter 2, Chapter 3 focuses on the second stage of the twostage model as presented in the current chapter (Figure 1). Four experiments will be reported, that were either conducted in the lab or in a field setting, showing that a state of self-regulatory resource depletion fosters the use of salient heuristics, when present in the persuasion context, which increases the chance of compliance with a 
request (the target request of a social influence technique). Self-regulatory resource depletion is either induced with a self-control task adopted from previous self-control research, or people's dispositional self-control is measured (being either high or low). Participants are presented with a diversity of salient heuristic principles that are frequently embedded in social influence techniques (i.e., reciprocity, authority, and likeability), and are confronted with a persuasive request to either voluntarily donate time, effort, or actual money regarding different causes. Results show that being low in (state or trait) self-control capacity increases compliance with a request, provided that a salient decisional heuristic is present in the persuasion context.

Together, Chapters 2 and 3 demonstrate that the two stages that comprise the model that was introduced in the current chapter of this dissertation are linked by a single psychological process, that of active self-regulation, which relies on a limited resource. We also provide direct evidence for the linkage between Stage 1 and Stage 2, as Chapter 2 also includes a mediation analysis, showing that the initial request-phase of a social influence technique induces self-regulatory resource depletion, which subsequently affects susceptibility to compliance with the target request of the technique.

Chapter 4 extends the research presented in the first two empirical chapters, in focusing on the motivational aspects of self-control. The previous studies have demonstrated that dealing with an influence attempt involves controlled guidance by the self, and have shown that a state of self-regulatory resource depletion increases susceptibility to influence. These results suggest that successful resistance of a persuasive attempt will be dependent on the availability of resources to actively control the self. When resources are low, efficiently allocating the resources that remain seems to be a successful strategy for defense. However, one should be motivated to do so. Specifically, the research in Chapter 4 proposes that individuals low in self-control resources can still be successful at resisting a persuasive attack when prompted to be efficient in allocating their remaining self-regulatory resources. Three studies tested this hypothesis and showed 
that depleted individuals can be motivated to conserve their remaining resources when they expect to be confronted with an influence attempt in the nearby future. When they are warned about an influence attempt in advance, depleted participants temporarily sacrifice their self-control performance, which enables them to be just as successful at resisting the influence attempt as non-depleted participants. Results show that the effects are not attributable to receiving preliminary information about an upcoming encounter. Rather, it is the anticipation of an upcoming influence attempt that accounts for the effect. Throughout the experiments, different manipulations and measurements are used in order to secure the generalizability of the results. Resistance to persuasion is either measured as non-compliance with an (anticipated) persuasive request to do volunteer work, or more directly as the number of arguments generated against a persuasive request to donate money to charity. 



\section{Chapter 2}

Stage one:

\section{Weakening the ramparts:}

\section{Actively responding to an influence attempt induces self-regulatory resource depletion ${ }^{1}$}

${ }^{1}$ This chapter is adapted from two empirical articles:

Experiment 2.1 was previously published in Janssen, L., Fennis, B. M., Pruyn, A. Th. H., \& Vohs, K. D. (2008). The path of least resistance: Regulatory resource depletion and the effectiveness of social influence techniques. Journal of Business Research, 61(10), 1041-1045.

Experiments 2.2, 2.3, and 2.4 were previously published in Fennis, B. M., Janssen, L., \& Vohs, K. D. (2009). Acts of benevolence: A limited-resource account of compliance with charitable requests. Journal of Consumer Research, 35(6), 906-924. 

W

hy is saying "No" to fundraisers and sales representatives often so difficult, when they ask for money, or when they request you to sign a petition or buy their newest product? In many of these situations people are being targeted with a social influence technique, which is a clever persuasion attempt to increase the chance that consumers comply with a request. One characteristic that almost all successful social influence techniques have in common is that they consist of multiple requests. That is, the target request on which the influence agent hopes to gain compliance is preceded by one or several initial questions or requests. The intriguing fact that consumers are induced to comply with the target request at much higher rates when it is preceded by answering a series of initial questions, suggests that there is something special about the preliminary stage of social influence techniques that makes consumers especially willing to invest money, time, or effort, oftentimes without expecting a return on their investment. The present research provides support for the prediction that self-regulatory resource depletion (see Baumeister, Vohs, \& Tice, 2007) is an important factor in explaining the effectiveness of social influence techniques. More specifically, in the first chapter of this dissertation a two-stage model was introduced that provides a limited-resource account of the impact of sequential request techniques. This model states that a key reason why a scripted social influence tactic is so effective in generating compliance, is that actively responding to the initial request stage of such a technique requires controlled guidance by the self and induces a state of self-regulatory resource depletion. This weakened volitional state is then assumed to enhance compliance with a subsequent request, but only when the influence context contains heuristics aimed at promoting compliance (e.g., reciprocity), which nearly all scripted influence techniques naturally embed in the process (Cialdini, 1993). For a more general description of the theoretical background and a graphical depiction of the model we refer to Chapter 1 . In the present chapter we will elaborate the first stage of the model and provide a more detailed description of the accompanying premises. Moreover, we will present four empirical studies to support our predictions. 
In Stage 1 of our model we hypothesized that consciously attending and actively responding to one or multiple initial requests often involves deliberate, conscious, and controlled self-regulation, thus wearing down people's self-control resources. Indeed, meta-analytic comparisons spanning three decades of research on the most prominent sequential request procedure, the foot-in-the-door (FITD) technique, revealed that its effectiveness depends on specific attributes of the initial request to which people are exposed (Burger, 1999). Specifically, the FITD tactic is most effective when the initial request is highly involving. A closer look at FITD studies suggests that these highly involving initial requests entail either (a) demanding cognitive operations (b) active self-presentation, or both, processes that are known to elicit self-regulatory resource depletion (Schmeichel et al., 2003; Vohs et al., 2005).

In their first foot-in-the-door experiment, Freedman and Fraser (1966) approached households and before the larger target request was posed (i.e., a request to volunteer as a research participant in a large survey on household products), participants were asked whether they agreed to answer eight questions about the kinds of soaps they used. More important was that Freedman and Fraser (1966) also varied the extent of performance required with respect to the initial request. That is, participants either proceeded to actually answer the initial questions or only agreed to do so. The results showed that compliance with the target request was higher when participants had actually performed the initial request (53\%) rather than simply agreeing to do so (33\%). Furthermore, meta-analytic findings also align with our main argument that it is not the act of initial agreement per se that is the decisive factor in producing compliance but, rather, how much effort is required to accomplish the initial request (Burger, 1999).

Additionally, Fish and Kaplan (1974) asked participants to either listen to a lecture (low involvement) or craft and write an essay (high involvement) before the target request was posed. Seligman, Bush, and Kirsch (1976) asked for responses to five initial questions regarding 'people's reaction to the energy crisis' versus 20, 30, or 45 
questions (responding to more questions equaled higher involvement). In these studies, compliance with the target request (agreeing to complete an extensive survey) was higher when the initial request demanded more intellectual processing than when it was less intellectually demanding.

From a limited-self-regulatory resource perspective, these results make sense. Engaging in high-level intellectual processing (e.g., reading comprehension, crafting a logical argument) is known to tax self-regulatory resources (Schmeichel et al. 2003; Smit, Eling, \& Coenen, 2004). Hence, if the initial request phase of an influence technique is intellectually challenging, it likely leads to a more depleted state than if the initial request is less intellectually challenging. However, an initial request phase would be predicted to be relatively unsuccessful if it entailed answering only a few simple question(s). Rather, the key seems to be the extent to which the responses require effortful guidance by the self. In their work, Schmeichel et al. (2003) found that self-regulatory resource depletion impaired performance on cognitively demanding tasks but left performance on simpler mental tasks that use well-learned and standard procedures unaffected.

Next to manipulating involvement in terms of the extent to which the initial request required demanding cognitive operations, manipulations of high involvement in the initial request phase of a FITD technique often required effortful impression management. For example, Tybout (1978, Experiment 1) asked participants to simply sign a petition (low involvement) or asked them to explain to the influence agent their personal reasons for signing (high involvement), an act that likely induces selfpresentation motives. Pliner, Hart, Kohl, and Saari (1974) examined compliance with a request to donate money to the Cancer Society. The donation request was preceded either by asking participants to wear a daffodil pin or by asking them to wear the pin and persuade family members to wear the pin as well. Presumably, the act of persuading others to wear the pin engaged impression management processes since the target must present him/herself in a favorable and socially desirable light to family members 
regarding reasons to wear the pin. Compliance with the donation request was higher after people had agreed to approach family members to wear the pin than if they simply had been asked to wear the pin themselves. Recent work on the role of self-regulation has underscored the taxing nature of self-presentation processes (Vohs et al., 2005). This work demonstrated that active (but not habitual) forms of self-presentation lead to impaired self-regulation later due to depleted self-regulatory resources.

Finally, recent work on the disrupt-then-reframe (DTR) technique also implies that yielding to the opening stage of an influence attempt involves controlled selfregulation. In this tactic, an offer is presented to the target, followed by a subtle oddity or 'twist' in the sales script (such as stating the price of the offer in pennies before stating it in dollars), and finally a persuasive phrase that concludes the script (Fennis, et al. 2004, 2006; Kardes, Fennis, Hirt, Tormala, \& Bullington, 2007). Results from Fennis et al. (2004, Study 1) suggest that participants exposed to the DTR technique showed signs of self-regulatory resource depletion in that they were unable to generate as many counterarguments in response to the sales script as did participants who had not been exposed to the DTR technique (see Wheeler, et al. [2007] for counterargumentation as a process involving self-regulatory resources).

In sum, there is evidence in support of the hypothesis laid out in Stage 1 of our model: yielding to the initial request-phase of a multiple request influence procedure to gain compliance affects self-regulatory resource availability, because yielding involves either effortful self-presentation or intellectual demands. Stage 2 of the model, which will be more thoroughly discussed in Chapter 3 of this dissertation, proposes that this state of self-regulatory resource depletion drives the mindlessness so often observed in compliance contexts and thereby ups the odds that an individual will yield to the target request of an influence technique. 


\section{Outline of experiments}

Four experiments tested Stage 1 of our model, and showed that yielding to an initial request to answer a series of effortful self-presentational (Experiments 2.1, 2.2, and 2.3) or cognitively demanding questions (Experiment 2.4) induces self-regulatory resource depletion. In the first two field experiments we gathered evidence for our model by examining the initial request-phase of a typical foot-in-the door ploy aimed at fostering compliance. The findings supported the hypothesis that responding to a series of involving initial questions suffices to induce self-control failure. Additionally, in a follow-up field experiment (Experiment 2.3), we ruled out three potential alternative explanations that differences in duration between the conditions, emotion changes, or norm-violation by the influence agent could account for the effects on regulatory resource depletion. Finally, Experiment 2.4 links Stage 1 and Stage 2 of our model. By providing a formal test of mediation, it assesses whether self-regulatory resource depletion indeed functions as a pivotal intervening variable between the initial request phase and the target request phase in sequential request procedures that are aimed at promoting compliance.

\section{Experiment 2.1}

Experiment 2.1 provided a first test of our hypothesis that yielding to an initial request of a compliance-gaining procedure depletes self-regulatory resources. In this field study we presented people with a series of 11 open-ended questions about their health behavior and lifestyle. These questions are likely to heighten impression management motives, which is known to deplete participants' regulatory resources (Vohs et al., 2005). Participants in the no initial request condition were not asked the initial questions. We predicted an effect of responding to the initial request on self-regulatory resource depletion, which was measured by administering the State Ego Depletion Scale (Ciarocco, Twenge, Muraven, \& Tice, 2010). 


\section{Design and procedure}

The study employs a single factor (initial request vs. no initial request) between-subjects design. Sixty people (30 female, 30 male) voluntarily participated in this experiment. Their age varied from 18 to 73 years $(M=34.33, S D=16.28)$.

One of three confederates (one female, two male) randomly approached passers-by on a market square in the centre of a large town with a request to participate in a short study, being conducted by the health sciences department of the local university. The confederate asked participants whether they were willing to answer a few questions about their health behavior and lifestyle.

The confederate randomly assigned participants to the initial request or no initial request condition. In the initial request condition, the confederate presented participants with a series of 11 open-ended questions. These questions asked extensively about behaviors such as sports and exercising, smoking, use of alcohol, and eating habits. Examples of questions are "How much time do you monthly spend on sports and exercising?" and "Do you consciously pay attention to your eating habits?" These questions offered participants the opportunity to present themselves in a socially desirable manner by emphasizing the health-conscious nature of their lifestyle and eating habits. Participants in the no initial request condition did not receive any initial questions.

\section{Dependent measure}

Next, participants completed the State Ego Depletion Scale (Ciarocco et al., 2010) to measure self-regulatory resource depletion. Participants in the initial request-condition received a copy of this scale after answering the 11 open-ended questions, apparently as part of the inquiries about their health behavior. Participants in the no initial request condition received the scale immediately after the introduction of the confederate. On 
a 7-point Likert scale (1 = not true; 7 = very true) participants indicated their agreement with each of the 25 items of the State Ego Depletion Scale. Sample items include: 'Right now, it would take a lot of effort for me to concentrate on something,' 'I can't absorb any more information', and 'I feel sharp and focused' (reverse scored; see Appendix for a complete listing of the items). The average score on this scale served as a measure of self-regulatory resource depletion $(a=.90)$, with higher scores indicating more depletion. Finally, participants were debriefed, thanked, and dismissed.

\section{Results and discussion}

In line with our hypothesis, a t-test revealed a significant effect of the initial request on State Ego Depletion Scale scores $(t(58)=2.25, p<.05, d=.58)$. Participants who answered 11 open-ended questions about their health behavior and lifestyle scored higher on the State Ego Depletion Scale, and thus indicated that they were more depleted $(M=2.87$, $S D=1.00)$ than participants in the no initial request condition $(M=2.39, S D=.60)$.

This result of Experiment 2.1 provides initial support for Stage 1 of our model, the prediction that yielding to the initial request-phase of a sequential request technique negatively affects self-regulatory resources. Actively responding to multiple initial questions that are likely to heighten impression management motives appears to be a cognitive activity that requires self-control and depletes the self's energy resource.

\section{Experiment 2.2}

Experiment 2.2 sought to extend the results of Experiment 2.1 in two key ways. First, it is possible that the causal factor in producing depletion was not a request to answer a series of self-presentational questions but instead involved being confronted with an unanticipated conversation with an unknown person. In the current experiment, therefore, we contrasted an initial request condition in which participants had to 
answer a series of eight open-ended questions about personal health and lifestyle behaviors (presumably fostering active impression management motives, similar to Experiment 2.1) with a condition in which a confederate approached consumers on the street and asked them for directions to several town landmarks. In this condition (the 'landmark' condition), people were asked to point out three well-known locations on a map of the city in which the study took place. Second, to provide a stronger test of whether responding to an initial request would affect self-regulatory capacity, we assessed resource depletion with a validated self-control task: persistence in tracing an (unsolvable) figure (see Baumeister et al., 1998), instead of using a self-report scale as in Experiment 2.1. We predicted that answering the series of open-ended questions would diminish self-regulatory resources and thus reduce persistence on the subsequent figure-tracing task compared to the landmark condition.

\section{Design and procedure}

Forty-six people (20 female, 26 male; mean age 43.3 years, $S D=14.15$ ) volunteered to participate in a single factor (encounter: initial request vs. landmark) between-subjects design. One of two female confederates randomly approached passers-by in the centre of a large town, and randomly assigned them to one of the two conditions.

In the initial request condition, the confederate asked participants to answer a series of questions about their health behavior and lifestyle as part of a student project for the local university. The confederate then presented participants with a series of eight openended questions, similar to those in Experiment 2.1. In line with the results of Experiment 2.1 and earlier work by Vohs et al. (2005), these questions were thought to induce a state of self-regulatory resource depletion due to the impression management motives they aroused. In the landmark condition, the confederate approached participants while holding a map of the city and asked for directions by having participants point out three locations on the map: their current position, the town railwaystation and a main street. 


\section{Dependent measure}

To measure self-regulatory resource depletion, in both conditions participants were presented with a figure-tracing puzzle (Baumeister et al., 1998). To introduce this task, the confederate explained that she was also collecting data for a short study and was looking for participants. The confederate then gave instructions to trace a geometric figure (see Figure 2) without retracing any lines and without lifting the pencil from the paper. Multiple slips of paper were provided so that participants could make multiple attempts. They were told that they could take as much time and as many trials as they needed, and that they could stop whenever they wanted. Unbeknownst to participants, the task was impossible to solve. The amount of time participants worked on the unsolvable puzzle and the number of attempts they made were our measures of selfregulatory resource depletion (cf. Baumeister et al., 1998). When participants decided to stop, they were debriefed and thanked.

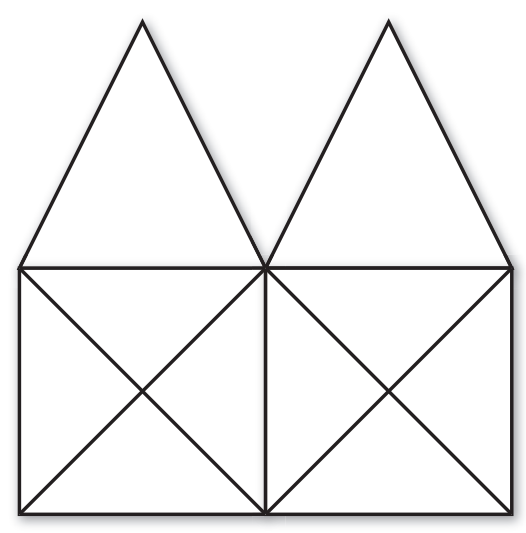

Figure 2. Geometric figure-tracing puzzle. 


\section{Results and discussion}

As predicted, simply having a several-minute interaction with an unacquainted person on the street did not explain the previously shown effects. Participants who answered eight open-ended questions about their health behavior and lifestyle worked on the unsolvable figure for a shorter amount of time $(M=62.04$ seconds, $S D=50.20)$ than did participants who conversed with a confederate about how to find several town landmarks $(M=141.62$ seconds, $S D=81.20), t(44)=-3.91, p<.001, d=1.18$. In addition to differences in temporal persistence, participants in the initial request condition put forth fewer attempts to solve the puzzle $(M=1.84, S D=.85)$ than did participants in the landmark condition $(M=4.67, S D=3.72), t(44)=3.41, p<.01, d=1.05$.

Together, these findings clarify and extend the results of Experiment 2.1. Answering questions that involve effortful self-presentation seems to be an important element in multiple request encounters in that it depletes self-regulatory resources. Moreover, the results of Experiment 2.2 indicate that the results of Experiment 2.1 cannot be attributed to the fact that participants were stopped in the street to have a conversation with a stranger.

\section{Experiment 2.3}

We performed another field study to exclude three other potential alternative explanations for the impact of an initial request on self-regulatory resource depletion. First, it is possible to interpret the finding of reduced self-regulatory resources among participants in the previous experiments as occurring because of differences in duration of the interaction (as a function of receiving an initial request vs. no initial request or indicating several town landmarks). Therefore, we homogenized the duration of both types of interactions and the time length of each interpersonal interaction was recorded and included in the analyses. Second, since (negative) affect may have played 
a role, we administered the PANAS (Watson, Clark, \& Tellegen, 1988), and included measures of liking of the influence agent. And finally, impaired persistence on the figure tracing puzzle in Experiment 2.2 is possibly attributable to a perception that the requesting agent 'overtaxed' the target or demanded too much of the target without a counter-concession of some sort, thus violating a norm of reciprocity (Gouldner, 1960). This perception may have increased resistance to accommodate the agent and the willingness to cut short the interaction, hence reducing performance on the figure tracing puzzle. To assess whether violating the norm of reciprocity accounted for the earlier findings, in Experiment 2.3 we varied the number of requesters (either one or two), and included a measure of feelings of resistance towards the requester. Following procedures outlined by Cialdini et al. (1975), if norm-violation by the agent in our previous experiment explained the earlier findings, we should expect increased participant resistance when the same confederate makes both the initial request and subsequent requests, but not when one confederate makes the first request and a second confederate makes the second request. Hence, only when the same confederate poses both requests the target could perceive this as receiving too many demands, resulting in less willingness to further accommodate the person.

In this field study we used the same conditions as in Experiment 2.2. We contrasted an initial request condition to answer a series of open-ended questions with a condition in which a confederate approached people in the street to ask for directions (landmark condition). Additionally, we included a measure of self-presentational effort, as to verify whether responding to the initial request generates more active impression management concerns than indicating a number of town landmarks. 


\section{Design and procedure}

Thirty-five people (14 female, 21 male; mean age 44.46 years, $S D=18.21$ ) participated in a 2 (encounter: initial request vs. landmark) X 2 (number of requesters: one vs. two) between-subjects factorial design.

Either one or two confederates ( 1 male, 1 female) randomly approached passers-by in the centre of a large town. Participants were randomly assigned to the initial request or landmark condition. Similar to Experiment 2.2, the confederate in the initial request condition asked participants to answer eight open-ended questions about their health behavior and lifestyle as part of a student project for the local university. In the landmark condition, the confederate approached participants with a map of the city and asked for directions. Next, based on a random schedule, either the same or a second confederate told participants that there was a second purpose of his/her activities that day, which was to study people's responses when having an interaction on the street. The confederate then asked participants to complete a questionnaire containing the dependent measures. After having completed the questionnaire, participants were thanked for their participation.

\section{Dependent measures}

Duration. Answering the series of questions in the initial request condition as well as giving directions in the landmark condition lasted an average of 2.5 minutes $(S D=44.42$ seconds), with no difference in duration, $t<1$.

Affect. To measure whether the type of encounter would result in unintended (negative) affect, we administered the Positive and Negative Affect Schedule (PANAS; Watson et al., 1988), which includes 10 positive and 10 negative affect-items rated on 5-point scales ( $1=$ not at all feeling this way; $5=$ extremely feeling this way). Sample items include: 
'irritable' (negative affect) and 'enthusiastic' (positive affect). An index of positive and negative affect was generated by averaging the scores on the respective items $(a=.83$ for the positive affect index and $a=.85$ for the negative affect index).

Liking. Liking for the confederate (that initiated the interaction) was measured on a 7-point scale ( 1 = not at all; 7 = very much), using ratings of the confederate on the adjectives 'friendly', 'sympathetic', and 'enjoyable to talk with'. There was sufficient reliability to combine them into one index $(a=.75)$.

Resistance. Feelings of norm-violation when encountered by the confederate were measured with an index averaging the scores on five items (measured on a 7-point scale, $1=$ not at all; $7=$ very much, $a=.85$ ) that asked participants to rate the extent to which participants felt resistance and reluctance to interact, unwillingness to engage in the conversation, the urge to walk on, and regret about the interaction.

Self-presentational effort. Finally, we assessed the extent to which the encounter (either responding to the initial request or giving directions) involved effortful self-presentation. With an index of two items rated on a 7-point scale ( $1=$ not at all; $7=$ very much, $r=.33$, $p<.05)$ we measured the extent to which participants exerted considerable effort while interacting and were motivated to make a very positive impression on the confederate

\section{Results and discussion}

We used a series of (multivariate) analyses of covariance to test our hypotheses. The covariate (duration of interaction) was not a significant predictor of any of the dependent measures and is not discussed further. As expected, mood states were not different as a function of condition: A MANCOVA with type of encounter (initial request vs. landmark) and number of requesters (one vs. two) as independent variables and positive and negative affect as dependent variables showed no significant differences 
across conditions. That is, neither the main effect of type of encounter $(F<1)$, nor the main effect of number of requesters $(F(2,29)=2.51, n s)$, nor their interaction $(F(2$, $29)=1.41, n s)$ was significant. Hence, differences in mood were likely not an issue in Experiment 2.2.

An ANCOVA with the same independent variables and liking for the confederate as dependent variable also failed to produce significant effects. Hence, differential liking for the confederate likely did not drive the earlier results, since there was no main effect of type of encounter $(F<1)$, no main effect of the number of requesters $(F<1)$, and no interaction effect $(F<1)$. Moreover, we can rule out increased resistance as a function of norm-violation by the requester since the ANCOVA with type of request and number of requesters did not result in the required interaction effect, $F(1,30)=1.11$, ns. Both main effects also failed to reach significance (both $F s<1$ ).

In support of our underlying premise, the only significant effect was a main effect of the type of encounter on participants' reports of active self-presentation, $F(1,30)=4.73, p<$ $.05, d=.68$. Inspection of the means showed that answering a series of personal health and lifestyle-related questions required more effortful self-presentation $(M=3.47, S D$ $=1.02$ ) than simply having an unanticipated conversation with a confederate about several town landmarks $(M=2.74, S D=1.11)$. As expected, there was no main effect of the number of requesters $(F<1)$, nor an interaction effect between the number of requesters and the type of encounter $(F<1)$.

In short, these results suggest that the type of encounter influenced active impression management concerns but did not affect emotions, liking for the confederate, or perceptions of receiving too many demands from the requester. Moreover, the finding of reduced self-regulatory resources among participants in the previous experiment is not likely to be attributable to differences in time length of the interpersonal interactions in the initial request condition and the landmark condition. 


\section{Experiment 2.4}

The previous studies demonstrated the pivotal role of self-regulatory resource depletion as an outcome of responding to the initial stage of a social influence technique designed to promote compliance. Yet the two stage model posits that regulatory resource depletion functions as a mediating variable, produced by the initial stage of a compliance gaining technique and, in turn, fostering compliance with the target request (when heuristics in the influence context promote compliance). The present experiment, which was conducted in a laboratory setting instead of a field setting, sought to bridge Stage 1 and Stage 2 of the model by directly assessing this mediating role.

In line with Experiments 2.2 and 2.3, the present experiment contrasted a highly involving initial request condition with a condition in which a confederate approached participants with an equal number of questions of a less demanding nature. To balance the scope and domains of the findings thus far, we varied another key factor of successful sequential request techniques: the extent to which responding to the initial request requires high levels of cognitive effort (see Burger, 1999), instead of self-presentational questions as used in the previous experiments. A full foot-in-the-door (FITD) procedure was employed, consisting of continuing questions (see Burger, 1999). In line with a traditional continuing questions procedure, the initial questions foster compliance by being conceptually related to the target request. Compliance is triggered by people's desire to behave congruently across situations, functioning as a salient decisional heuristic. In the current experiment the initial questions dealt with knowledge regarding consumer taxes and the target request that was posed entailed participants' willingness to serve as a future research participant for the Dutch Tax and Customs Administration. Moreover, the current procedure ascertained that duration and extent of dialogue of both conditions were equivalent and therefore could not act as design confounds in the present experiment. 
In extension to Experiments 2.2 and 2.3, we kept consistent the topic of the questions between the highly demanding FITD condition and the less demanding condition. Before we exposed participants to a target request eliciting compliance, we measured selfregulation using the well-known Stroop task (Stroop, 1935), which has been shown to be a valid way to measure self-control ability (Vohs et al., 2005; Wallace \& Baumeister, 2002). Our prediction was that the type of influence technique would evince a main effect on selfregulatory resource depletion. Moreover, we expected that self-regulatory resources would mediate the impact of the type of technique on compliance, as the two-stage model holds.

\section{Design and procedure}

A total of 37 undergraduate students (10 female, 27 male, mean age 21.3 years, SD = 2.30) participated in a single factor (type of FITD influence attempt: demanding vs. undemanding initial request) between-subjects design.

Prior to arrival at the laboratory, participants were randomly assigned to condition. Upon arrival, participants were told that they would take part in a study on consumer behavior ostensibly conducted on behalf of the Dutch Tax and Customs Administration and were to be paid $€ 2.50$ for participating. In the cognitively demanding FITD condition, participants were presented with an initial request, which asked them a series of 10 challenging questions regarding their knowledge of consumer taxes and the Tax and Customs Administration. Answering these questions required high-level intellectual processing. In the undemanding condition, the 10 questions were on the same topic (consumer taxes) but did not require active and controlled problem solving and therefore were considered less demanding (see Schmeichel et al., 2003). For instance, in the cognitively demanding FITD condition, participants were asked: "One of the problems of the Tax and Customs Administration is the fact that many people tend to submit their tax claims after the yearly deadline. What steps can the Administration take to prevent this without giving citizens a fine?" In contrast, one of the questions in 
the undemanding condition was: "What is the first thing that comes to mind when you think about the Tax and Customs Administration?"

\section{Dependent measures}

Cognitive effort. We assessed the extent to which responding to the initial request involved differential cognitive effort using 10-point Likert scales ( 1 = not at all; $10=$ very much). To this end, we administered two items assessing the extent to which the participant reported (a) having exerted considerable mental effort while responding to the initial request, and (b) thinking extensively before answering the questions.

Self-regulatory resource depletion. We used the Stroop task (Stroop, 1935) to assess selfregulatory resource depletion. Several studies have established that performance on the Stroop task taxes self-regulatory resources (Gailliot, Baumeister et al., 2007; Gailliot, Plant, Butz, \& Baumeister, 2007; Muraven et al., 2006; Wallace \& Baumeister 2002; Webb \& Sheeran, 2003). For participants low in regulatory resources it is harder to inhibit or override the automatic habitual inclination to respond to the semantic meaning of the word and instead report the font color in which the word is printed. Hence, participants who are more depleted of their regulatory resources should take longer to report the ink color than less depleted participants (Webb \& Sheeran, 2003). Participants responded to each stimulus by clicking one of four buttons on their computer screen, which corresponded to the various color words. Participants received 32 randomized trials, of which 8 were congruent (a stimulus word was presented in a font color that matched its semantic meaning; e.g., 'blue' was presented in blue font) and 24 were incongruent (a stimulus word was presented in a font color that mismatched its semantic meaning; e.g., 'blue' presented in red font). Participants were instructed to report the font color of each word as quickly as possible, and the time it took participants to respond to each trial was recorded. In line with previous research (e.g., Muraven et al., 2006; Webb \& Sheeran, 2003), average reaction times on the Stroop task served as our main dependent variable of self-regulatory resource depletion. 
Compliance. In all conditions, the target request was whether participants would be willing to volunteer as a future research participant for studies conducted on behalf of the Dutch Tax and Customs Administration. Compliance was measured by the number of studies for which participants volunteered (possible range $0-10$ ).

\section{Results and discussion}

The present experiment assessed the impact of a sequential request technique that used an initial request that was cognitively demanding as opposed to one that was relatively undemanding. Our first test therefore assessed participants' reports of exerted mental effort while responding to the initial request. In support of our assumptions, we found an effect of type of request on reports of exerted mental effort $(t(35)=3.19, p<.01, d$ $=1.06$ ) and on the extent to which participants reported having thought extensively before answering the questions $(t(35)=3.58, p<.001, d=1.18)$. The means showed that responding to an initial request that was comprised of a series of cognitively demanding questions required more mental effort $(M=7.39, S D=1.65)$ and induced a stronger need to think extensively $(M=7.28, S D=1.99)$ than responding to a similar number of less taxing questions on the same subject $(M=5.32, S D=2.24$ and $M=4.89, S D=2.05$, respectively).

Mediation Analysis. The key objective of the present study was to assess whether self-regulatory resource depletion mediates the impact of a sequential request social influence technique on compliance with a volunteering request. To this end, a mediation analysis was performed following suggestions by Baron and Kenny (1986). These authors claim that demonstrating mediation requires estimating a series of regression models that first regress the mediator on the independent variable; then, second, regress the dependent variable on the independent variable; then, third, regress the dependent variable both on the independent variable and on the mediator. Full mediation is demonstrated when the independent variable significantly affects the 
mediator in equation 1 , the independent variable significantly affects the dependent variable in equation 2 , and the mediator significantly affects the dependent variable in equation 3 while the impact of the independent variable is rendered nonsignificant. The results of these analyses supported our predictions. First, the type of influence attempt (dummy coded) significantly predicted self-regulatory resource depletion as indexed by performance on the Stroop task $(\beta=.42, t=2.72, p<.01)$. The means showed that participants who responded to an initial request that comprised cognitively demanding questions had slower reactions on the Stroop task $(M=1.46 \mathrm{sec}, S D=.32)$ than participants who responded to an initial request that comprised undemanding questions $(M=1.24 \mathrm{sec}, S D=.24)$. As the second step, type of influence attempt significantly affected compliance rates $(\beta=.34, t=2.11, p<.05)$. In line with predictions, participants exposed to a sequential request technique that included a cognitively demanding initial request were more willing to act as a future research participant $(M$ $=2.28, S D=2.45$ ) than were participants in the undemanding initial request condition $(M=.95, S D=1.22)$. As the third step, the regression analysis with type of influence attempt and self-regulatory resource depletion (i.e., Stroop performance, centered) as predictors and compliance as the criterion showed that self-regulatory resource depletion significantly predicted compliance rates $(\beta=.40, t=2.42, p<.05)$, whereas the effect of type of influence attempt on compliance was reduced to nonsignificance $(\beta=.17, t=1.03, n s)$.

In sum, these results provided support for our two-stage model, not only by providing converging evidence for the first part of the model that sequential request techniques elicit self-regulatory resource depletion, but additionally showing that this state of reduced self-control in turn promotes yielding to a target request. Please note that compliance as the product of self-regulatory resource depletion occurs because the sequential request technique has embedded in it a heuristic principle. In this experiment, we employed a FITD ploy consisting of continuing questions (see Burger, 1999). The heuristic principle pointing to compliance in this case was the principle of consistency 
- that is, the propensity to behave congruently across situations. The next chapter of this dissertation will address in detail the role of self-regulatory resource depletion in responding to persuasive heuristics.

\section{General discussion}

Four studies tested the first stage of our two-stage model to account for the role of consumer self-regulatory resource depletion in the effectiveness of sequential request scripts that are used by professional fundraisers and sales representatives to elicit compliance. Stage 1 of the model posits that responding to an involving initial request (be that answering a series of cognitively demanding questions or questions that prompt self-presentational responses) reduces the supply of self-regulatory resources within the target. We found that responding to a series of questions (the typical first step in most multiple request strategies, see Burger, 1999; Cialdini \& Goldstein, 2004) induced a state of self-regulatory resource depletion. Moreover, the effects of yielding to this initial request were found both in the lab (Experiment 2.4) as well as in a naturalistic setting outside the laboratory using actual consumers as participants (Experiments 2.1, 2.2, and 2.3). The effects were witnessed on the extent of self-reported levels of resource depletion (Experiment 2.1), persistence on an unsolvable puzzle (Experiment 2.2, cf. Baumeister et al., 1998), as well as suppressing initial responses on a Stroop task (Experiment 2.4, cf. Webb \& Sheeran, 2003).

Converging evidence was found in two other studies (Fennis et al., 2009, Experiments 1 and 2), one administered in the lab and one in a fieldsetting, which presented participants with a highly involving initial request, either involving active self-presentation or demanding cognitive operations. As compared to participants not exposed to an initial request, these participants showed an impairment in counterargument generation (cf. Wheeler et al., 2007) and reduced performance on complex reasoning as a measure of intellectual functioning (cf. Schmeichel et al., 2003). Taken together, the effect sizes 
obtained in a total number of six lab and field studies are of comparable magnitude, suggesting that none of the presented results were unduly influenced by settingspecific biases, such as self-selection, demand characteristics, or differential attrition.

Based on earlier studies (see Burger, 1999), it was predicted that the effect of responding to an initial request on regulatory resource availability would be particularly salient when responses to the initial request required cognitively demanding answers or effortful self-presentation, both of which are known elicitors of self-regulatory resource depletion (Schmeichel et al., 2003; Vohs et al., 2005). This notion was put to the test directly in the four experiments presented in this chapter and additionally in Experiments 1 and 2 from Fennis et al. (2009), where people had to answer a series of involving questions that were either self-presentational ( 8 or 11 questions about personal health and lifestyle, 20 questions on personal eating habits) or cognitively demanding (3 questions regarding knowledge about $\mathrm{CO}_{2}$ emissions, 10 questions regarding knowledge on consumer taxes). The results allowed us to rule out several alternative explanations, such as the potential confounding role of negative emotions, violations of the norm of reciprocity, the duration of the interaction between agent and target, or the simple act of an unanticipated conversation with an unacquainted person.

One might wonder to what extent our studies reflect the 'classic' manipulations of compliance-gaining used by Freedman and Fraser (1966) and others (e.g., Pliner et al., 1974) in which participants were asked to agree with a small request before the larger target request was posed. At first glance, this procedure may appear at odds with our work in which we focused on the extent to which initial agreement involved effortful responding. Yet at closer inspection, the procedures used in the seminal Freedman and Fraser (1966) study bear a striking resemblance to our compliance-gaining scripts (e.g., answering eight questions about the kinds of soaps participants used). Moreover, a key driver in that research also proved to be the extent of performance of the initial request, rather than agreement per se. 
In the current work we used a foot-in-the-door procedure, the most prominent example of a variety of compliance-gaining techniques that have been reported in the literature. However, other compliance-gaining techniques are expected to be effective via the same mechanism as that which we revealed (i.e., self-regulatory resource depletion), to the extent that the opening stage of the technique requires effortful self-regulation. We await further research to confirm this expectation. In the final chapter of this dissertation, the boundary conditions to the present two-stage model will be discussed in more detail.

The present research also provided essential evidence that self-regulatory resource depletion indeed functions as a mediating variable between the response to the initial request and the response to the target request in sequential request techniques. This mediation demonstrated that the two stages that comprise the model presented in this dissertation are indeed linked by a single psychological process, that of active selfregulation. In this respect, the present research is the first to provide a self-regulatory resource depletion account of the effectiveness of social influence techniques. Another test of mediation was provided by a field study (Fennis \& Janssen, under review) which similarly demonstrated that regulatory resources mediate the impact of a foot-in-thedoor technique on compliance, and once more ruled out (negative and positive) mood as an alternate explanation, as well as a general tendency for acquiescence in sequential request settings.

The present research aimed to shed light on the process by which consumers are induced to invest money, time, or effort, failing to say "No" to fundraisers, sales representatives and other influence agents. Our research reveals that one key feature of effective influence tactics is the wearing down of self-regulatory resources that would otherwise be put toward resistance. In the next chapter we moved to testing the second stage of our model. We predicted that reduced self-control capacity would foster reliance on heuristics in decision-making, thereby increasing the chances of compliance. 




\section{Chapter 3}

Stage two:

The path of least resistance:

Self-regulatory resource depletion affects compliance through heuristic decision making ${ }^{2}$

${ }^{2}$ This chapter is adapted from two empirical articles:

Experiments 3.1 and 3.3 were previously published in Fennis, B. M., Janssen, L., \& Vohs, K. D. (2009). Acts of benevolence: A limited-resource account of compliance with charitable requests. Journal of Consumer Research, 35(6), 906-924.

Experiment 3.2 was previously published in Janssen, L., Fennis, B. M., Pruyn, A. Th. H., \& Vohs, K. D. (2008). The path of least resistance: Regulatory resource depletion and the effectiveness of social influence techniques. Journal of Business Research, 61(10), 1041-1045.

Experiment 3.4 was not previously published. 

$I$ n the previous chapter, four studies were presented that tested the first stage of a two-stage model, introduced in Chapter 1, which represents a self-regulatory resource depletion account of the impact of sequential request techniques. This model states that a key reason why a scripted social influence tactic is effective in generating compliance, is that actively responding to the initial request stage of such a technique requires controlled guidance by the self and induces a state of self-regulatory resource depletion. This weakened volitional state then is assumed to enhance compliance with a subsequent request, but only when the request contains heuristics aimed at promoting compliance (e.g., reciprocity), which nearly all scripted influence techniques naturally embed in the process. For a more general description of the theoretical background and a graphical depiction of the model we refer to Chapter 1. The results of extensive empirical testing of the first stage of this model were provided in Chapter 2. In the present chapter we will elaborate the second stage of the model and provide a more detailed description of the accompanying premises. Moreover, we will present four empirical studies that support our predictions.

Recently, research has begun to test the link between self-regulation failure and persuasion. This work suggests that a state of self-regulatory resource depletion weakens resistance to temptations and (unwanted) influence attempts (Baumeister, 2002). For example, Burkley (2008) showed that resistance to persuasion attempts requires active self-control and therefore depletes regulatory resource stores, particularly when the persuasive message is highly involving.

Another test of the impact of self-regulatory resource depletion on resistance to persuasion was presented by Wheeler et al. (2007). In their study, participants were asked to resist a counterattitudinal persuasive message. Participants whose selfregulatory resources had been depleted by a previous and unrelated self-regulation task showed less resistance than non-depleted participants, especially when message arguments were weak. Similar to the findings by Fennis et al. (2004, Experiment 1), these 
participants generated fewer counterarguments in response to the persuasive message than did non-depleted participants. In line with dual-process frameworks (Chaiken \& Trope, 1999), the key seemed to be that self-regulatory resource depletion hindered the processing of message-relevant information as evidenced by reduced sensitivity to argument quality.

Extending the dual-process logic, Wheeler et al.'s findings (2007) point to heuristic processing as a consequence of self-regulation failure. That is, if self-regulatory resource depletion reduces systematic or central-route processing, then it should enhance the weight on heuristic processing in consumer judgment and decision making. The notion that a state of mindlessness drives the employment of heuristics in decision making is well established in various domains, such as persuasion (e.g., Petty \& Wegener, 1999), judgment and choice (Ferreira, Garcia-Marques, Sherman, \& Sherman, 2006), and compliance (Cialdini, 1993). The heuristic-systematic processing model of persuasion (Chaiken, 1980) states that under conditions of mindlessness, recipients of persuasive messages typically resort to simple heuristics to arrive at a judgment. In a seminal study, Chaiken (1980) showed that mindless (low involvement) recipients used a simple source-related heuristic (e.g., 'likeable sources can be trusted') in evaluating a message, whereas mindful (high involvement) participants depended on argument quality. Important for the present research, mindless message recipients were not susceptible to influence by the persuasive message by default, but only to the extent that a suitable heuristic was present in the persuasion context.

The idea that reliance on heuristics leads to compliance has been inferred but not formalized empirically. Many compliance-gaining techniques are assumed to be effective under mindless conditions because they trigger a fixed action pattern (to wit, Cialdini's famous click, whirr effect, 1993), which encourages acquiescence to the request. As a classic example, Langer et al. (1978) showed that people are more willing to yield to a request when the requester provides a reason for doing so. Significantly 
more participants waiting in line to make photocopies accommodated a confederate barging ahead of the line if he or she gave a reason, regardless of whether the reason was legitimate ("Because I'm in a rush") or trivial ("Because I have to make some copies"). These results underscore our idea that a suitable and compliance-promoting heuristic (in the Langer et al. case, the 'because heuristic') must be present in the influence context in order for a state of mindlessness to result in compliant behavior. Please note that we do not argue that such heuristic decision making results in compliance per se, but only to the extent that the heuristic points to compliance as an efficient behavioral outcome.

In sum, our approach is the first to offer an in-depth account for a single underlying process explaining why and how mindlessness may result in compliance with persuasive requests across many social influence techniques typically employed by influence professionals. As presented in the introductory chapter of this dissertation, our key postulate is a two-stage model that accounts for the influence of sequential request techniques on compliance. Recall that these two stages are: (1) the initial request phase of sequential request techniques induces mindlessness through a state of self-regulatory resource depletion, and (2) depletion-induced mindlessness heightens compliance through reliance on heuristics. This compliance manifests itself in greater willingness and actual performance of acts of compliance such as freely donating (sometimes substantial) amounts of time, effort, or money without necessarily expecting something in return. 


\section{Outline of experiments}

Four experiments tested Stage 2 of our model. In this series of lab and field studies, we demonstrated that self-regulatory resource depletion fosters the use of heuristics in decision-making, thereby increasing the chances of compliance with a request. In Experiment 3.1, the availability of self-regulatory resources was manipulated, as was the salience of a heuristic principle (in this case, the principle of reciprocity that is featured in the door-in-the-face technique, Cialdini, 1993; Cialdini et al., 1975; Gouldner, 1960). Subsequently, compliance with a request to voluntarily participate in future research was measured. We predicted and found that when participants were in a state of selfregulatory resource depletion and were presented with a heuristic for reciprocity, they showed a clear tendency to comply with the request. We extended the generalizability of our theorizing in Experiments 3.2 to 3.4 by including other operationalizations of selfregulatory resource depletion. We also demonstrated the effect in both field and lab settings involving different heuristics that are frequently embedded in social influence techniques (the principles of authority in Experiment 3.2 and likeability in Experiment 3.3, Cialdini, 1993), and different acts of compliance. Moreover, whereas Experiments 3.1 to 3.3 involve situational manipulations of reduced self-control, Experiment 3.4 obtained converging evidence by investigating the role of dispositional self-control.

\section{Experiment 3.1}

In Experiment 3.1 we induced a state of self-regulatory resource depletion with a self-control task and manipulated the salience of the heuristic principle of reciprocity (Gouldner, 1960). Next, participants were presented with a target request to volunteer as a research assistant, with amount of time participants were willing to volunteer as our compliance measure. We expected self-regulatory resource depleted participants to show increased compliance with the target request, compared to their nondepleted counterparts, but only when the reciprocity principle was made salient. 


\section{Design and procedure}

One hundred and eight students enrolled in various undergraduate programs (71 female, 37 male; mean age 20.51 years, $S D=2.02$ ) participated in exchange for partial course credit. The study used a 2 (self-regulatory resource depletion condition: depletion vs. no depletion) X 2 (heuristic-activation: reciprocity vs. no reciprocity) between-subjects factorial design.

Prior to arrival at the laboratory, participants were randomly assigned to one of the four conditions. Participants were told that the experiment consisted of several unrelated tasks. We induced self-regulatory resource depletion with a self-control task adopted from Baumeister et al. (1998). All participants were given typewritten sheets of paper with dense text (from a highly advanced statistics book) and were instructed to cross off all instances of the letter e. After completing one sheet, participants in the no depletion condition were instructed to continue with the same task on a different sheet of typewritten paper. Participants in the depletion condition, in contrast, were then told to learn and apply new rules about when and whether to cross off occasions of the letter e. Compared to participants in the no depletion condition, participants in the depletion condition had to engage in more self-control to inhibit the overlearned response of crossing out every $e$ and instead use more complicated and cognitively demanding rules. Previous research has shown that these two conditions produce significant differences in the supply of self-regulatory resources (Baumeister et al., 1998).

The next step involved manipulating the salience of the reciprocity heuristic. In the reciprocity condition, the experimenter told participants that she would make an exception and excuse them from the next part of the experiment, because she decided she had collected enough data on the test. She told that this part of the experiment would have involved a mathematical test that other participants thought was quite dull and boring. Participants in the no reciprocity condition were not told about a math test or anyone being excused from it. The concession made by the experimenter was aimed at inducing a counterconcession on the part of the participant (Cialdini, 1993) in the form of increased compliance with the volunteering request. 
Dependent measure

Next, the experimenter instructed participants to sit at a desk with a computer. After clicking a button, the following message appeared on the screen: 'For next year, researchers of the Department of Communication are looking for students who are willing to voluntarily participate as an experimenter during research. If we'd ask you this favor, how much time would you be willing to participate?' Participants could answer this target request on a scale ranging from 0 to 240 minutes in 30-minute intervals. Length of time participants were prepared to volunteer was our measure of compliance (cf. Kardes et al., 2007). Afterwards, participants were debriefed and thanked.

\section{Results and discussion}

Overall, 70\% of the participants agreed to act as a volunteer in response to the target request. An ANOVA was conducted on degree of compliance with the target request as a function of depletion condition (depletion vs. no depletion) and heuristic-activation (reciprocity vs. no reciprocity). This analysis showed that the interaction between depletion condition and heuristic activation condition was significant $(F(1,104)=$ $\left.10.22, p<.01, \eta^{2}=.09\right)$. As predicted, analysis of the simple main effects showed that the effect of resource depletion on compliance was only significant in the reciprocity condition $(F(1,104)=19.36, p<.001, d=1.16)$. After being given the reciprocity cue, participants who had completed the rule-switching version of the crossing out $e$ task (i.e., who were depleted of their regulatory resources) showed higher compliance by volunteering more time $(M=96.00 \mathrm{~min}, S D=52.05)$ than non-depleted participants $(M$ $=41.61 \mathrm{~min}, S D=41.48)$. When the reciprocity principle was not made salient, however, availability of self-regulatory resources had no effect on compliance rates $\left(M_{\text {depletion condition }}\right.$ $=43.45, S D=43.61$ versus $M_{\text {no depletion condition }}=42.86, S D=36.90, F<1$, see Figure 3.1). 


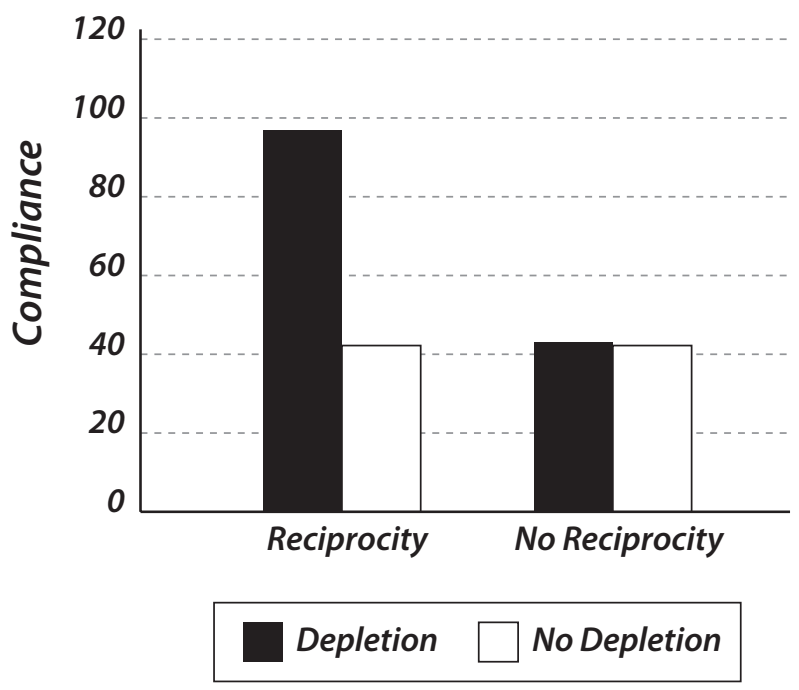

Figure 3.1. Average number of minutes participants volunteered to serve as an experimenter as a function of depletion-induction and heuristicactivation (adapted from Fennis et al., 2009).

The statistical model also showed a main effect of both factors. Participants who were depleted of their regulatory resources were overall willing to spend more minutes voluntarily participating as an experimenter $(M=64.90, S D=53.51)$ as compared to participants in the no depletion condition $(M=42.20, S D=39.04), F(1,104)=10.68, p<$ $.01, d=.48$. Participants also complied more with the request when the heuristic principle of reciprocity was made salient $(M=62.94, S D=52.74)$, compared to compliance rates in the no reciprocity condition $(M=43.16, S D=40.10), F(1,104)=9.30, p<.01, d=.42$.

The results of Experiment 3.1 support Stage 2 of our model showing that self-regulatory resource depletion fosters compliance with a request through reliance on heuristics. We observed greater compliance with the request when self-regulatory resources had been lowered as compared to when they had been untouched, but only when a compliancepromoting heuristic was part of the influence setting. Notice that in the no reciprocity condition, self-regulatory resource depletion per se did not result in enhanced compliance, suggesting that even people in a weakened state can see through blunt, 
direct attempts at being influenced (Friestad \& Wright, 1994). Rather, depletion appears to increase susceptibility to influence attempts only when the influence attempt contains the lure of a suitable heuristic that can function as the basis for decision making. To generalize these findings beyond the specific type of self-regulatory resource depletion induction, heuristic principle, and type of compliance in Experiment 3.1, the following two studies varied each of these variables.

\section{Experiment 3.2}

The purposes of Experiment 3.2 were to replicate the results of Experiment 3.1, using a different manipulation of self-regulatory resource depletion and activating a different heuristic principle. Additionally, this study extends the results of the previous study by focusing on another form of compliance: actual money donated to a charitable cause. We induced self-regulatory resource depletion with a self-control task adopted from Schmeichel et al. (2003). Participants were subsequently presented with a request to donate money to a charity organisation, which either was or was not described as a source of high authority, to activate this heuristic principle. We predicted that selfregulatory resource depleted participants would donate a larger percentage of the money that they received for participating in the experiment to charity (cf. Vohs, Mead, \& Goode, 2006), but only when this charity was described as a source of high authority.

\section{Design and procedure}

The study employs a 2 (self-regulatory resource depletion condition: depletion vs. no depletion) $\times 2$ (heuristic-activation: authority vs. no authority) betweensubjects factorial design. A total of 107 undergraduate students (70 female, 37 male) served as participants in this study, either in exchange for 6 euro's or in exchange for 2 euro's and course credit. Their mean age was 20.76 years $(S D=2.15)$. Upon arrival at the laboratory, the female experimenter randomly assigned participants to 
one of the four conditions. She told participants that the experiment concerned nonverbal assessments of personality.

A state of resource depletion was induced with an attention control video adopted from Schmeichel et al. (2003). Participants were asked to watch a short videotape. This 4-minute videotape (without audio) featured a woman being interviewed by an off-camera interviewer. According to the instructions, participants would later be judging the women's personality based on her non-verbal behavior. In addition to the woman being interviewed, the tape showed a series of common one-syllable words (e.g., 'hat') at the bottom quarter of the screen for 10 seconds each. These words were not related to the woman being interviewed. Participants in the no depletion control condition received no instructions regarding the irrelevant words, nor were they made aware of the words prior to viewing the video. Participants in the depletion condition read the instruction 'not to read or look at any words that may appear on the screen' and 'to redirect their gaze to the woman if they found themselves looking at the words'. Previous research has shown that regulating attention this way is effortful and depletes regulatory resources (Schmeichel et al., 2003; Vohs \& Faber, 2007).

After watching the videotape participants read a short message on their computer screen, asking them to consider donating (part of) their participant-money to a charity organisation. The heuristic principle of authority either was or was not activated by introducing either a well-known organisation (The Red Cross), which was described as renowned and experienced, or a relatively unknown organisation (Association for African Projects), described as having starting experience in relief work. The domain of charity was the same in both conditions and concerned the development of educational projects in Third World countries. The charity organisation that was presented as an authority would presumably invoke more compliance, since research shows that people are more willing to comply with requests of authority figures, or-more generally- sources of high authority and credibility, either persons or institutions (see Cialdini, 1993). 
Dependent measure

After reading the description of the charity organisation, participants could indicate the amount of money they were willing to donate. Afterwards this amount was subtracted from the amount of money participants would receive for their participation in the experiment and they were paid the difference. The percentage of money that participants actually donated served as our measure of compliance. All participants were debriefed and thanked. The total amount of money donated during this experiment was transferred to the two charity organisations.

\section{Results and discussion}

A fairly high percentage of participants (80\%) complied with the request to donate money to charity. An analysis of variance on the percentage of money donated, with self-regulatory resource depletion condition (depletion vs. no depletion) and heuristicactivation (authority vs. no authority) as independent variables showed a main effect of depletion as well as an interaction-effect. Participants who were depleted of their regulatory resources by the attention control video were willing to donate a larger percentage of their money $(M=.73, S D=.38)$ than participants in the no depletion control condition, who did not have to control their attention during the video $(M=.57$, $S D=.43), F(1,103)=5.31, p<.05, d=.39$.

Of main interest for our hypothesis is the finding that the interaction between selfregulatory resource depletion and heuristic-activation proved to be significant $(F(1,103)=4.46, p<.05)$. Analysis of the simple main effects shows that the effect of resource depletion on compliance was only significant when the authority principle was activated $(F(1,103)=8.69, p<.01, d=.94)$. In these conditions, resource depleted participants donated a larger percentage of their money $(M=.81, S D=.32)$ than non-depleted participants $(M=.46, S D=.42)$. When the authority-principle was not 
activated, depletion did not affect compliance rates: the difference in percentage of money donated between participants in the depletion condition $(M=.66, S D=.42)$ and no depletion condition ( $M=.64, S D=.43$ ) was not significant $(F<1$, see Figure 3.2).

Note that the presence of the heuristic principle of authority was manipulated by introducing a renowned and experienced organisation that participants could donate money to, as compared to a relatively unknown organisation with starting experience in the no authority control condition. Participants appear to be only susceptible to the authority heuristic when they are depleted of their regulatory resources, donating a larger percentage of their money as compared to the no depletion control condition. This result parallels the findings of Fennis et al. (2006), who showed that a disruptthen-reframe social influence procedure (DTR, for a more detailed description of the technique see Chapter 2) generated more compliance when it incorporated a familiar brand, as compared to an unfamiliar brand. Participants were asked to purchase (multiple sets of) playing cards from a salesperson that either introduced herself as a representative of Amstel, a well-known Dutch brand of beer, or as a representative of the fictitious (and therefore unfamiliar) brand of soft drink, Listera. The disruption in the DTR presumably lowered the extent of careful scrutiny of the sales pitch and induced a state of mindlessness, in which brand familiarity was used as a basis for (heuristic) decision making.

Though not significant, Figure 3.2 shows a slight trend of non-depleted participants tending to donate more money to the no authority organisation than to the authority organisation. Possibly the no authority organisation invoked more sympathy with participants because the organisation was described as a newcomer, which is generally more in need of support. If so, then perhaps the absence of a clear authority may foster the employment of alternative bases for judgment, such as the likeability principle. Although the difference is not significant, future research could further explore this possibility. 


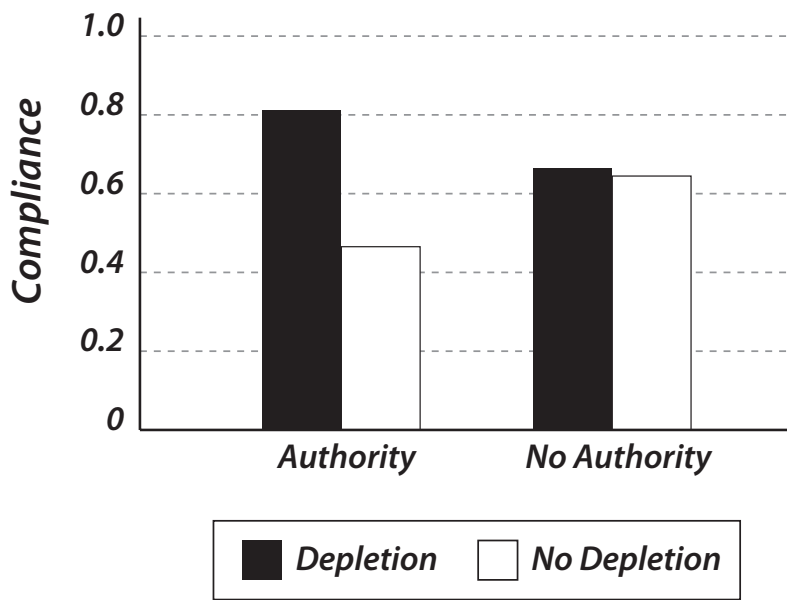

Figure 3.2. Percentage of money donated to charity, as a function of depletion-induction and heuristic-activation (adapted from Janssen et al., 2008).

In sum, these results provide converging support for the second stage of our model, the notion that regulatory resource depletion increases the odds of compliance with a target request, through the use of heuristics. That is, people comply with a request to a larger extent when their self-regulatory resources are low, provided that a heuristic is present in the influence setting.

\section{Experiment 3.3}

The purposes of Experiment 3.3 were to replicate the results of the previous experiments in a naturalistic setting, and once more include different manipulations of self-regulatory resource depletion and heuristic activation. We induced self-regulatory resource depletion with a mirror-tracing persistence task (see Quinn, Brandon \& Copeland, 1996) and activated the heuristic principle of likeability (Cialdini, 1993). The latter was done by giving participants a compliment on their task performance. We also assessed a different form of compliance: agreement to participate in future studies of a research society. We predicted that self-regulatory resource depleted participants would show increased compliance with this request, compared to non-depleted participants, but only when the likeability principle was made salient. 


\section{Design and procedure}

One hundred students ( 37 female, 63 male; mean age 21.54 years, $S D=2.39$ ) participated in a 2 (self-regulatory resource depletion condition: depletion vs. no depletion) X 2 (heuristic-activation: likeability vs. no likeability) between-subjects factorial design. One of five confederates (three female, two male) approached students on a university campus and asked whether they were willing to participate in a short study conducted by the (fictitious) Fluid Intelligence Society to test people's 'mental age.' Participants were randomly assigned to the depletion or no depletion condition.

Participants performed a geometric figure-tracing task (see Quinn et al., 1996), which required participants to hand-trace geometric figures for 4 minutes. In the depletion condition, participants performed this task with their nondominant hand and guided the movements of their hand by watching it in a mirror. Those in the no depletion condition traced the same figures with their dominant hand without the mirror.

After the tracing task, participants in the likeability condition were paid a compliment by the confederate ("you did a very good job performing this task"), a response that is known to activate the heuristic principle of likeability (Cialdini, 1993). In the no likeability condition, no comments about participants' task performance were made.

\section{Dependent measure}

Next, the confederate asked if participants would like to volunteer to participate in future studies of the Fluid Intelligence Society. Whether participants agreed to volunteer was our measure of compliance. Afterwards, participants were debriefed and thanked. 


\section{Results and discussion}

A fairly high percentage of participants (78\%) complied with the volunteering request, which was likely a result of the volunteering being described as fun to do. Given the dichotomous nature of the dependent variable, the data were analyzed using logistical regression. As predicted, and in line with the results of Experiments 3.1 and 3.2, there was a significant interaction between depletion induction and heuristic activation on compliance (Wald $(1)=5.36, p<.05)$. No other effects were observed.

Chi-square tests confirmed that self-regulatory resource depletion encouraged heuristic decision making: when the heuristic principle of likeability was activated, $88.5 \%$ of the participants who were depleted of their self-regulatory resources complied with the request as compared to $65.4 \%$ of participants in the no depletion condition $\left(\mathrm{X}^{2}(1)=\right.$ $3.90, p<.05)$. When the heuristic was absent from the influence setting, the percentage of depleted participants that complied was statistically equivalent to the percentage of non-depleted participants (70.8\% vs. $87.5 \%, x^{2}(1)=2.02, n s$, see Figure 3.3$)$.

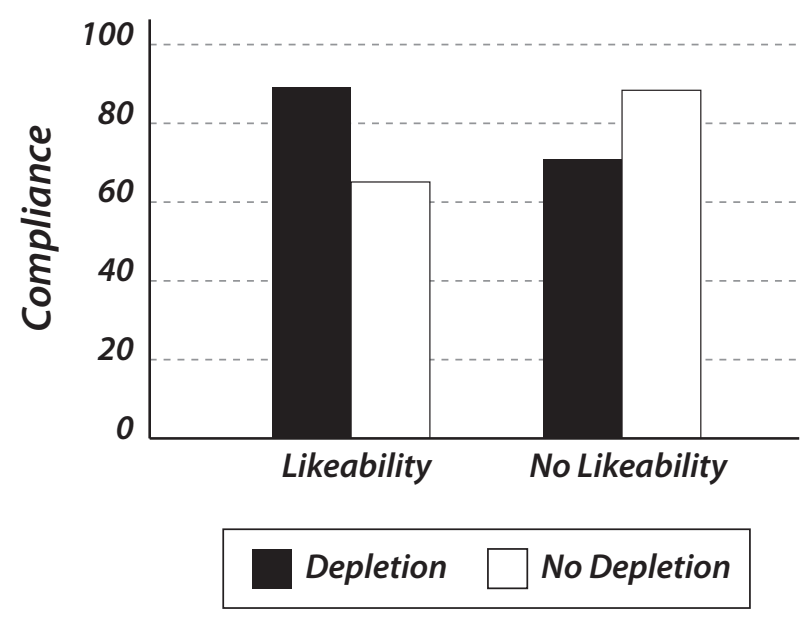

Figure 3.3. Percentage of participants willing to volunteer, as a function of depletion-induction and heuristic-activation (adapted from Janssen, Fennis, \& Pruyn, 2008). 
These results suggest that a state of self-regulatory resource depletion renders people susceptible to the likeability heuristic, thereby increasing the odds they will comply with a request. Importantly, the chi-square tests indicate that the interaction effect between self-regulatory resource depletion and likeability is ordinal, rather than disordinal, and thus parallels the findings of the previous experiments. Nevertheless, the pattern of results in the no-heuristic condition appears to suggest that depleted participants tended to comply less than non-depleted participants. Since the effect is nonsignificant we can only speculate as to the underlying processes, but one possibility is that the absence of a heuristic robbed depleted participants (but not their non-depleted counterparts) of a basis for making a decision, thus resulting in reduced compliance compared to non-depleted participants. That is, decision making requires self-regulatory resources (Vohs et al., 2008) and when people are temporarily weak in self-regulation they consequently become worse at making decisions (Pocheptsova, Amir, Dhar, \& Baumeister, 2009). Hence, it is possible that depleted participants who were left without a cue for making a decision did not have a salient guide to behavior. Conversely, for no depletion participants, their behavior may have served as a cue to subsequent compliance. That is, no depletion participants were given a fairly straightforward task (i.e., tracing figures without a mirror using their dominant hand). In the no likeability condition, no feedback on their performance was given and therefore these participants had to infer for themselves whether they had done a good job (again, the task was so easy that it was clear that they had). Given the easiness of the task, their performance may have prompted increased willingness to comply with the target request to volunteer in future studies, compared to no depletion participants in the likeability condition (who may have perceived the heuristic as redundant).

In sum, these findings extend the results of Experiments 3.1 and 3.2 to other types of heuristic principles, other manipulations of self-regulatory resource depletion, and other forms of compliance. Moreover, these results demonstrated that the impact of selfregulatory resource depletion on compliance is not restricted to the laboratory but can be elicited and observed in naturalistic dyadic influence settings as well. 


\section{Experiment 3.4}

Experiment 3.4, which was conducted in the laboratory, extends our previous results in an important way: whereas Experiments 3.1 to 3.3 relied on direct manipulations of selfcontrol depletion, the present investigation sought converging evidence by assessing individual differences in dispositional self-control (Tangney et al., 2004). To the extent that the proposed model is general, we ought to see not only that low self-regulatory resources can be induced by situational demands but also that people dispositionally low in self-control respond in a similar fashion. The heuristic principle that we manipulated is the principle of reciprocity that was also featured in Experiment 3.1, using a different manipulation in the current study. We predicted that participants low in dispositional self-control would be particularly susceptible to this heuristic, resulting in increased compliance with a request, which in this experiment was the willingness to do unpaid work for their university.

\section{Design and procedure}

A total of 104 undergraduate students ( 79 female, 25 male, mean age 20 years, $S D=1.73$ ) participated in return for partial course credit in a study with one between-subjects factor (heuristic-activation: reciprocity vs. no reciprocity) and trait self-control as the second independent variable. The current study was part of a larger set of studies that was introduced as a series of unrelated tasks.

Participants first completed the trait Self-Control Scale developed by Tangney et al. (2004). This instrument consists of 36 statements that are rated on a 5-point scale (1 $=$ not at all; $5=$ very much). Sample items include, 'I am good at resisting temptation', 'People would say that I have iron self-discipline,' and 'I often act without thinking through all the consequences' (reverse scored, see Tangney et al. [2004] for a complete listing of the items). The reliability of the instrument was satisfactory $(a=.85)$ and an 
index was created by averaging the scores on the items. Higher scores indicate higher levels of dispositional self-control.

Since the current study was part of a larger set of studies, a short study was conducted in between the self-control questionnaire and the rest of the experiment, functioning as a filler task, as to obscure the real purpose of the study. Following this filler study, the experiment continued with a procedure to activate the heuristic principle of reciprocity. In the reciprocity condition, participants were done a favor by their university: participants were informed that their university had planned to temporarily lower the prices of different food products in the cafeterias on campus, due to a different caterer taking over this service. They were also informed that their university decided upon providing higher rewards for students participating in scientific research conducted by the university. Participants were asked to indicate to what extent they approved of these measures (that were supposedly discussed in the latest meeting of the board of governors of their university) on a 9-point scale ( $1=$ not at all; $9=$ very much). Mean scores on these scales were $6.94(S D=2.17)$ and 6.30 (SD = 2.35), respectively, indicating that overall there was rather high agreement on these matters, as expected. Participants in the no reciprocity condition were not provided with this favor by their university; they were not informed about the lowering of food prices and higher rewards for research participation and were not asked to give their opinion about these measures. The concession made by the participants' university, lowering food prices and increasing rewards for participating in scientific research, was aimed at inducing a counter concession on the part of the participant (Cialdini, 1993) in the form of increased compliance with an upcoming request of their university.

\section{Dependent measure}

Next, participants read a message on their computer screen, informing them that the board of governors of their university had also decided upon expanding the university's 
(existing) student employment agency. In addition to offering paid jobs (e.g., working as a research assistant), the agency was now recruiting students for unpaid jobs as well (e.g., providing support as a car-park attendant, traffic controller, or cloakroom assistant during campus events). Participants were asked to indicate on a scale ranging from 0 to 24 hours in 2-hour intervals how much time per year they would be willing to do unpaid work for their university. Length of time participants were prepared to volunteer was our measure of compliance. Afterwards, participants were debriefed and thanked for their participation.

\section{Results and discussion}

Overall, $66 \%$ of participants agreed to act as a volunteer in response to the target request. A multiple regression with number of hours prepared to volunteer as the criterion and heuristic-activation (reciprocity vs. no reciprocity, dummy coded), trait self-control (a continuous, centered predictor), and their interaction as predictors, revealed a significant interaction between heuristic activation and trait self-control $(\beta=-.26, t(100)=-2.10, p<.05)$. In line with our hypothesis, a simple slopes analysis (Aiken \&West, 1991) revealed that the impact of the reciprocity heuristic on compliance was significant among participants low in dispositional self-control (evaluated at one standard deviation below the mean, $\beta=.35, t(100)=2.62 p<.05)$ and was not significant among participants high in dispositional self-control (evaluated at one standard deviation above the mean, $\beta=-.05, t<1$, see Figure 3.4). The main effects of heuristicactivation and trait self-control were not significant $(\beta=.15, t=1.60, n s ; \beta=-.12, t<1$, respectively). 


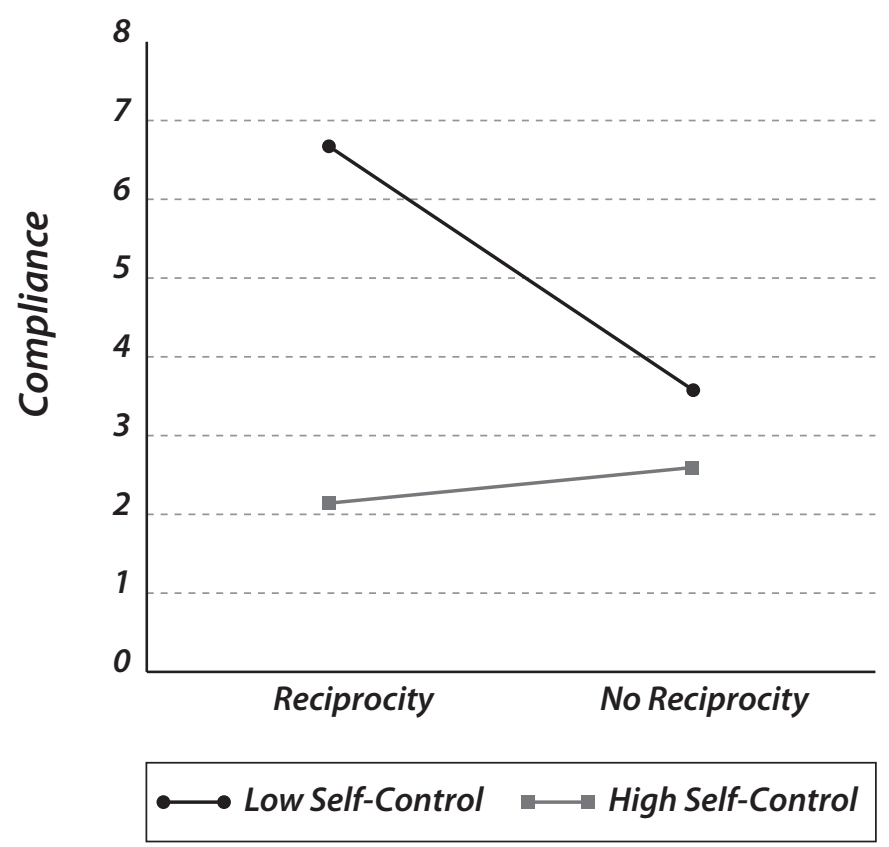

Figure 3.4. Average number of hours prepared to volunteer as a function of heuristic-activation and trait self-control.

These results corroborate the second hypothesis of our two-stage model, which states that lower levels of self-control foster compliance but only to the extent that the influence context harbors a powerful heuristic. People with a lower tendency for selfcontrol proved to be especially open to the reciprocity principle.

\section{General discussion}

The present research tested the second premise of our two-stage model to account for the role of consumer self-regulatory resource depletion in the effectiveness of sequential request scripts that are used by compliance professionals to elicit compliance. In four studies we found support for Stage 2 of the model, which posits that a reduced supply of self-regulatory resources fosters compliance with a target request, but not by default. Rather, it is posed to do so through an overreliance on salient heuristics that facilitate compliance as an efficient behavioral response. We consistently found 
an effect of reduced self-control fostering reliance on several different heuristics that are frequently embedded in social influence techniques (reciprocity in Experiments 3.1 and 3.4, authority in Experiment 3.2, and likeability in Experiment 3.3), increasing the odds of compliance with several different requests (volunteering as an experimenter during scientific research, volunteering for a research society, doing unpaid jobs for the university, and donating actual money to a charity organization). These experiments were conducted in the lab as well as in a field setting, and all used different ways to induce a state of self-regulatory resource depletion, with Experiment 3.4 focusing on individual differences in self-control.

Converging evidence that low dispositional self-control increases susceptibility to influence was found in a study by Fennis et al. (2009, Experiment 6). This experiment was conducted in the lab, presenting participants with a full sequential request technique exposed of an initial and a target request, or presenting them with a target request only. The influence technique that was administered was the low-ball technique (Burger \& Petty, 1981; Cialdini et al., 1978). The heuristic principle of consistency (Burger \& Cornelius, 2003; Cialdini, 1993; Cialdini et al., 1978) drives the effectiveness of this procedure. In the low-ball technique, people are presented with an initial request (e.g., "Would you like to participate in a study?") and after they comply, the cost of compliance is raised (e.g., "The study will be conducted next Sunday at 7.00 a.m."). The act of initial compliance activates the principle of consistency, which in turn fosters compliance with the intended target request. Germane to our notions regarding the first stage of our two-stage model, research by Burger and Petty (1981) suggests that the tendency to behave consistently is primarily the result of having to speak aloud to the influence agent one's agreement with the initial request, which likely prompts impression management concerns (e.g., participants may feel they cannot 'let down' the requester when the target question is raised). According to Burger and Petty (1981), this sense of commitment is primarily felt toward the requester rather than the request itself, and thus the act of initial compliance in the low-ball procedure may evoke effortful self- 
presentation vis-à-vis the influence agent. In this sense, the low-ball technique shares crucial characteristics with many other social influence techniques albeit stemming from a different source (i.e., through an unfulfilled obligation to the requester rather than commitment to the initial responses). The hypothesis underlying the study by Fennis et al. (2009, Experiment 6) was that the low-ball technique would be particularly effective among people low in self-control. This hypothesis was supported by the study, where compliance was measured as money donated to a charitable cause. In particular, participants exposed to the low-ball technique donated more money than participants in a target-request-only condition, but simple slopes analysis specified this effect only to be significant among participants low in dispositional self-control. In contrast to Experiment 3.2 as presented in the current chapter, where participants were given money in the beginning of the study as payment for their participation, the participants in this study reached into their pocketbooks and donated, as they so desired, their own money to the charity presented to them.

Taken together, there is ample evidence for the second premise of our model. Five studies showed that weak self-regulation can lead to acquiescent behavior in the presence of salient norms or heuristic principles that inform the weakened participant to do so. In addition, the convergence of findings between the laboratory and the field demonstrated that it is not merely a self-selection phenomenon that explains who becomes compliant (i.e., thoughts that only 'those types' of people would fall for influence tactics). The field study (Experiment 3.3) adds to the generalizability of the laboratory findings by showing that a community sample of consumers showed patterns of compliance comparable to those of our undergraduate student sample.

The present work extends the literature on social influence by focusing on the very act of compliance, a dependent variable that all too often has been taken for granted in earlier studies. A recent analysis of research in social and personality psychology concluded that there is a dearth of behavioral research in the past two decades, most 
likely because behavioral studies are costly and difficult to pursue particularly when one needs multiple studies to make one's point convincingly (Baumeister, Vohs, \& Funder, 2007). The compliance effects in our work pertain to real, overt behavior, instead of selfreports.

Apart from shedding light on the dynamics underlying diverse acts of compliance as brought about by a sequential request technique, our work also extends previous research on the behavioral effects of regulatory resource depletion. Earlier studies have stressed that self-regulation failure often results in egocentric, self-serving, and sometimes even antisocial behavior (Finkel \& Campbell, 2001; Tangney et al., 2004). Our model allows for an alternative behavioral outcome, namely that self-regulation failure may also produce prosocial behavior if a suitable context (i.e., heuristic) that fosters compliance is present.

In sum, the present research offers an explanation for the mindlessness so often observed in social influence situations. When self-regulatory resources have been lowered during the initial stage of a sequential request social influence technique (as tested in Chapter 2 of this dissertation), people more easily resort to salient heuristics for decision making, thereby increasing the chance that they will comply with a request to invest time, effort, or money. The studies in Chapters 2 and 3 tested a two-stage model to account for the role of consumer self-regulatory resource depletion in the effectiveness of sequential request scripts that are used by influence professionals to elicit compliance. Our model holds that these social influence strategies comprise a series of requests that trigger one underlying process: self-regulatory resource depletion. The two-stage model posits that responding to an involving initial request (be that answering a series of cognitively demanding questions or questions that prompt self-presentational responses) reduces the supply of self-regulatory resources within the target of influence. A reduced supply of regulatory resources, in turn, fosters compliance with the target request, but not by default. Rather, it is posed to do so through an overreliance on salient heuristics 
that facilitate compliance as an efficient behavioral response. Hence, responding in an effortful way to an initial request induces self-regulatory resource depletion, which subsequently encourages heuristic decision making. In dyadic influence settings aimed at fostering compliance, the product of this decision-making process is donating money, time, or effort.

The findings of eight experiments reported in Chapters 2 and 3 of this dissertation, as well as converging evidence provided by four other studies (Experiments 1, 2 and 6 from Fennis et al., 2009 and a study by Fennis \& Janssen, under review), together employing a total of 836 influence interactions, support the tenets outlined in our two-stage model.

In the next chapter we report research that extends the two-stage model and highlights the motivational aspects of self-control. Whereas Chapters 2 and 3 focused on the role of self-control ability in social influence situations and point to compliance as a consequence of this ability being low, Chapter 4 stresses resistance as a more likely outcome of a social influence process when self-control ability is high, or when one is motivated to become efficient in the use of remaining resources when self-control ability is not optimal. When resources for self-regulation are low, regulatory efficiency seems to be key. We predicted that individuals low in self-control resources can still be successful at defending themselves against a persuasive attack, when prompted to be efficient in allocating their remaining self-regulatory resources. 



\section{Chapter 4}

Extending the model:

Forewarned is forearmed:

Conserving self-control strength

to resist social influence ${ }^{3}$ 

o you have any idea how often complete strangers have persuaded you to do them a favor, without any prospect of them doing you a favor in return? And how often have you complied with their requests when you had no initial intention to do so? Probably more often than you would like to admit. Although there is nothing wrong with showing some compliance once in a while, for instance when a fundraiser asks you for a donation for a good cause, it is hardly functional to comply with every request that you receive. Resisting influence attempts from advertisers, telemarketers, salespeople, and fundraisers is growing into an everyday phenomenon and saying "No" is vital if we want to live our lives in peace. But resisting persuasion is not easy, since we are simply not always aware of the persuasive intent of a request or message, or we otherwise lack the ability or the motivation to resist a persuasive appeal (e.g., Briñol \& Petty, 2005).

As recent research has shown (and referring to the previous two empirical chapters of this dissertation), resisting persuasion is frequently a costly process that involves active selfregulation. Resisting an influence attempt consumes self-regulatory resources, with the inevitable consequence that when these resources are low, one's attempts at resistance are more likely to fail (Burkley, 2008; Fennis et al., 2009; Janssen, Fennis, Pruyn, \& Vohs, 2008; Wheeler et al., 2007). Hence, resisting (unwanted) influence is more successful when self-regulatory resources are high, rather than low, but the present research demonstrates that all is not lost for those in a state of self-regulatory resource depletion. Under certain conditions, depleted individuals can successfully conserve the resources they have left to be put into action when encountering an unwanted persuasive attack. In the present research we will argue that forewarning people of an impending influence attempt may serve to promote self-regulatory efficiency and thus prompt depleted individuals to conserve what is left of their regulatory resources. As a result, when these individuals are subsequently confronted with a request, they will comply less than their depleted counterparts who were not forewarned and did not conserve their resources, and they will be as able as non-depleted participants to resist the influence attempt. 
According to the limited-resource model of self-control (Baumeister et al., 1998; Muraven et al., 1998; Vohs \& Heatherton, 2000; for a review, see Baumeister, Vohs, \& Tice, 2007), any act of deliberate and regulated responding by the self, such as overriding impulses, active choice and controlled (as opposed to automatic) processing, draws on a limited intrapsychic resource. Akin to strength or energy, this resource becomes depleted with use, and is recovered slowly. Many studies have shown that self-control ability suffers after previous exertion of willpower or self-control (e.g., Baumeister et al., 1998; Muraven et al., 1998; Schmeichel et al., 2003). In a state of self-regulatory resource depletion, the self resorts to more passive and low-effort courses of action (e.g., Baumeister et al., 1998), thereby increasing vulnerability to untoward impulses, habit, and automatic processes (Baumeister et al., 2000; Baumeister \& Vohs, 2007; Vohs et al., 2005).

Recently, research started to test the notion that resisting persuasion is an activity which also draws on limited regulatory resources, and it showed that a state of selfregulatory resource depletion weakens resistance to temptations and (unwanted) influence attempts (Baumeister, 2002; Vohs \& Faber, 2007). In a study by Wheeler et al. (2007), participants previously depleted of their self-control resources by an unrelated task showed less resistance to a counterattitudinal persuasive message; they reported more acquiescent attitudes and generated fewer counterarguments than their non-depleted counterparts, especially when message arguments were weak. In line with dual-process frameworks (Chaiken \& Trope, 1999), depletion of selfcontrol resources appeared to inhibit the generation of counterarguments because it hindered processing of message-relevant information, as evidenced by reduced sensitivity to argument quality. Similar to Wheeler et al. (2007), Burkley (2008) showed that persuasion by a counterattitudinal message increased after an act of selfcontrol, and more resistance toward a persuasive message diminished the amount of self-control resources available to use on a subsequent unrelated self-control task. 
Another demonstration of the role of self-regulatory resources in resisting and yielding to social influence was presented by Fennis et al. (2009) and Janssen, Fennis, Pruyn, and Vohs (2008). They forwarded self-regulatory resource depletion as an important underlying factor mediating the effectiveness of social influence techniques, such as a foot-in-the-door (FITD), door-in-the-face (DITF), or low-ball technique (Burger \& Petty, 1981; Cialdini et al., 1975; Freedman \& Fraser, 1966). The authors argued that a specific feature of such techniques promotes self-regulatory resource depletion, which paves the way for consumer compliance. More specifically, all these techniques are made up of a sequence of requests, starting with an initial request or series of initial requests (that can either be small, as in the FITD, large, as in the DITF, or particularly attractive, as in the low-ball procedure), and culminating in a target request for which compliance is sought. They showed that actively responding to the initial request of this sequence depleted self-control resources. The resulting state of weak self-control ability increased compliance with a subsequent (charitable) target request, such as freely donating time, effort, or money. Importantly, depleted participants were not susceptible to influence by default, but rather because they relied more on compliance-promoting heuristics that were present in the persuasion context, such as authority, reciprocity, or likeability (cf. Cialdini's principles of influence, 1993).

In sum, and in line with dual-process models of persuasion (e.g., Petty \& Wegener, 1999), a state of self-regulatory resource depletion reduces systematic or central-route processing, and enhances the weight on heuristic processing in consumer judgment and decision making. This renders it more difficult to resist (unwanted) influence attempts, an activity which evidently requires systematic processing to engage in issuerelevant thinking and argue against the persuasive communication. A key means to encourage more systematic processing and thereby increase the odds of resistance to persuasion could be to increase people's awareness of an upcoming influence attempt in advance. Multiple studies have suggested that forewarning people of an upcoming persuasive communication motivates them to counterargue the message in order to 
reassert their attitudinal freedom, and thereby increase resistance to persuasion (e.g., Allyn \& Festinger, 1961; Brock, 1967; Chen, Reardon, Rea, \& Moore, 1992; Freedman \& Sears, 1965; Hass \& Grady, 1975; Kiesler \& Kiesler, 1964; Petty \& Cacioppo, 1977; see Wood \& Quinn, 2003 for a review). For instance, in an experiment by Petty \& Cacioppo (1977), forewarning undergraduate students of the content of an upcoming discrepant communication (a tape recording by their faculty committee, recommending that university seniors should be required to pass a comprehensive exam in their major prior to graduation) stimulated anticipatory counterargumentation, and resulted in less persuasion.

We may assume that a forewarning of an influence attempt can be an effective means to increase resistance, because it encourages biased, systematic scrutiny of the persuasive message as evidenced by increased counterargumentation. But what if the motivation to counterargue is present, but the ability to engage in counterargumentation is lacking because of previous depletion of regulatory resources? We argue that in these conditions, forewarning motivates people to conserve their remaining resources, and mobilize them in the service of this counterargumentation. This presupposes that a depleted state does not reflect a complete exhaustion of resources but rather a temporary or relative deficit, a notion that was recently supported by Muraven et al. (2006). They suggested that individuals are motivated (at either a conscious or an unconscious level) and able to conserve their regulatory energy when the benefits of using the resource in the future apparently outweigh the benefits of using it right now. Their studies showed that expecting to exert self-control in the future motivated participants who exerted self-control in the past (and were thus depleted of their regulatory resources) to conserve their remaining self-control strength for this future task. These participants performed worse on an intervening measure of self-control than participants who were either not depleted, or not expecting future self-control. Moreover, when performance on this future task was actually measured, participants who were initially depleted but conserved resources performed as well as non-depleted participants. In contrast, initially depleted participants not expecting to exert self-control in the future performed worse than participants in the other conditions 


\section{The present research}

In sum, previous research has shown that a state of self-regulatory resource depletion weakens resistance to (unwanted) influence attempts, and studies have shown that individuals low in self-control strength are able to conserve what is left of their resources for future use. Extending these two lines of research, the present research argues that forewarning people of persuasion may foster self-regulatory efficiency by motivating them to conserve self-control strength to resist the upcoming influence attempt. We expect that a forewarning of an influence attempt prompts individuals to conserve what is left of their resources, but mainly when there is a clear rationale for doing so, i.e., when they previously suffered a loss of self-control resources. Since self-regulatory resource depletion has been found to have an acquiescing effect on compliance with a request (Fennis et al., 2009; Janssen, Fennis, Pruyn, \& Vohs, 2008), a motivation to conserve resources would be especially beneficial to those low in self-control strength. As compared with others who are less depleted, they should try to avoid expending more strength so that they can build up their resistance. By contrast, we do not expect forewarning to promote self-regulatory efficiency when people's resources have not previously been depleted by an act of self-control; since one would have enough resources left for resisting a future influence attempt, there should be no direct need for conservation. As a result of this (seemingly unconscious) process of conserving strength, we expect depleted participants who were forewarned of an influence attempt to comply less with a subsequent persuasive request than their depleted counterparts who were not forewarned and thus did not conserve their resources. Moreover, we expect them to be as able as non-depleted participants to resist the influence attempt.

As such, the present research contributes to the literature in four key ways. First, it shows that self-regulatory efficiency may play an important role in resisting social influence. Second, it provides insight into the self-regulatory dynamics underlying 'classic' effects of forewarning on persuasion. Third, by linking up self-control resources with dual- 
process models of persuasion, the present research underscores the self-regulatory mechanisms driving systematic or central information processing in response to persuasion attempts. Fourth, it extends previous work on resistance and persuasion by showing that the effects of forewarning of persuasive intent are not only a function of the recipients' motivation, but also of their ability to withstand an unwanted upcoming influence attempt.

We conducted three experiments to test our hypotheses. In a first study we tested the effect of depletion and forewarning of an influence attempt on compliance with a persuasive request. We expected that a forewarning would decrease the amount of compliance with the request, but mainly among previously depleted individuals. In a second study we aimed to demonstrate that the process underlying this effect is one of conservation of remaining self-control resources. We assumed that people forewarned of an influence attempt would be motivated to conserve self-control strength for this future persuasive encounter, reflected in reduced performance on an intermediate selfcontrol task, but mainly when some of their resource had previously been depleted. In line with Muraven et al. (2006), we expected that conserving resources would eventually be beneficial. When subsequently confronted with a request, depleted participants who saved their resources should be just as able as non-depleted participants to resist this influence attempt by showing less compliance. In a third study we more directly assessed the process of resistance to persuasion. We tested whether a forewarning would also prompt depleted individuals to conserve their resources in the service of counterarguing a persuasive request. 


\section{Experiment 4.1}

\section{Design and procedure}

One hundred and thirteen undergraduate students (92 female, 21 male; mean age 21.27 years, $S D=2.82$ ) participated in exchange for partial course credit. The study used a 2 (self-regulatory resource depletion condition: depletion vs. no depletion) X 2 (forewarning: forewarning of an influence attempt vs. no forewarning) betweensubjects factorial design.

On arrival at the laboratory, participants were seated in individual cubicles fitted with a desktop computer, which presented all the instructions. Participants were randomly assigned to one of the four conditions, and informed that the experiment consisted of several different, unrelated tasks.

We induced a state of self-regulatory resource depletion with a self-control task adopted from Muraven et al. (2006). All participants were shown a paragraph of typed, dense text (contents were copied from a highly advanced statistics book), and were instructed to retype the paragraph as quickly as possible in a textbox below. In contrast to participants in the no depletion condition, who were simply instructed to retype the entire text, participants in the depletion condition had to retype the paragraph without using the letter $e$ and the space bar. Applying such a rule has been shown to require self-control, because one has to actively override the natural inclination to type every letter (Muraven et al., 2006).

Next, serving as a manipulation check for the depletion-inducing typing task, we assessed participants' self-control efforts: on 9-point scales ( $1=$ not at all; $9=$ very much), participants indicated to what extent they found the task difficult and effortful, how much they needed to control themselves during the task, and how much energy 
they spent on suppressing automatic responses during the task. To be able to rule out a possible effect of the typing task on participants' emotions, we also asked them to indicate how much they enjoyed the task.

Half of the participants read a message on their computer screen, forewarning them about an upcoming influence attempt. The message stated: 'We would like to call to your attention that after you have finished this study, a representative of the student project 'Campus Clean' will drop by to present you with some information about their activities. They are actively seeking volunteers for the new academic year, and would like to persuade you to donate a few hours of your time to participate in their project.' The other half of the participants did not receive any forewarning. All participants subsequently performed a filler-task, to ascertain that the forewarning would not be directly followed by our compliance measure.

\section{Dependent measure}

All conditions were presented with the charitable cause for which participants' compliance was sought. Participants were informed about the goals and activities of the Campus Clean student volunteering project. Note that participants in the forewarning condition had already been made aware of the existence of this project, but for participants in the no forewarning condition the project was introduced at this stage. All participants were presented with the following information: 'We would like to inform you about the activities of the Campus Clean project, which started this academic year. A group of students of this university has taken the initiative to get together after lectures and examinations to collect trash, like food-wrappers, soda cans and bottles, left behind in the main lecture halls of the campus buildings. This way, all lecture areas will stay fresh and tidy during lecture hours, which will also be in your own personal interest.' Note that the last sentence about the Campus Clean project was framed as a claim for reciprocity: according to our two-stage model, that was tested in the previous 
two chapters of this dissertation, self-regulatory resource depletion fosters compliance, but not by default: it is posed to do so through an overreliance on salient heuristics that facilitate compliance as an efficient behavioral response. Therefore, the information about the Campus Clean project was accompanied by a claim for reciprocity, in the previous chapter proven to be a strong decisional heuristic especially for participants low in regulatory resources.

The project information was followed by the actual request. Participants were asked whether they would be willing to act as a volunteer for Campus Clean. Specifically, they were asked to indicate how much time they would be willing to spend clearing up their lecture halls during a full academic year. They could respond to this request on a scale ranging from 0 to 240 minutes in 15-minute intervals. The amount of time participants indicated to volunteer served as our measure of compliance (cf. Fennis et al., 2009; Kardes et al., 2007). Afterwards, participants were fully debriefed and thanked.

\section{Results and discussion}

Manipulation check. T-tests showed that participants in the depletion condition who were asked to retype the paragraph without using the letter $e$ and the space bar, considered this task more difficult $(M=5.35, S D=2.19, t(111)=4.16, p<.001)$ and effortful $(M=5.85$, $S D=1.93, t(111)=2.41, p<.05)$, than participants in the no depletion condition who simply retyped the entire text $(M=3.66, S D=2.13 ; M=4.95, S D=2.06$, respectively). In addition, participants in the depletion condition indicated that they had to put more effort into controlling themselves during the task $(M=5.80, S D=2.11, t(111)=2.64, p<.05)$ and suppressing automatic responses $(M=5.98, S D=2.44, t(111)=5.67, p<.001)$ than participants in the no depletion control condition $(M=4.76, S D=2.09 ; M=3.59, S D=2.05$, respectively). Hence, our manipulation of self-regulatory resource depletion proved successful. Moreover, the task probably did not induce unwanted mood effects, as applying a more complex rule did not affect enjoyment of the task compared to a simpler task $(t<1)$. Thus, it is unlikely 
that participants in the self-regulatory resource depletion condition would respond differently to the subsequent request for compliance because it affected their emotions.

Compliance. Overall, $62.8 \%$ of participants agreed to act as a volunteer in response to the request. An ANOVA was conducted on participants' amount of compliance with the request, as a function of self-regulatory resource depletion condition (depletion vs. no depletion) and forewarning (forewarning of an influence attempt vs. no forewarning).

The ANOVA showed a main effect of both factors. In accordance with the findings of Fennis et al. (2009), participants who were depleted of their regulatory resources were willing to volunteer for a larger amount of time $(M=58.91, S D=66.45)$ than participants in the no depletion condition $(M=39.83, S D=52.16), F(1,109)=5.22, p$ $<.05, d=.32$. Furthermore, participants forewarned about the upcoming influence attempt of a Campus Clean representative showed more resistance: they complied far less with the request to volunteer for this project $(M=28.28, S D=38.15)$ than participants not forewarned $(M=73.56, S D=71.25), F(1,109)=21.39, p<.001, d=.79$.

Of main importance for our hypothesis, the analysis also showed the predicted interaction between self-regulatory resource depletion and forewarning, $F(2,109)=5.87$, $p<.05, \eta^{2}=.04$. Additional simple main effects analyses showed that forewarning of an influence attempt increased resistance to compliance among depleted individuals. As shown in Table 4.1, when depleted, forewarned participants complied far less with the request to voluntarily clear up their lecture halls as compared to their non-forewarned counterparts, $F(1,109)=24.02, p<.001, d=1.22$. Among non-depleted participants, however, the effect of forewarning did not reach significance, $F(1,109)=2.51$, ns. These results extend previous research in showing that although a temporary lapse in selfregulatory resources makes one more susceptible to compliance with a request (Fennis et al., 2009; Janssen, Fennis, Pruyn, \& Vohs, 2008), forewarning of the influence attempt may function to conserve self-regulatory resources and result in increased resistance. 
Table 4.1

Average number of minutes participants volunteered as a function of resource depletion and forewarning

\begin{tabular}{cccccccc}
\hline \multicolumn{4}{c}{ Depletion } & \multicolumn{4}{c}{ No Depletion } \\
\hline \multicolumn{2}{c}{ Forewarning } & \multicolumn{2}{c}{ No Forewarning } & \multicolumn{2}{c}{ Forewarning } & \multicolumn{2}{c}{ No Forewarning } \\
\hline$M$ & $S D$ & $M$ & $S D$ & $M$ & SD & $M$ & SD \\
\hline $27.58 a$ & 37.57 & $99.38 b$ & 74.12 & $29.00 a$ & 39.38 & $51.43 a$ & 61.70 \\
\hline
\end{tabular}

Note. Means that do not share subscripts differ at $\mathrm{p}<.05$.

In sum, Experiment 4.1 showed that forewarning of an influence attempt increases resistance for initially depleted individuals, up to the level of non-depleted individuals. A second study was performed to uncover the assumed underlying psychological process by showing that it is indeed a matter of conserving self-control strength that drives this effect. Therefore, Experiment 4.2 included a validated measure of selfregulatory resource depletion, to test the notion that forewarning directly affects self-regulatory resources, which can be used to ward off unwanted influence. We expected initially depleted participants who were forewarned to show more reduced performance than all other participants on this intermediate self-control measure; since they have already suffered a loss of self-control, they should be most concerned with conserving their remaining self-control resources for the upcoming influence attempt. Second, we wanted to rule out the alternative explanation that receiving any preliminary information about an upcoming encounter, rather than an explicit forewarning, accounts for the effect. Therefore we added a second control condition to our forewarning manipulation, in which participants were given the same preliminary information about the Campus Clean student project as participants in the forewarning condition, but without forewarning them of the upcoming request to volunteer for the project. We expected the results of this condition to parallel the findings of participants who were not given any preliminary information or forewarning. In sum, for those low in self-control, a forewarning should motivate them to conserve resources only when 
anticipating being exposed to influence, since self-control resources are required to resist the upcoming persuasive appeal. Providing people with information about the topic of influence should not be enough to generate the effects. As in Experiment 4.1, and as a direct result of conservation of resources, forewarning people of an impending influence attempt should increase resistance to compliance among depleted individuals.

\section{Experiment 4.2}

\section{Design and procedure}

One hundred and seventeen undergraduate students (86 female, 31 male; mean age 19.77 years, $S D=1.72$ ) participated in exchange for partial course credit. The study used a 2 (self-regulatory resource depletion condition: depletion vs. no depletion) X 3 (forewarning: forewarning of an influence attempt vs. no forewarning-preliminary information vs. no forewarning-control) between-subjects factorial design.

On arrival at the laboratory, participants were seated in individual cubicles fitted with a desktop computer, which presented all the instructions. Participants were randomly assigned to one of the six conditions, and informed that the experiment consisted of several different, unrelated tasks. For most part, the procedure parallels that of Experiment 4.1.

We induced a state of self-regulatory resource depletion using the self-control task from Experiment 4.1. Participants retyped a paragraph as quickly as possible, yet those in the self-regulatory resource depletion condition were instructed to retype the paragraph without using the letter $e$ or the space bar. 
Next, participants read a message on their computer screen, informing them about the upcoming parts of the study. All participants read that the next task would be to solve a series of mathematical progressions. In the no forewarning-control condition the message ended here. Paralleling Experiment 4.1, participants in the forewarning condition were additionally informed about an upcoming encounter with a representative of the Campus Clean student project, as were participants in the no forewarning-preliminary information condition: 'We would like to call to your attention that after you are done with this study, a representative of the student project Campus Clean will drop by to present you with some information about their activities.' Only for participants in the forewarning condition, this message contained a forewarning, which was the same as in Experiment 4.1: 'They are actively seeking volunteers for the new academic year, and would like to persuade you to donate a few hours of your time to participate in their project.'

\section{Dependent measures}

Self-regulatory resource depletion. After reading one of the three messages explained above, participants were asked to solve three moderately difficult mathematical progressions to measure whether they would conserve their self-control resources when forewarned about an upcoming influence attempt. A sample item includes: 'Which number logically comes next in this string of numbers: $1-2-3-5-8-\ldots$ ' (The correct answer is 13; each succeeding number is the sum of the previous two numbers). Previous research has shown that performance on this type of advanced cognitive processing is susceptible to impairment due to prior resource depletion (Schmeichel et al., 2003). The number of correctly solved progressions served as our measure of self-control performance.

Compliance. Similar to Experiment 4.1, the amount of time participants were prepared to voluntarily clear up their lecture halls for the Campus Clean student project (measured in 15 min. intervals) served as our measure of compliance. Afterwards, participants were debriefed and thanked. 


\section{Results and discussion}

Self-regulatoryresourcedepletion. An ANOVA was conducted on participants'performance on the mathematical progressions, as a function of self-regulatory resource depletion condition (depletion vs. no depletion) and forewarning (forewarning of an influence attempt vs. no forewarning-preliminary information vs. no forewarning-control). This analysis showed a main effect of both factors. As expected, and in accordance with previous depletion research (e.g., Baumeister et al., 1998; Muraven et al., 2006), participants who performed the rule version of the e-task provided fewer correct answers to the mathematical progressions $(M=1.83, S D=.82)$, and were thus more depleted as compared to participants in the no depletion condition, who did not have to apply complex rules $(M=2.14, S D=.75), F(1,111)=5.05, p<.05, d=.39$. There was also a significant effect on math-performance for the three different forewarning conditions, $F(2,111)=4.72, p<.05, \eta^{2}=.07$. Post-hoc LSD comparisons indicated that participants who were forewarned of an influence attempt performed worse on the mathematical progressions $(M=1.70, S D=.79)$ as compared to participants who only received preliminary information about the Campus Clean project $(M=2.05, S D=.78, p<.05$, $d=.45)$ and participants who did not receive a forewarning or preliminary information $(M=2.20, S D=.76, p<.01, d=.65)$. The no forewarning-preliminary information and no forewarning-control conditions did not differ in logical math performance, $p=.39, n s$.

Most important, the analysis also showed the predicted interaction effect between selfregulatory resource depletion and forewarning, $F(2,111)=3.83, p<.05, \eta^{2}=.06$. Please note that we expected depleted participants who were forewarned of an upcoming influence attempt to maximally conserve their remaining self-control resources, compared to the other conditions, who were either not forewarned or not initially depleted. Therefore, we expected depleted and forewarned participants to show the poorest performance on the mathematical progressions, since they would be most concerned with conserving their remaining resources to resist the upcoming influence attempt. Hence, we predicted 
a significant contrast between the depletion-forewarning condition and the remaining (five) conditions that were not expected to differ significantly from each other. To test this hypothesis, we used a planned contrasts procedure outlined by Bobko (1986) for testing ordinal interactions. This procedure states that two interaction contrasts should be performed. The first contrast tests the equality of the conditions whose means are assumed to be equivalent, using a one-way analysis of variance. The second contrast tests whether the average of these conditions significantly differs from the one condition that is assumed to perform differently, using a planned comparison t-test (see Bobko, 1986 for a more extended discussion). For the first contrast, we included the depletion-no forewarning-preliminary information, depletion-no forewarning-control, no depletionforewarning, no depletion-no forewarning-preliminary information, and no depletionno forewarning-control conditions in the one-way analysis of variance. As expected, this analysis showed the five means to be statistically equivalent, $F(4,96)=1.13$, ns. Hence, these five groups performed similarly on the self-control math task, indicating that there was no differential tendency to conserve self-regulatory resources. The second contrast tested whether depleted participants anticipating an upcoming influence attempt performed worse on the logical math test than the average of participants in the other five conditions, using a planned comparison t-test. This analysis confirmed our prediction that depleted and forewarned participants conserved their self-control strength to a higher extent than participants in any of the other conditions, $t(111)=$ 4.15, $p$ <.001. As shown in Table 4.2, inspection of the means corroborated this result: a series of simple contrast analyses, with the 'depletion-forewarning condition' as the referent category, confirmed that participants in this condition indeed performed worse on the mathematical progressions than participants in the depletion-no forewarningpreliminary information condition $(t(111)=-2.00, p<.05, d=.66)$, the depletion-no forewarning-control condition $(t(111)=-4.03, p<.001, d=1.36)$, the no depletionforewarning condition $(t(111)=-2.97, p<.01, d=.98)$, the no depletion-no forewarningpreliminary information condition $(t(111)=-3.82, p<.001, d=1.30)$, and the no depletionno forewarning-control condition $(t(111)=-3.18, p<.01, d=1.02)$. Hence, for depleted 
participants, it was the forewarning, rather than receiving preliminary information per se that affected their management of remaining self-regulatory resources.

Table 4.2

Average number of correctly solved mathematical progressions as a function of resource depletion and forewarning

\begin{tabular}{lcccccc}
\hline & \multicolumn{2}{c}{ Forewarning } & \multicolumn{2}{c}{ Preliminary Information } & \multicolumn{2}{c}{ No Forewarning } \\
\hline & $M$ & $S D$ & $M$ & $S D$ & $M$ & $S D$ \\
\hline Depletion & $1.35 b$ & .67 & $1.83 a$ & .79 & $2.30 a$ & .73 \\
\hline No Depletion & $2.05 a$ & .76 & $2.26 a$ & .73 & $2.10 a$ & .79 \\
\hline
\end{tabular}

Note. Means that do not share subscripts differ at $\mathrm{p}<.05$

Compliance. Overall, $60.7 \%$ of participants agreed to act as a volunteer in response to the request. An ANOVA was conducted on participants' amount of compliance, as a function of self-regulatory resource depletion condition (depletion vs. no depletion) and forewarning (forewarning of an influence attempt vs. no forewarning-preliminary information vs. no forewarning-control). This analysis showed a main effect of forewarning, $F(2,111)=3.67, p<.05, \eta^{2}=.06$. Post-hoc LSD comparisons indicated that participants forewarned of an influence attempt complied less with the request to clear up their lecture-halls $(M=32.63, S D=54.76)$ than participants in the no forewarningcontrol condition $(M=70.13, S D=69.06), p<.01, d=.60$. The other two comparisons were not significant; participants who were not forewarned and received preliminary information $(M=50.68, S D=65.32)$ were neither statistically different from forewarned participants, $p=.20, n s$, nor from non-forewarned-control participants, $p=.17$, ns. No main effect of self-regulatory resource depletion condition was found, $F=1.00, n s$.

Of main importance for our hypothesis, the ANOVA showed the expected interaction effect between self-regulatory resource depletion condition and forewarning, $F(2,111)$ $=3.69, p<.05, \eta^{2}=.06$. In line with the results of Experiment 4.1, additional simple main 
effects analyses showed that forewarning of an influence attempt increased resistance to compliance among depleted individuals, $F(2,111)=6.82, p<.01$. As shown in Table 4.3, when depleted, forewarned participants complied far less with the request to voluntarily clear up their lecture halls as compared to their non-forewarned counterparts $(p<.01$, $d=1.12$ ), and non-forewarned participants who received preliminary information, $p<$ $.05, d=.89$. For the two depleted groups that did not receive a forewarning, receiving preliminary information did not have an effect on compliance, $p=.38$, ns. Similar to the results of Experiment 1, for non-depleted participants the effect of forewarning did not reach significance, $F<1$.

Table 4.3

Average number of minutes participants volunteered as a function of resource depletion and forewarning

\begin{tabular}{lcccccc}
\hline & \multicolumn{2}{c}{ Forewarning } & \multicolumn{2}{c}{ Preliminary Information } & \multicolumn{2}{c}{ No Forewarning } \\
\hline & $M$ & $S D$ & $M$ & $S D$ & $M$ & $S D$ \\
\hline Depletion & $16.50 a$ & 22.25 & $68.33 b$ & 79.08 & $86.25 b$ & 84.84 \\
\hline No Depletion & $48.75 a$ & 71.50 & $33.95 a$ & 44.96 & $54.00 a$ & 45.24 \\
\hline
\end{tabular}

Note. Means in the same row that do not share subscripts differ at $\mathrm{p}<.05$.

Together, these results replicate and extend the findings of Experiment 4.1. In line with our key hypothesis, we found that when self-regulatory energy is low, a forewarning of an influence attempt motivates people to conserve their remaining resources for future resistance. Consequently, these individuals perform worse than participants in all other conditions on an intervening measure of self-control (cf. Muraven et al., 2006). Our results also support the notion that conservation of self-regulatory energy is not the product of merely receiving preliminary information about an upcoming encounter; only when this information is accompanied by a forewarning of this encounter entailing an influence attempt, are people concerned with conserving strength to be able to offer resistance. When subsequently confronted with a request, initially depleted participants 
who conserved their resources resist as much as non-depleted participants. Furthermore, this beneficial effect of conservation creates an inevitable contrast with depleted individuals who did not anticipate exerting self-control in the future. More specifically, depleted participants who were not forewarned of an impending influence attempt resisted less than all other participants (who were either not depleted, or depleted but had conserved resources), presumably because they spent all their strength on the initial two tasks, leaving them with the lowest amount of resources of all six groups to resist the subsequent request for compliance (cf. Muraven et al., 2006).

In line with attitude change research (e.g., Petty \& Cacioppo, 1977), we assumed that forewarning people of an impending influence attempt would increase resistance to persuasion, because it encourages more systematic processing of message-relevant information and stimulates the generation of counterarguments. The previous two studies focused on the outcome of this presumed process, which was resistance to influence as measured by the amount of (non)compliance with a persuasive request. Therefore, instead of using compliance as an outcome measure, in a third study we directly addressed the process of counterargumentation as an indicator of resistance to influence. More specifically, we tested whether people who were initially depleted, but conserved their resources due to a forewarning, would generate more arguments in opposition to a persuasive request than depleted individuals who did not receive a forewarning. Since there would be no direct need to conserve resources when one has not previously performed an act of selfcontrol, we did not expect this effect of forewarning among non-depleted participants.

In addition, to generalize the results beyond the specific research settings employed in the previous studies, we used alternate means of inducing a state of self-regulatory resource depletion and forewarning people of an impending influence attempt. We also used a different self-control task to measure whether people conserved their resources for future resistance. 


\section{Experiment 4.3}

\section{Design and procedure}

Seventy-nine undergraduate students (57 female, 22 male; mean age 19.76 years, SD $=2.79$ ) participated in exchange for partial course credit. The study used a 2 (selfregulatory resource depletion condition: depletion vs. no depletion) X 2 (forewarning: forewarning of an influence attempt vs. no forewarning) between-subjects factorial design.

On arrival at the laboratory, participants were seated in individual cubicles fitted with a desktop computer, which presented all the instructions. Participants were randomly assigned to one of the four conditions, and informed that the experiment consisted of several different, unrelated tasks.

To induce a state of self-regulatory resource depletion, we had participants control their speech (cf. Muraven \& Slessareva, 2003). All participants were instructed to improvise a three-minute story about themselves, using a voice recorder which was placed in their cubicle to record their speech. The computer indicated when to start and when three minutes had passed. Participants in the no depletion control condition were allowed to speak freely, while participants in the depletion condition were instructed to avoid using the filler Um and the word I. Applying such a rule requires self-control, because one has to actively override the habit to use the filler Um in (improvised) spoken language, and the word I in an autobiographical story.

All participants then read on their computer screen that the next task would be measuring 'mental speed'. Additionally, only participants in the forewarning condition were informed about an upcoming influence attempt: 'We would like to call to your attention that after this test the experimenter would like to give you some information 
about the 'Orphans and Vulnerable Children (OVC) Sponsorship Program' that a colleague of hers works for. She is actively looking for new sponsors willing to financially contribute to this project.'

\section{Dependent measures}

Self-regulatory resource depletion. To measure whether participants would conserve their self-control resources when forewarned about an upcoming influence attempt, we asked them to perform another self-control task, which was introduced as measuring 'mental speed'. All participants were asked to solve 32 trials of a cognitive test, which entailed indicating whether a mathematical equation was true or false (e.g., 100/10 = 25), or whether a picture and a word that were shown as a pair had the same denotation (e.g., they saw the word 'clock' together with a picture of a cow). After every trial they had to indicate as quickly as possible whether it was true or false, but randomly the word 'reverse' appeared on screen, which meant that they had to reverse their answer (true became false and vice versa). We expected that it would be harder for participants low in self-control, or conserving resources, to override the initial dominant response to provide the correct answer on the reversed trials, resulting in larger response-latencies. Hence, average response times served as our key dependent variable to assess selfcontrol performance.

Counterargumentation. Next, all participants were provided with information about the Orphans and Vulnerable Children (OVC) Sponsorship Program, which is an existing charity program that financially supports educational facilities for children in Cameroon (http://www.navtifoundation.org/orphanvol-children.htm). All participants were asked what arguments they could list against financially supporting this program, no matter whether they would personally like to donate money to this charity or not. Afterwards, all participants were debriefed and thanked. 


\section{Results and discussion}

Self-regulatory resource depletion. An ANOVA was conducted on participants' mean response-time on the 32 trials of the cognitive test, as a function of self-regulatory resource depletion condition (depletion vs. no depletion) and forewarning (forewarning of an influence attempt vs. no forewarning). Incorrect responses were recoded as missing. The ANOVA showed a main effect of both factors. Participants who had to control their speech took significantly longer to respond to the trials of the cognitive test $(M=3.36, S D=.68)$ than participants who were allowed to speak freely $(M=3.13, S D=.55)$, indicating that they were more depleted than participants in the control-condition, $F(1,75)=5.22, p<.05, d=.37$. Furthermore, participants forewarned about the upcoming influence attempt responded slower to the trials of the cognitive test $(M=3.37, S D=.68)$ as compared to participants who were not forewarned $(M=3.12, S D=.54), F(1,75)=6.06, p<.05, d=.41$

Most important, the analysis also showed the predicted interaction effect between selfregulatory resource depletion and forewarning, $F(1,75)=5.79, p<.05, \eta^{2}=.07$. Similar to Experiment 4.2, we expected depleted participants who were forewarned of an upcoming influence attempt to maximally conserve their remaining self-control resources as compared to participants in the other conditions, who were either not forewarned or not initially depleted. Therefore, we expected depleted and forewarned participants to show the poorest performance on the cognitive test since they would be most concerned with conserving their remaining resources to resist the upcoming influence attempt. Hence, we predicted a significant contrast between the depletion-forewarning condition and the remaining three conditions that were not expected to differ significantly from each other. Following the procedure outlined by Bobko (1986), the first contrast tested the equality of the conditions whose means are assumed to be equivalent, using a one-way analysis of variance. As expected, this analysis showed the means of the depletion-no forewarning, no depletion-forewarning, and no depletion-no forewarning conditions to be statistically equivalent, $F<1$, ns. Hence, these three groups performed similarly on the cognitive test, 
indicating that there was no differential tendency to conserve self-regulatory resources. The second contrast tested whether depleted participants anticipating an influence attempt performed worse on the cognitive test than the average of participants in the other three conditions, using a planned comparison t-test. This analysis confirmed our prediction that depleted and forewarned participants conserved their self-control strength to a higher extent than participants in any of the other conditions, as evidenced by a slower average response-time, $t(75)=21.11, p<.001$. Furthermore, a series of simple contrast analyses, with the "depletion-forewarning condition" as the referent category, confirmed that participants in this condition indeed performed worse on the cognitive test: on average they responded slower $(M=3.76, S D=.69)$ than participants in the depletion-no forewarning condition ( $M$ $=3.11, S D=.54, t(75)=3.27, p<.01, d=1.05)$, the no depletion-forewarning condition $(M=$ $3.14, S D=.57, t(75)=3.17, p<.01, d=.98)$, and the no depletion-no forewarning condition $(M=3.13, S D=.54, t(75)=3.12, p<.01, d=1.02)$.

Counterargumentation. An ANOVA was conducted on the number of arguments that participants generated against donating money to the OVC charity program, as a function of self-regulatory resource depletion condition (depletion vs. no depletion) and forewarning (forewarning of an influence attempt vs. no forewarning). This analysis showed no main effects of self-regulatory resource depletion $(F=1.31, n s)$ and forewarning $(F<1, n s)$, but did show the expected interaction effect between these two factors, $F(1,75)=6.49, p<.05$, $\eta^{2}=.08$. Additional simple main effects analyses showed that forewarning of an influence attempt increased resistance among depleted individuals, $F(1,75)=5.44, p<.01, d=.72$. When depleted, forewarned participants appeared to generate more counterarguments $(M=2.86, S D=1.35)$ as compared to their non-forewarned counterparts $(M=2.09, S D=$ .68). For non-depleted participants the effect of forewarning did not reach significance $\left(M_{\text {Forewarning }}=2.04, S D=.88, M_{\text {No forewarning }}=2.40, S D=1.00\right), F=1.47, n s$. In accordance with the results of Experiments 4.1 and 4.2, forewarning of an impending influence attempt increased resistance among initially depleted individuals; they generated even more counterarguments than participants that were not initially depleted. 


\section{General discussion}

Although previous research has shown that a state of self-regulatory resource depletion makes people more susceptible to influence (e.g., Fennis et al., 2009; Wheeler et al., 2007), the present research demonstrates that, under certain conditions, depleted individuals can successfully conserve their resources to be put into action when subsequently confronted with an influence attempt. Three studies showed that forewarning of an impending influence attempt increases resistance to persuasion among depleted individuals: anticipation of a persuasive request decreased their amount of compliance and increased the number of arguments they generated against it. Moreover, the present studies provide support for the assumed underlying psychological process: forewarning directly affects self-regulatory resources in that it appears to motivate depleted individuals to conserve their remaining self-control strength to ward off the subsequent influence attempt. The results of Experiment 4.2 also support the notion that conservation of self-regulatory energy is not the product of merely receiving preliminary information about an upcoming encounter. Only when this information is accompanied by a forewarning that this encounter has a persuasive intent, are people concerned with conserving their self-control resources to be able to resist the upcoming persuasive appeal. This corresponds with the findings of Muraven et al. (2006), which show that the motivation to conserve was not affected by merely anticipating a forthcoming task but by the fact that this future task required exerting self-control.

In line with the work of Muraven et al. (2006), the present research shows that having exerted self-control in the past and expecting to exert self-control in the near future motivates and enables people to conserve their self-regulatory strength. When it comes to this future task, conservation of resources cancels out the detrimental effect of initial depletion, creating an inevitable contrast with depleted individuals who did not anticipate exerting self-control in the future. In the present studies depleted individuals who conserved resources due to a forewarning were more resistant to persuasion than 
depleted individuals who were not prompted to be efficient in their use of self-control energy. Indeed, additional contrast analyses on the dependent measure of compliance in Experiments 4.1 and 4.2 confirm that depleted participants who were not forewarned of an impending influence attempt complied more and thus resisted less than the average of all other participants (who were either not depleted, or depleted but having conserved resources) ${ }^{1}$. Moreover, contrast analyses confirmed that participants, who were initially depleted but conserved their resources due to a forewarning, were as much resistant to influence as participants who were not initially depleted ${ }^{2}$. In Experiment 4.3 we assessed the process of resistance to persuasion more directly: instead of measuring the amount of (non)compliance we asked participants to counterargue a persuasive request. In line with the results of Experiments 4.1 and 4.2, this study showed that forewarning of an influence attempt increased resistance to persuasion among depleted individuals; anticipating a persuasive request motivated depleted individuals to conserve self-regulatory resources, and stimulated the generation of counterarguments. Interestingly, the pattern of results of Experiment 4.3 also suggests that for non-depleted participants the effect of forewarning on counterargumentation reverses, with forewarned participants producing fewer counterarguments than nonforewarned participants. However, we should note that this difference is spurious as the contrast failed to reach significance. Nevertheless, future research might address this issue in more detail.

Corroborating previous research (e.g., Muraven et al., 2006; Muraven \& Slessareva, 2003), the current research shows that external motivators can encourage individuals to overcome depletion by carefully allocating their remaining self-control strength, which raises questions about the limited nature of self-control. In line with Muraven et al. (2006), the present findings suggest that a decline in self-control performance after a previous act of self-regulation may reflect either a lack of ability (as in the 'classic' ego-depletion studies, e.g., Baumeister et al., 1998), or a lack of motivation in that people become less willing to invest the required resources to succeed at self-control, 
simply because it is deemed too costly and because they are more concerned with conserving strength for future self-control purposes. In this respect, it is important to note that in the present studies a forewarning only affected self-control performance of depleted individuals. If one's resources had not previously been depleted, there was no direct need for conservation, since one would have enough resources for resisting a future influence attempt. As Muraven et al. (2006) already remarked, non-depleted people likely need a very good reason to conserve, as they are less sensitive to future demands than depleted individuals. Maybe if we used a more powerful forewarning (such as the expectation to be the target of influence of a highly professional influence agent), non-depleted participants might also have conserved strength. In general, it becomes increasingly clear that self-regulation involves a constant (unconscious) trade-off between multiple self-control demands, and the current research confirms that the ability and the motivation to self-regulate are strongly related. For future research, it would be fruitful to try to differentiate and explore the interplay of these processes, as to enlighten the inner workings of self-control further.

With respect to the forewarning literature, the present research has shown that a forewarning of influence is not only an effective tool for increasing resistance to persuasion in the domain of attitude change, but also in the domain of behavioral change processes. Participants complied significantly less with a persuasive request when they were warned about the upcoming influence attempt in advance. In line with attitude change research (e.g., Petty \& Cacioppo, 1977), Experiment 4.3 shows that forewarning people of an impending influence attempt encourages more systematic processing and stimulates the generation of counterarguments. Importantly, the present studies now provide insight in the self-regulatory dynamics underlying these effects of forewarning on persuasion. To resist persuasion, people need self-control strength to counterargue a persuasive request or message, and when these resources are not optimal, a forewarning promotes self-regulatory efficiency and prompts those low in self-control strength to conserve their remaining resources. Thus, a forewarning of persuasion is especially 
beneficial to those low in self-control strength, so that they can build up their resistance. In that respect, our studies are the first to show that the effect of forewarning is not just a matter of increasing the motivation to resist persuasion; the effect of forewarning depends on the amount of regulatory resources a person has available, given that it is more pronounced when people's resources are diminished. Eventually, there will be no difference in the extent of resistance between people who were depleted but conserved resources, and people who had their resources available from the beginning. Whether these processes can be consciously controlled, or whether the process of conserving resources for subsequent counterargumentation and resistance to persuasion operates mainly beyond our conscious awareness, still remains to be tested.

The present studies underscore previous research which has shown that resistance to persuasion is an effortful activity which depends on one's self-regulatory capacities. People need self-control resources to actively resist a persuasion attempt, to be able to scrutinize a message or request and argue against the persuasive communication. Being low in self-control strength weakens our defense, and when one is not prompted to do something about it, as in conserving and mobilizing remaining strength, one's attempts at resistance are more likely to fail. Gathering knowledge on and insight into resistance processes is relevant to a host of influence contexts, yet the issue is probably most germane to the field of marketing and consumer behavior. In contrast to such non-profit domains as health promotion or risk communication, where influence attempts are sometimes welcomed by the message recipient, commercial influence is typically met with a less accepting, more skeptical response. Indeed in the marketing sphere, unsolicited influence appears to be the rule rather than the exception. People are frequently being harassed by telemarketers after a day of hard work, sales representatives and fundraisers approach shoppers with unwanted offers, and television programming is frequently interrupted by unwanted advertising messages. It seems that the quantity of persuasive messages and requests we encounter is growing by the day, and often it is not even what these want to persuade us about, but it is the fact that 
we are targets of influence even in the privacy of our homes that makes it unwanted and makes us want to protect our personal freedom. Fortunately, the present results have shown that a temporary lapse in self-control ability does not necessarily result in involuntary acquiescence to the wishes of these influence professionals. To the extent that depleted individuals anticipate upcoming influence attempts, they remain able to resist unsolicited persuasion.

Although the forewarning itself may or may not accompany an influence attempt in commercial consumer settings, advanced awareness of the upcoming persuasion attempt may well be a default mode for people when they enter marketing spheres such as shopping malls or commercial websites. In addition to forewarnings being explicitly present, it seems likely that they are often self-generated, based on people's previous experiences with commercial settings: in a shopping mall the sales representatives can be spotted miles away, practically every television viewer will be aware of the interruptions by persuasive messages, and magazine ads are often explicitly labeled as 'advertisement' to distinguish them from the journalistic content. Indeed, work on the persuasion knowledge model (Friestad \& Wright, 1994) supports the notion that consumers are typically prone to infer the (self-serving) motives of marketers and advertisers. People are becoming increasingly aware of the numerous commercial traps they can fall into, and this knowledge could function as a source of self-generated forewarnings. Hence, we should be comforted by the present results which imply that we are still proficient in defending ourselves, despite a general increase in susceptibility to influence. Even in these instances we will not always end up subscribing to a cause we do not fully support, or end up with products and services that we do not want or need. 


\section{Footnotes}

1. We performed a planned comparison t-test on the dependent measure of compliance in Experiment 4.1, weighting the depletion-no forewarning condition +3 , and the depletion-forewarning, the no depletion-forewarning, and the no depletion-no forewarning each -1. This analysis supported our prediction, $t(109)=5.11, p<.001$. For Experiment 4.2, we weighted the depletion-no forewarning-preliminary information and the depletionno forewarning-control conditions each +2 , and the depletion-forewarning, the no depletion-forewarning, no depletion-no forewarning-preliminary information, and no depletion-no forewarning-control conditions each -1 . This analysis also supported our prediction, $t(111)=3.19, p<.01$.

2. We tested the equality of the three conditions of Experiment 4.1 whose means are assumed to be equal (depletion-forewarning, no depletionforewarning, no depletion-no forewarning) using a one-way ANOVA, which indeed showed the means to be statistically equivalent, $F(2,88)=2.33$, $n$ s. We also tested the equality of the four conditions of Experiment 4.2 whose means are assumed to be equal (depletion-forewarning, no depletionforewarning, no depletion-no forewarning-preliminary information, and no depletion-no forewarning-control). Again, a one-way ANOVA showed the means to be statistically equivalent, $F(4,96)=1.71, n$. 




\section{Chapter 5}

General discussion 

$\mathrm{n}$ intriguing question, which has become an important topic of study within
the field of marketing communication and consumer psychology, is what makes consumers comply with (unsolicited) requests. Why do people accept offers they were not planning to yield to in advance, investing time, effort, or money supporting causes and organizations they may have never heard of before, often without expecting a return on their investment? What makes it so hard for consumers to resist the influence attempts targeted by salespeople, fundraisers, and other influence professionals? And in contrast, what determines whether people succeed at resisting persuasion? The research presented in this dissertation approached these questions from a selfregulation perspective, suggesting that people's failure and success in dealing with (unwanted) persuasion depend for an important part on the availability of resources to actively control the self. By addressing the ability and motivational aspects of self-control and focusing on the role of self-regulation in both compliance as well as resistance in response to persuasive requests, this dissertation aimed to advance our understanding of the dynamics behind resisting and yielding in social influence situations that people encounter each day.

In this final chapter, a summary of the main empirical findings that were reported in this dissertation will be presented. This will be prefaced by a short review of the introductory chapter, where a two-stage model was introduced providing the basic reasoning of the research presented in this dissertation. After the overview of the main findings, the theoretical implications and contributions of the empirical findings will be discussed, as well as some limitations of the present studies and potential directions for future research. This chapter will conclude with the practical implications of the present research, that will be considered from the side of the influence professional, as well as from a consumer point of view. 


\section{Summary of the empirical findings}

To study the role of self-regulation in social influence contexts, the research presented in this dissertation concentrated on a common means for influence professionals to elicit consumer compliance: scripted social influence strategies. Decades of studies on social influence confirm that consumers are induced to comply with a request at much higher rates when approached with a social influence technique than when the request is made without a scripted warm-up period (Burger, 1999; Cialdini \& Goldstein 2004). Among a large variety of strategies that have been reported in the literature are the foot-in-the-door technique (Freedman \& Fraser, 1966) and the door-in-the-face technique (Cialdini et al., 1975).

To explain the effectiveness of these techniques, the principle of automaticity has been forwarded as a key determinant (Cialdini, 1993; Cialdini \& Goldstein, 2004). Instead of carefully scrutinizing the merits of a request or offer, people appear to respond mindlessly (i.e., with little conscious effort) when confronted with a social influence technique. Under these conditions of reduced mental alertness, people are thought to fall back on habit and routine and are prone to employ 'mental shortcuts' or heuristics in their judgment and decision making. Use of these heuristics will generally increase the likelihood of compliance (Cialdini, 1993).

The question that was key to the research in this dissertation is what produces the mindlessness in these influence contexts. Why do people proceed with a minimum of cognitive effort or thought and behave automatically, falling back on heuristics for decision making, when confronted with an influence technique? It was proposed that the origins of this mindlessness could be found in a characteristic that almost all successful influence techniques have in common: multiple decision moments or sequential requests (Fern et al., 1986). The target of influence has to yield to one or several initial request(s), answer probing questions, or making choices before the target 
request is presented. In line with the assumptions of the limited-resource model of selfcontrol (Baumeister, Vohs, \& Tice, 2007), it was proposed that the preliminary stage of these sequential request techniques triggers one underlying psychological mechanism that accounts for the impact on subsequent compliance: self-regulation failure brought about by self-regulatory resource depletion (Baumeister et al., 1998; Vohs \& Heatherton, 2000; for a review, see Baumeister, Vohs, \& Tice, 2007). Like any other behavior that involves deliberate and regulated responses by the self, it was argued that consciously attending and actively responding to the initial requests of an influence attempt draws on a limited intrapsychic resource, akin to strength or energy. In a subsequent state of self-regulatory resource depletion, the controlled, purposeful self fails to function effectively, which renders people vulnerable to untoward impulses, habit, routine, and automatic processes (Baumeister \& Vohs, 2007; Vohs et al., 2005), all key indicators of mindlessness.

The reasoning as stated above was represented in a two-stage model (see Figure 1, Chapter 1), presenting a self-regulatory resource depletion account of the impact of sequential request techniques. Specifically, this model claims that yielding to the initial request-phase of an influence attempt taxes consumers' self-control resources. Enhancing the weight on automatic processing, a lowered level of self-regulatory resources then fosters the use of heuristics, when present in the persuasion context, which increases the odds of yielding to the target request of the influence technique. In Chapters 2 and 3, this two-stage model was elaborated and tested in a series of eight studies, conducted in the laboratory as well as in more naturalistic settings. In Chapter 4, empirical evidence from three studies was presented that extended the claims made by the two-stage model. Focus was shifted to the motivational aspects of self-regulation and self-regulatory efficiency was uncovered as a successful (unconscious) defense strategy against an upcoming persuasive attack, for individuals low in self-control. The specific outcomes of the experiments presented in each chapter are presented below. 
Chapter 2. Stage one: Weakening the ramparts: Actively responding to an influence attempt induces self-regulatory resource depletion

Chapter 2 focused on the first stage of the two-stage model. It was proposed that during the initial phase of a social influence procedure consisting of multiple requests, a state of self-regulatory resource depletion would be induced. People's self-control resources are likely to be worn down during this phase, because one has to consciously attend and actively respond to one or several initial questions or requests. Responding to these requests is often highly involving, since they entail either active self-presentation or demanding cognitive operations, or both - processes that are known to elicit selfregulatory resource depletion (Schmeichel et al., 2003; Vohs et al., 2005).

Four studies tested the hypothesis that yielding to an initial request to answer a series of self-presentational or cognitively demanding questions induces self-regulatory resource depletion. The first three studies were conducted in a field setting. Experiment 2.1 showed that answering a series of 11 open-ended questions about personal health behavior and lifestyle (presumably fostering impression management motives) increased participants' scores on the State Ego Depletion Scale (Ciarocco et al., 2010), thus indicating that they were more depleted than participants who were not requested to answer these questions.

Experiments 2.2 and 2.3 sought to extend the results of Experiment 2.1 in several important ways. Similar to Experiment 2.1, participants in the initial request condition were asked extensively about their personal health behavior and lifestyle, but participants in the control condition were now also involved in an unanticipated interpersonal interaction with an unknown person, which lasted the same amount of time. Experiment 2.2 used a validated self-control task to assess resource depletion: participants who answered the series of self-presentational questions persisted less on a subsequent figure tracing puzzle than participants in the control condition. By 
using multiple requesters, Experiment 2.3 ruled out the alternative explanation that violating the norm of reciprocity accounted for the earlier findings: after answering the taxing initial questions participants did not experience increased resistance to further accommodate the requester, when compared to the control condition. Moreover, this experiment confirmed that responding to the initial questions influenced active impression management concerns, but did not (negatively) affect emotions or liking for the confederate. Converging evidence was found in two other studies that were not reported in this dissertation (Fennis et al., 2009, Experiments 1 and 2).

The previous studies demonstrated self-regulatory resource depletion as an outcome of responding to the initial stage of a social influence technique designed to promote compliance. Yet the two stage model posits that regulatory resource depletion functions as a mediating variable, produced by the initial stage of a compliance gaining technique and, in turn, fostering compliance with the target request. Experiment 2.4, which was conducted in a laboratory setting instead of a field setting, sought to bridge Stage 1 and Stage 2 of the model by directly assessing this mediating role. Moreover, instead of manipulating self-presentational demands as used in the previous three experiments, this study included an initial request which required answering a series of cognitively demanding questions. This condition was contrasted with an initial request with an equal number of questions of a less demanding nature. The study showed that the impact of answering ten challenging questions about the Dutch Tax and Customs Administration on people's willingness to volunteer as a future research participant in their studies, was mediated by self-regulatory resource depletion (as measured by the average reaction time on a Stroop task [Stroop, 1935] ). A similar mediational effect of self-regulatory resource depletion was found in a study by Fennis and Janssen (under review).

Taken together, these four studies provided support for Stage 1 of the model, in showing that yielding to an initial request of a sequential request procedure (answering a series of self-presentational or cognitively demanding questions) induces self-regulatory 
resource depletion. Moreover, Experiment 2.4 linked Stage 1 of the model to Stage 2, in showing that self-regulatory resource depletion fully mediates the effect of responding to an initial request on compliance with the target request of a sequential request procedure. By demonstrating this mediational effect, this study extends the other work in this dissertation, by providing a comprehensive account of the role of self-regulatory resource depletion in the effectiveness of social influence techniques. Stage 2 of the model was further elaborated in Chapter 3.

\section{Chapter 3. Stage two: The path of least resistance: Self-regulatory resource depletion affects compliance through heuristic decision making}

As a direct extension of the previous chapter, Chapter 3 focused on the second stage of the two-stage model. It was proposed that self-regulatory resource depletion would reduce systematic or central-route processing, enhancing the weight on heuristic processing in consumer judgment and decision making (cf. Wheeler et al., 2007). When people are subsequently presented with a request, compliance will be heightened to the extent that a suitable heuristic is present in the influence context, which points to compliance as an efficient behavioral outcome.

Four studies tested the hypothesis that a state of self-regulatory resource depletion heightens compliance through reliance on heuristics. In Experiment 3.1, participants whose self-regulatory resources had been lowered (by a task adopted from Baumeister et al. [1998] which requires inhibiting an overlearned response) were willing to volunteer for a larger amount of time as an experimenter in future studies, as compared to participants whose resources had been untouched. However, self-regulatory resource depletion per se did not result in enhanced compliance, but only when a compliance-promoting heuristic was part of the influence setting, providing a basis for decision-making. The heuristic principle featured in this study was the principle of reciprocity, i.e. the felt obligation to return favors (Gouldner, 1960). The salience of this heuristic was manipulated by excusing 
participants of a boring part of the experiment, inducing a counterconcession on the part of the participant (Cialdini, 1993). Experiments 3.2 and 3.3 replicated the results of Experiment 3.1, using different manipulations of self-regulatory resource depletion and heuristic activation, and measuring different forms of compliance. Specifically, Experiment 3.2 showed that participants who had to control their attention during a video (a selfcontrol task adopted from Schmeichel et al., 2003) donated a larger percentage of money to a charity than participants who did not have to control their attention. However, this was only the case when the charity was a well-known organization, described as renowned and experienced (thus activating the heuristic principle of authority, Cialdini, 1993). Similarly, Experiment 3.3 showed that the heuristic principle of likeability, which was activated by providing participants with a compliment on their task performance, moderated the effect of self-regulatory resource depletion on compliance with a request to volunteer as a participant in future studies of a research society. Resource depletion (as manipulated with a mirror-tracing persistence task adopted from Quinn et al., 1996) only increased compliance rates when the likeability principle was activated. Moreover, this study was conducted in a more naturalistic setting, rather than in the laboratory.

Experiment 3.4 sought to extend the results of the previous three studies by assessing individual differences in dispositional self-control (Tangney et al., 2004). To the extent that the proposed model is general, we expected to see not only that low self-regulatory resources can be induced by situational demands but also that people dispositionally low in self-control respond in a similar fashion. The heuristic principle that was made salient was the principle of reciprocity, that was also featured in Experiment 3.1, using a different manipulation in the current study: participants were done a favor by their university, inducing a counter-concession on their part. Participants low in dispositional self-control appeared to be particularly susceptible to this heuristic, resulting in increased compliance with a request, which in this experiment was the willingness to do unpaid work for their university. Converging evidence was found in a study by Fennis et al. (2009, Experiment 6), demonstrating that the low-ball technique (a social influence strategy which features 
the heuristic principle of commitment) was particularly effective among people low in dispositional self-control.

Together, these four studies provided support for Stage 2 of the model, in showing that a reduced supply of self-regulatory resources fosters compliance with a target request, but not by default. Rather, it was shown to do so through an overreliance on salient heuristics that facilitated compliance as an efficient behavioral response. In sum, the results of the empirical studies as presented in Chapters 2 and 3 of this dissertation offer an explanation for the mindlessness so often observed in social influence situations. Self-regulatory resource depletion (Baumeister, Vohs, \& Tice, 2007) appears to be an important underlying psychological mechanism that accounts for the impact of social influence techniques. When self-regulatory resources have been lowered during the initial stage of a sequential request procedure, people more easily resort to salient heuristics for decision making, thereby increasing the chance that they will comply with a request to invest time, effort, or money. The research presented in Chapter 4 provided an extension of the model, in focusing on the role of motivational aspects of self-control in resisting social influence attempts.

\section{Chapter 4. Extending the model: Forewarned is forearmed: Conserving self-control strength to resist social influence}

In Chapter 4, empirical evidence was presented that extends the claims made by the twostage model. The demonstration that self-regulation failure may be at the base of yielding to compliance implies that successfully resisting persuasion will also depend for an important part on the availability of resources to actively control the self. Hence, whereas a low level of self-control resources increases susceptibility to influence, a high level of regulatory resources likely increases the chance that one is able to resist an influence attempt. But does a temporary lowered level of self-regulatory resources automatically imply a weakened defense against an influence attempt? The role of motivation was argued to be key here. 
The research in Chapter 4 proposed that individuals low in self-control resources can still be successful at defending themselves against a persuasive attack, when prompted to be efficient in allocating their remaining self-regulatory resources. Based on the notion that a depleted state does not reflect a complete exhaustion of resources but merely a temporary or relative deficit (Muraven et al., 2006), it was proposed that initially depleted people can still be successful at resisting persuasion when they temporarily economize on their use of self-control resources, and avoid spending more strength. Specifically, a forewarning of an upcoming influence attempt should motivate these individuals to conserve their remaining resources (although this comes at a cost, and self-control will temporarily suffer), enabling them to be just as successful at resisting persuasion as nondepleted participants. However, a forewarning was expected to function as a motivational factor only for people who previously suffered a loss of self-control resources, otherwise there would be no need to conserve.

Three studies tested the hypothesis that individuals low in self-control resources show more resistance in response to a persuasive request when given a forewarning of an upcoming influence attempt. In Experiment 4.1, participants whose self-regulatory resources had been lowered (using a similar task as in Experiment 3.1) were willing to volunteer for a smaller amount of time for a student project when they were forewarned about an upcoming persuasive encounter with a representative of this project. As expected, non-depleted individuals were not affected by the forewarning.

A second study was performed to uncover whether the assumed underlying psychological process driving this effect was indeed conserving self-control strength. To test this notion, Experiment 4.2 included a validated measure of self-control performance after the depletion and forewarning manipulations, preceding the compliance measure. The design and procedure paralleled that of study 4.1. As expected, results showed that a forewarning of an impending influence attempt motivated depleted participants to conserve their resources: they performed worse than participants in any other condition 
on the intermediate measure of self-control (cf. Muraven et al., 2006). As in Experiment 4.1, when subsequently confronted with a request, initially depleted participants who were forewarned resisted as much as non-depleted participants, in contrast to initially depleted participants who were not forewarned and thus did not conserve their resources. Moreover, by adding a second control condition in which participants were not forewarned about an influence attempt but did receive preliminary information about the upcoming encounter, the results of this study confirmed that the forewarning is solely responsible for the effect.

In extension to the previous two studies that assessed the amount of noncompliance as an indicator of resistance to influence, Experiment 4.3 directly addressed the process of counterargumentation in response to a persuasive request. Using alternate means of manipulating a state of self-regulatory resource depletion and forewarning and a different measurement of conservation of resources, this study replicated the previous results. Initially depleted individuals who were forewarned of an upcoming influence attempt generated more arguments against a persuasive request than initially depleted participants who were not forewarned, because they conserved their resources in advance.

Whereas the research presented in Chapters 2 and 3 of this dissertation demonstrated that self-regulatory failure increases the chance that one yields to compliance, the results of the three studies presented in Chapter 4 have shown that all is not lost for those in a state of self-regulatory resource depletion. A forewarning appears to motivate depleted individuals to conserve their resources to be put into action when subsequently confronted with an influence attempt. By prompting self-regulatory efficiency, a forewarning enabled them to be just as successful at resisting an influence attempt as individuals whose resources had been untouched. These studies underscore the joint roles of ability and motivation in shaping self-regulatory processes in social influence and persuasion. 


\section{Discussion of the empirical findings: Theoretical implications and contributions}

The research presented in this dissertation pointed out self-regulatory resource depletion as a key mechanism responsible for the effectiveness of many social influence situations, thereby contributing to our knowledge in several ways. By connecting two broad fields of research, that of self-regulation as a limited resource, and that of social influence processes, it advances our understanding in both domains.

Although a self-regulatory framework has recently been put forward in studies on attitude change processes (Burkley, 2008; Wheeler et al., 2007), the role of self-regulation has until now been neglected in studies on behavioral compliance. In this respect, the present research offers a new way of looking at the processes by which resistance and yielding to influence operate. First and foremost, the present research offers an explanation for the mindlessness so often observed in social influence situations. Specifically, automaticity has been proffered as a basic requirement for a variety of social influence techniques to work, but no study to date has directly addressed this key question. By studying the role of self-regulation in social influence situations, we have been able to point out self-regulatory resource depletion as an important underlying factor that could explain why people so often fail to say "No" to an influence agent, and end up with a deal that does not necessarily benefit them. Moreover, by gaining these insights we were also able to enlighten under which circumstances people do succeed at defending themselves against an (unsolicited) influence attempt. The studies presented in Chapter 4 of this dissertation point to resistance to persuasion as a reflective, effortful process, that gets impaired when resources for self-regulation are

low (as evidenced by a reduced amount of counterargumentation in Experiment 4.3 of this dissertation). However, Chapter 4 shows that when resources are low, a motivation to engage in self-regulatory efficiency proves to be a successful (unconscious) strategy to compensate for a lack of resources, and successfully resist a persuasive appeal. 
Furthermore, by linking up self-control ability with 'classic' dual-process models of persuasion, the present research underscores the self-regulatory mechanisms driving systematic or central information processing in response to persuasion attempts. Actively resisting persuasion most likely requires considerable cognitive effort and more thorough information processing for people to be able to engage in issue relevant thinking and generate arguments against an influence attempt. A state of self-regulatory resource depletion interferes with people's abilities to critically evaluate persuasive argumentation, and enhances the weight on heuristic processing, thereby increasing the odds of compliance with a persuasive request, provided that there is a suitable heuristic present in the persuasion context.

In this respect, it is important to recall that recent views on self-regulation as a limited resource emphasized that a depleted state does not necessarily imply that people's resources for self-regulation are completely exhausted (Muraven et al., 2006). Rather, it is a matter of a temporary or relative deficit, which increases the value of the resources that remain and decreases people's willingness to exert them. A state of self-regulatory resource depletion can therefore not simply be described as a loss of resources, but also contains a motivational aspect, in that people in such a state are more concerned with conserving strength. Adopting such a functional or economic perspective, we could state that in a depleted state, people will only let their remaining resources suffer when the need to self-regulate at that point predominates their concerns for self-regulation later on. Relying on more associative, heuristic processing has the economic advantage of requiring a minimum of cognitive effort, and will therefore be a common strategy when people are less able and therefore less willing to exert self-control energy. In our studies, depleted participants were possibly not sufficiently motivated to work against an agent of influence because they were confronted with relatively mild requests. When an easily accessible heuristic was provided, detailed processing of the content of the persuasive request could be avoided and compliance was the least taxing way out of the encounter. However, things could be different when a request becomes too 
large. Compliance with a relatively large request will be more consequential and likely heightens the motivation for in-depth information processing (cf. Pollock et al., 1998). It is therefore plausible that in such a situation people will overcome their depleted state, and mobilize the resources that they have left to be able to respond in a mindful way and resist persuasion. Concerns for self-regulation at that point will outweigh economic concerns, with the consequence that their self-control will suffer later on. The research in Chapter 4 has shown that a forewarning of an influence attempt can make future self-regulation concerns (being able to resist that upcoming influence attempt) predominate people's concerns for current self-regulation. People will temporarily economize on their resources, and let them suffer on an intermediate self-control task, to have them ready when it comes to subsequent persuasion. As far as the role of selfregulation is concerned, the amount of regulatory resources one has left and one's future demands for self-control seem to jointly determine the style of information processing that consumers will adopt and the decisions they will make in an influence situation.

In addition to the popular two-system models of cognitive processing (the elaboration likelihood model, Petty \& Cacioppo, 1986; Petty \& Wegener, 1999; and the heuristicsystematic model of persuasion, Chaiken, 1980), the reflective-impulsive model by Strack and Deutsch (2004) explains (consumer) behavior as the joint outcome of reflective and impulsive mechanisms. According to this model, if consumers are motivated to engage in thorough information processing (e.g., when the consequences of a decision are important) and the situation allows them to invest the necessary time and cognitive capacity, consumer decision making will most likely be determined by reflective operations. However, when processing resources are insufficiently available, impulsive processes will get the upper hand (Strack, Werth, \& Deutsch, 2006). Importantly, according to Vohs (2006), the reflective system is driven by self-regulatory resources, being the underlying energy resource. Self-regulatory resources enable the reflective system to influence behavior, but when self-regulatory resource levels are low, this will render the reflective system unable to operate and consumer behavior will be more a 
result of impulse. Hofmann, Rauch, and Gawronski (2007) tested this prediction, and indeed found that resource depletion moderated the impact of reflective and impulsive precursors on consumer behavior. The amount of candy participants ate during a taste-and-rate task was primarily predicted by automatic affective reactions (impulsive precursors) in participants who were depleted by an emotion suppression task, but not in control participants. In contrast, candy consumption was primarily predicted by dietary restraint standards (reflective precursors) in participants who were not depleted of self-regulatory resources. The present research fits well with this theoretical model of consumer behavior, in that it endorses that self-regulatory mechanisms and types of cognitive processing are strongly intertwined. When people's resources for selfregulation are lowered during the initial request stage of an influence technique, this will render the reflective system unable to operate and consumer behavior will be more a result of impulse.

By linking up a 'classic' persuasion theme with contemporary insights on self-regulatory functioning, the present research also contributes to our knowledge in the domain of research on forewarnings of persuasion. It shows that the effects of forewarning of persuasive intent are a joint function of people's motivation and ability to withstand an unwanted upcoming influence attempt. As demonstrated in previous experiments (e.g., Petty \& Cacioppo, 1977), forewarning of an impending influence attempt encourages more systematic processing and stimulates the generation of counterarguments. The present research has now provided insight in the self-regulatory dynamics underlying this effect. A forewarning appears to be especially beneficial to those low in self-control strength, since it functions as a strong motivational factor for these individuals to build up their resistance.

As discussed above, the present research has important implications and contributions for theories and research on social influence processes. In addition, the present research offers new perspectives on research in the domain of self-regulation. First, and in a 
general sense, the present studies add to our understanding of the inner workings of self-control. As recently discussed by Baumeister and Vohs (2007), a state of selfregulatory resource depletion renders the self temporarily less able, but also less willing to function normally or optimally. Depletion is not solely a loss of resources since a reduction in self-control resources can be temporarily overcome and compensated by motivation. On the other hand, depletion is not solely a loss of motivation either, since recent studies by Gailliot, Baumeister et al. (2007) have shown that regulatory resources are rooted in physical energy stores. The present research underscores the notion that the ability and motivation to self-regulate are strongly related, and pertain to the idea that self-regulation involves a constant (likely unconscious) trade-off between multiple self-control demands.

Second, the present findings point to a previously unexplored 'theatre of operations' of principles involved in effortful self-regulation: that of dyadic social influence. Although the realm of interpersonal functioning has recently been addressed by self-regulatory resource depletion research (e.g., Vohs et al. 2005; Vohs \& Finkel 2006), instrumental dyadic interactions in which one party (the agent) tries to tempt or persuade the other party (the target) into behaving in a specific manner (e.g., donating to charity, acting as a volunteer) have been neglected as a manifestation of self-regulation. This omission is striking when considering that effortful self-presentation (cf. Vohs et al. 2005), a process known to rely on self-regulatory resources, has been stressed as an important topic in dyadic influence settings. However, a close examination of this research reveals a focus on regulation processes related to the influence agent, rather than the target (e.g., Forgas 2007; McFarland, Challagalla, \& Shervani, 2006; Payan \& McFarland, 2005). Hence, the current work forges new ground by illuminating the role of self-regulation on the target's part of a potential influence interaction. 
Third, it is crucial to highlight the socially desirable nature of the effects reported in our studies, and the fact that our results show that self-regulatory resource depletion can result in prosocial behavior. Our participants with low (temporary or chronic) selfcontrol were prepared to act as a volunteer, participate in future studies, do unpaid work for their university, and donate real money to charity. Whereas previous work has suggested that resource depletion results in an enhanced tendency to serve the self (Finkel \& Campbell, 2001; Tangney et al., 2004), the present findings attest to the notion that depletion can also result in an enhanced tendency to serve others.

\section{Potential limitations and directions for future research}

A potential limitation of the findings reported in Chapters 2 and 3 of this dissertation pertains to the fact that in the experiments where a full sequential request technique was used, the target request followed the initial request after a few minutes (see Experiment 2.4, and additionally Experiments 1 and 6 from Fennis et al., 2009; in the first three experiments of Chapter 2 the initial request was merely a conceptual variable, since no actual target request followed). This leaves open the question of what would occur with a larger delay between initial and target request. We could expect the time delay to act as a buffer against the 'hangover-effect' produced by the depleting initial request. However, this need not necessarily result in reduced compliance with the target request, as studies by Freedman and Fraser (1966) and others (e.g., Pliner et al., 1974) have shown. In all likelihood, however, compliance in these conditions would be the product of mindfulness governed by more controlled self-regulation processes, rather than depletion-induced mindlessness. The role of self-regulation in mindful compliance constitutes a promising venue for future research. In line with the notions tested in the present work, we would hypothesize that mindful compliance (or resourceful compliance) becomes likely when the influence script includes strong, compelling issue-relevant information, rather than the decisional heuristics featured in the present experiments. 
As a sideline, and also discussed in Chapter 2, one might wonder to what extent our studies reflect the 'classic' manipulations of compliance-gaining as used by Freedman and Fraser (1966) and others (e.g., Pliner et al. 1974) in which participants were asked for agreement with a small request before the larger target request was posed. At first glance, this procedure appears at odds with the present work which focused on the extent of such initial agreement (i.e., the extent to which initial agreement involved effortful responding). However, a key driver in the research by Freedman and Fraser (1966) also proved to be the extent of performance of the initial request, rather than agreement per se. Furthermore, meta-analytic findings also align with our main argument that it is not the act of initial agreement per se that is the decisive factor in producing compliance, but rather the extent of performance of the initial request (see Burger, 1999).

Another point of discussion is that at first sight the influence contexts and persuasive requests that were used in the present research might not seem prototypical for the requests that consumers have to deal with in everyday commercial situations (e.g., requests to actually purchase a product item). However, most of them are similar to the requests consumers often receive, like donating money to charity and filling out questionnaires for research organizations. As in most common real-life consumer situations, participants in the present studies were presented with offers they were not planning to yield to in advance, and they were persuaded to engage in activities without necessarily expecting a return on their investment (e.g., voluntarily participate in future studies of an unknown research society). Moreover, all influence techniques and requests that were targeted at the participants in the present studies incorporated either one of the social norms or heuristic principles that are typically being addressed by influence professionals when trying to persuade consumers into compliance: the principles of reciprocity, authority and likeability featured in our studies. Nevertheless, future research could more directly address the more 'typical' consumer situations, like personal sales and mediated commercial messages that are regulatory resource consuming. To the extent that these prototypical consumer settings also employ the principles of influence outlined by Cialdini and others 
(e.g., Cialdini, 1993), which they typically do, we expect the same mechanisms operating in these contexts.

In addition, future research may explore boundary conditions to the present two-stage model. For example, research may assess the conditions under which self-regulation failure may hinder, rather than foster, compliance. One possibility may lie in the type of heuristic present in the influence context. In the current work, self-regulatory resource depletion did not result in compliant behavior per se but increased reliance on salient heuristics; depleted participants yielded to the request of the influence agent because the reciprocity, authority, or liking principle informed them to do so. Hence, it suggests that compliance in these social situations was the 'easy way out' for resource-depleted individuals. Rather than maintaining a high level of resource-consuming resistance to the influence attempt, succumbing to the influence tactic and engaging in various acts of compliance presented itself as an efficient behavioral option in the decision-making context insofar as it brought immediate relief from a potentially taxing interpersonal encounter. Whereas in the current work the heuristics uniformly pointed to compliance, this need not necessarily be so. For instance, certain decisional heuristics, such as simple warnings of persuasive attempts (e.g., 'never trust a salesman with a slick suit') may well move the consumer away from compliance and, therefore, may foster resistance to the influence attempt. An additional venue might constitute addressing conditions under which responding to an initial request would not result in self-regulatory resource depletion but instead may'replenish'resources. One possibility might lie in an initial request that involves self-affirmation rather than active impression management (Steele, 1988), a variable that curtails the adverse effects of repeated acts of active self-regulation (Schmeichel \& Vohs, 2008). These strands of research would aid in delineating when sequential request influence techniques drain or replenish the self and hence when they would increase or decrease compliance. Notwithstanding qualifying conditions, the bulk of compliance-gaining procedures as studied by academics as well as performed by fundraisers, social- and commercial marketers involve techniques for which the proposed two-stage model will likely hold. Techniques such as the foot-in- 
the-door, that's-not-all, low-ball, fear-then-relief, multiple de-escalation, bait-and-switch, disrupt-then-reframe and door-in-the face, fall under the broad rubric of tactics to which the two-stage model applies.

Among these, the disrupt-then-reframe (DTR) and the door-in-the-face (DITF) techniques warrant special attention. In Chapter 2 it was discussed that research has shown that the disruption (the odd element) in the DTR results in reduced counterargumentation, which is a consequence of reduced self-control (Wheeler et al., 2007). In light of the limited-resource model, this finding is of interest because it suggests the existence of additional sources of depletion in social influence settings. Note that self-regulatory resource depletion emerges as the product of an effortful process, when individuals actively override their initial responses, emotions, or thoughts. In case of the DTR it is not an intrapsychic but an extrapsychic source that is responsible for the effort required in overriding the initial response and may produce the state of regulatory resource depletion: the disruption in the DTR. Recent research (Kardes et al., 2007) suggests that this disruption may be particularly burdensome for individuals high (as opposed to low) in the need for cognitive closure. Future research might profitably explore whether these individuals indeed experience higher levels of self-regulatory resource depletion than their low need for closure counterparts after responding to the disruption in the DTR.

Another venue of research might address the effectiveness of the door-in-the-face (DITF) technique from the limited-resource perspective. At first glance, one might argue that a DITF technique should deplete resources more so than should a foot-in-the-door (FITD) script because the initial request is typically larger in a DITF. However, work by Tybout, Sternthal, and Calder (1983) suggests that the DITF is more effective when the magnitude of the initial request decreases rather than increases. Nevertheless, these results may well fit the limited-resource paradigm because note that the impact of the DITF hinges on the target's refusal of the initial request. This refusal may entail active self-regulation. That is, being counternormative in terms of politeness in interpersonal 
interactions, refusal responses typically induce social costs and hence may trigger active self-presentation concerns (e.g., avoiding embarrassment, Shoemaker, Eichholz, \& Skewes, 2002). Indeed, recent research (Flynn \& Lake, 2008) suggests that refusing a request often costs more than accommodating one, a fact that is often (and ironically) lost to the agent. We can easily imagine that having to refuse a more moderate, reasonable initial request is harder and requires more self-regulatory resources than refusing an absurdly large initial request, and that the former therefore prompts more effortful impression management concerns. As the present results suggest, this type of refusal may promote a state of self-regulatory resource depletion, which would explain the relative effectiveness of the more moderate DITF, a hypothesis that awaits future testing. Further research could also investigate whether other compliance-gaining techniques, that do not involve a series of initial requests (such as the fear-then-relief procedure, Dolinski \& Nawrat, 1998), are effective through self-regulatory resource depletion or other routes.

A last note on the type of requests that were used in the present research refers to the fact that people in our studies were presented with persuasive requests that were relatively unsolicited and expected to be received with mild resistance. Future research might address what would happen when a request elicits an initial positive response. Will a state of self-regulatory resource depletion in that case still increase the chances of compliance? We expect that in these instances depletion will still evince an effect on compliance through the use of a compliance-promoting heuristic.

A point that also receives attention is that low self-regulatory ability that has been dispositionally instead of situationally determined has also been shown to entail a larger susceptibility to influence. Future research might explore what other individual difference variables related to self-control ability could affect persuasion processes. Preliminary data suggest that people high in self-monitoring (the extent to which people observe and control their self-presentation and expressive behavior, Snyder, 
1974) become more depleted of their self-control resources than low self-monitors after yielding to an initial request with high self-presentational demands. High self-monitors have higher impression management concerns and are therefore probably more willing to invest considerable effort in responding to this type of initial request. Future research could further explore whether a full social influence strategy that addresses self-presentational concerns is more successful when applied to high self-monitors as opposed to low self-monitors.

Finally, another important venue for future research would be exploring the link between self-regulatory resource depletion and other cognitive psychological processes, like distraction or cognitive load. In contrast to the temporary unavailability of cognitive resources for the task at hand when one is distracted or under cognitive load, selfregulatory resource depletion distinguishes itself by the characteristic 'hangover-effect' (Vohs, 2006), in that one act of self-regulation leads to poorer self-control subsequently. Nevertheless, one could argue that the observed effects of reduced self-control performance in the present studies are a result of cognitive load resulting from fatigue and exhaustion induced by the resource depletion manipulations. However, and as recently addressed by Fischer, Greitemeyer, and Frey (2008), we strongly believe that resource depletion and cognitive load are distinct psychological concepts. Whereas cognitive load is purely cognitive in nature (as it taxes working memory), resource depletion also incorporates a motivational aspect, as has been shown by the studies in Chapter 4. Whereas cognitive load simply taxes our cognitive resources, which makes people temporarily less able to engage in regulated, systematic processing, the ability to perform self-control over our cognitions and behaviors is not only determined by the amount of cognitive resources one has available, but is strongly intertwined with the motivational aspect of willpower to use these resources or avoid using any more of them. Future research should try to carefully distinguish between these cognitive processes and determine in which respect they are or are not related to each other. 
A final note referring to important venues for future research to explore, is whether the effects that were found in the present studies also hold when persuasion effects are measured on an implicit level (e.g., consumer preferences measured with an Implicit Association Test; Brunel, Tietje, \& Greenwald, 2004) instead of explicitly. One could expect that for individuals low in self-control, implicit and explicit measures of persuasion converge to a higher extent than for those high in self-control. Whereas depleted and non-depleted individuals will respond in a similar fashion to persuasion attempts on an implicit level, they will respond differently when explicitly confronted with persuasion: consumers low in self-control likely do not have the resources available, or are not willing to use their resources to override the impulsive, acquiescent behaviors that are being elicited from them by influence agents, whereas consumers high in self-control do have their resources available to actively override these impulses.

\section{Practical implications}

The findings reported in the present dissertation not only contribute to the scientific fields of self-regulation and social influence, but will appeal to people outside academia as well. It is not difficult to imagine how the insights provided by the present research can be applied in practice. Thereby one could adopt the perspective of an influence professional that might profit from the knowledge generated by the present studies, but one could also adopt the perspective of the consumer, and think about the way the present findings are insightful for consumers and consumer-related organisations.

From an influence agent's point of view, the present findings provide influence practitioners with a unique insight into consumers' minds. Knowing when consumers' controlled and purposeful self fails in making decisions gives the tools right in hand to refine persuasion tactics and strike under conditions when consumers' self-regulatory resource are suboptimal. Sales representatives and fundraisers are probably more successful if they make use of initial requests to such an extent that consumers become 
deprived of their regulatory resources. In this state of mind the consumer will be likely to "follow the path of least resistance" and will be more likely to engage in normative or routine behavior. However, we assume that most compliance professionals already use these tools, yet without being aware of the specific psychological processes that determine the effectiveness of their tactics. Though influence professionals will call it experience and intuition, they already tend to catch people when they're off guard, engaged in other (self-regulatory) activities (e.g., shopping and meanwhile conversing with friends), or depleted by previous self-regulatory tasks (e.g., organising the family household after a day of hard work). Therefore, on a practical note we expect the present research merely to confirm that what influence professionals already do, is effective.

When adopting a consumer perspective, the current work is likely to contribute to consumers' 'persuasion knowledge' (Friestad \& Wright, 1994), which incorporates theories and beliefs about how persuasion agents try to influence us and how we can cognitively and physically deal with these situations of influence. This persuasion knowledge will tell us to be on guard when entering a marketing environment, and could (unconsciously) function as a forewarning when presented with an unsolicited request. It will help consumers to keep their wits about them, not let themselves become mindless 'victims' of other people's wishes, and see through the ploys that become increasingly transparent to them, even when they are used in the interest of the noblest of causes.

\section{Concluding remarks}

The research presented in this dissertation has shown that a central aspect of human functioning, the ability and motivation to regulate the self, is a main determinant of our success in dealing with (unwanted) persuasion. It is natural to feel a strong pull to behave in accordance with someone else's wishes when that person is an intimate other but very little reason to do so otherwise, especially when there is no direct or 
immediate 'return on investment' as is the case with commercial requests. Fundraisers, marketers, and other influence agents have perfected the art of gaining compliance from consumers they have never met before and may well never encounter again. How they do it has been a decades-long mystery at which behavioral scientists have been cracking away. Our research reveals that one key feature of effective influence tactics is the wearing down of self-regulatory resources that would otherwise be put toward resistance. However, we should also be comforted by the present research that under the right circumstances, consumers are still proficient in defending themselves. Although far from solving the puzzle, it surely is advantageous in this era of influence attempts to have even one more piece put into place. 




\section{References}



Aiken, L. S., \& West, S. G. (1991). Multiple Regression: Testing and Interpreting Interactions. Newbury Park, CA: Sage.

Allyn, J., \& Festinger, L. (1961). The effectiveness of unanticipated persuasive communications. Journal of Abnormal and Social Psychology, 62(1), 35-40.

Asch, S. E. (1951). Effects of group pressure upon the modification and distortion of judgment. In H. Guetzkow (Ed.), Groups, leadership, and men (pp. 177-190). Oxford, England: Carnegie Press.

Asch, S. E. (1956). Studies of independence and conformity: A minority of one against unanimous majority. Psychological Monographs, 70(9, Whole No. 416).

Bargh, J. A. (1997). The automaticity of everyday life. In R. S. Jr. Wyer (Ed.), The automaticity of everyday life: Advances in social cognition (Vol. 10, pp. 1-61). Mahwah, NJ: Lawrence Erlbaum.

Baron, R. M., \& Kenny, D. A. (1986). The moderator-mediator variable distinction in social psychological research: Conceptual, strategic and statistical considerations. Journal of Personality and Social Psychology, 51(6), 1173-1182.

Baumeister, R. F. (2002). Yielding to temptation: Self-control failure, impulsive purchasing, and consumer behavior. Journal of Consumer Research, 28(4), 670-676.

Baumeister, R. F., Bratslavsky, E., Muraven, M., \& Tice, D. M. (1998). Ego depletion: Is the active self a limited resource? Journal of Personality and Social Psychology, 74(5), 1252-1265.

Baumeister, R. F., Muraven, M., \& Tice, D. M. (2000). Ego depletion: A resource model of volition, self-regulation, and controlled processing. Social Cognition, 18(2), 130-150.

Baumeister, R. F., Schmeichel, B. J., \& Vohs, K. D. (2007). Self-regulation and the executive function: The self as controlling agent. In A. W. Kruglanski \& E. T. Higgins (Eds.), Social psychology: Handbook of basic principles (2nd ed., pp. 516539). New York: Guilford Press.

Baumeister, R. F., Sparks, E. A., Stillman, T. F., \& Vohs, K. D. (2008). Free will in consumer behavior: Self-control, ego depletion, and choice. Journal of Consumer Psychology, 18(1), 4-13. 
Baumeister, R. F., \& Vohs, K. D. (2004). Handbook of self-regulation: Research, theory, and applications. New York: Guilford Press.

Baumeister, R. F., \&Vohs, K. D. (Eds.). (2007). Self-regulation, ego depletion and motivation. Social and Personality Psychology Compass, 1(1), 115-128.

Baumeister, R. F., Vohs, K. D., \& Funder, D. C. (2007). Psychology as the science of selfreports and finger movements: Whatever happened to actual behavior? Perspectives on Psychological Science, 2(4), 396-403.

Baumeister, R. F., Vohs, K. D., \& Tice, D. M. (2007). The strength model of self-control. Current Directions in Psychological Science, 16(6), 351-355.

Bobko, P. (1986). A solution to some dilemmas when testing hypotheses about ordinal interactions. Journal of Applied Psychology, 71(2), 323-326.

Briñol, P., \& Petty, R. E. (2005). Individual differences in persuasion. In D. Albarracin, B. T. Johnson, \& M. P. Zanna (Eds.), The handbook of attitudes and attitude change (pp. 575-615). Hillsdale, NJ: Erlbaum.

Brock, T. C. (1967). Communication discrepancy and intent to persuade as determinants of counterargument production. Journal of Experimental Social Psychology, 3(3), 269-309.

Brunel, F. F., Tietje, B. C., \& Greenwald, A. G. (2004). Is the implicit association test a valid and valuable measure of implicit consumer social cognition? Journal of Consumer Psychology, 14(4), 385-404.

Bruyneel, S., Dewitte, S., Vohs, K. D., \& Warlop, L. (2006). Repeated choosing increases susceptibility to affective product features. International Journal of Research in Marketing, 23(2), 215-225.

Burger, J. M. (1986). Increasing compliance by improving the deal: The that's-not-all technique. Journal of Personality and Social Psychology, 51(2), 277-283.

Burger, J. M. (1999). The foot-in-the-door compliance procedure: A multiple process analysis and review. Personality and Social Psychology Review, 3(4), 303-325. 
Burger, J. M., \& Cornelius, T. (2003). Raising the price of agreement: Public commitment and the lowball compliance procedure. Journal of Basic and Applied Social Psychology, 33(5), 923-934.

Burger, J. M., \& Petty, R. E. (1981). The low-ball compliance technique: Task or person commitment? Journal of Personality and Social Psychology, 40(3), 492-500.

Burkley, E. (2008). The role of self-control in resistance to persuasion. Personality and Social Psychology Bulletin, 34(3), 419-431.

Chaiken, S. (1980). Heuristic versus systematic information processing and the use of source versus message cues in persuasion. Journal of Personality and Social Psychology, 39(5), 752-766.

Chaiken, S., \& Trope, Y. (1999). Dual process theories in social psychology. New York: Guilford Press.

Chen, H. C., Reardon, R., Rea, C., \& Moore, D. J. (1992). Forewarning of content and involvement: Consequences for persuasion and resistance to persuasion. Journal of Experimental Social Psychology, 28(6), 523-541.

Cialdini, R. B. (1993). Influence: Science and practice (3rd ed.). New York: Harper Collins.

Cialdini, R. B., Cacioppo, J. T., Bassett, R., \& Miller, J. A. (1978). Low-ball procedure for producing compliance: Commitment then cost. Journal of Personality and Social Psychology, 36(5), 463-476.

Cialdini, R. B., \& Goldstein, N. J. (2004). Social influence: Compliance and conformity. Annual Review of Psychology, 55, 591-621.

Cialdini, R. B., Vincent, J. E., Lewis, S. K., Catalan, J., Wheeler, D., \& Darby, B. L. (1975). Reciprocal concessions procedure for inducing compliance: The door-in the-face technique. Journal of Personality and Social Psychology, 31(2), 206215.

Ciarocco, N. J., Twenge, J. M., Muraven, M., \& Tice, D. M. (2010). The state self-control capacity scale: Reliability, validity, and correlations with physical and psychological stress. Monmouth University. Manuscript under revision. 
Davis, B. P., \& Knowles, E. S. (1999). A disrupt-then-reframe technique of social influence. Journal of Personality and Social Psychology, 76(2), 192-199.

Deutsch, M., \& Gerard, H. B. (1955). A study of normative and informational social influences upon individual judgment. Journal of Abnormal and Social Psychology, 51(3), 629-636.

Dewitte, S., Bruyneel, S., \& Geyskens, K. (2009). Self-regulating enhances self-regulation in subsequent consumer decisions involving similar response conflicts. Journal of Consumer Research, 36(3), 394-405.

Dolinski, D., \& Nawrat, R. (1998). 'Fear-then-relief' procedure for producing compliance: Beware when the danger is over. Journal of Experimental Social Psychology, 34(1), 27-50.

Faber, R. J., \& Vohs, K. D. (2004). To buy or not to buy?: Self-control and self-regulatory failure in purchase behavior. In R. F. Baumeister \& K. D. Vohs (Eds.), Handbook of self-regulation: Research, theory, and applications (pp. 509524). New York: Guilford Press.

Fennis, B. M., Das, E. H. H. J., \& Pruyn, A. Th. H. (2004). If you can't dazzle them with brilliance, baffle them with nonsense: Extending the impact of the disruptthen-reframe technique of social influence. Journal of ConsumerPsychology, 14(3), 280-290.

Fennis, B. M., Das, E. H. H. J., \& Pruyn, A. Th. H. (2006). Interpersonal communication and compliance: The disrupt-then-reframe technique in dyadic influence settings. Communication Research, 33(2), 136-151.

Fennis, B. M., \& Janssen, L. (under review). Mindlessness and social influence: Sequential request techniques foster compliance by draining self-control resources.

Fennis, B. M., Janssen, L., \& Vohs, K. D. (2009). Acts of benevolence: A limited-resource account of compliance with charitable requests. Journal of Consumer Research, 35(6), 906-924.

Fern, E. F., Monroe, K. B., \& Avila, R. A. (1986). Effectiveness of multiple request strategies: A synthesis of research results. Journal of Marketing Research, 23(2), 144152. 
Ferreira, M. B., Garcia-Marques, L., Sherman, S. J., \& Sherman, J. W. (2006). Automatic and controlled components of judgment and decision making. Journal of Personality and Social Psychology, 91(5), 797-813.

Finkel, E., \& Campbell, W. K. (2001). Self-control and accommodation in close relationships: An interdependence analysis. Journal of Personality and Social Psychology, 81(2), 263-277.

Fischer, P, Greitemeyer, T., \& Frey, D. (2008). Self-regulation and selective exposure: The impact of depleted self-regulation resources on confirmatory information processing. Journal of Personality and Social Psychology, 94(3), 382-395.

Fish, B., \& Kaplan, K. J. (1974). Does a 'foot-in-the-door' get you in or out? Psychological Reports, 34(1), 35-42.

Flynn, F. J., \& Lake, V. (2008). If you need help, just ask: Underestimating compliance with direct requests for help. Journal of Personality and Social Psychology, 95(1), 128-143.

Forgas, J. P. (2007). When sad is better than happy: Negative affect can improve the quality and effectiveness of persuasive messages and social influence strategies. Journal of Experimental Social Psychology, 43(3), 513-528.

Freedman, J. L., \& Fraser, S. C. (1966). Compliance without pressure: The foot-in-the-door technique. Journal of Personality and Social Psychology, 4(2), 195-202.

Freedman, J. L., \& Sears, D. O. (1965). Warning, distraction, and resistance to influence. Journal of Personality and Social Psychology, 1(3), 262-266.

Friestad, M., \& Wright, P. (1994). The persuasion knowledge model: How people cope with persuasion attempts. Journal of Consumer Research, 21(1), 1-31.

Gailliot, M. T., Baumeister, R. F., \& DeWall, C. N. (2007). Self-control relies on glucose as a limited energy resource: Willpower is more than a metaphor. Journal of Personality and Social Psychology, 92(2), 325-336.

Gailliot, M. T., Plant, A. E., Butz, D. A., \& Baumeister, R. F. (2007). Increasing selfregulatory strength can reduce the depleting effect of suppressing stereotypes. Personality and Social Psychology Bulletin, 33(2), 281-294. 
Gouldner, A. W. (1960). The norm of reciprocity: A preliminary statement. American Sociological Review, 25(1), 161-178.

Hass, R. G., \& Grady, K. (1975). Temporal delay, type of forewarning, and resistance to influence. Journal of Experimental Social Psychology, 11(5), 459-469.

Hofmann, W., Rauch, W., \& Gawronski, B. (2007). And deplete us not into temptation: Automatic attitudes, dietary restraint, and self-regulatory resources as determinants of eating behavior. Journal of Experimental Social Psychology, 43(3), 497-504.

Janssen, L., Fennis, B. M., \& Pruyn, A. Th. H. (2008). Zelfregulatieve bronuitputting en het gebruik van heuristische principes bij persuasieve beïnvloeding. In B. Beersma, R. Custers, F. van Harreveld, W. van Rijswijk, \& J. Karremans (Red.), Jaarboek Sociale Psychologie 2007 (pp. 159-172). Groningen: ASPO pers.

Janssen, L., Fennis, B. M., \& Pruyn, A. Th. H. (2010). Forewarned is forearmed: Conserving self-control strength to resist social influence. Manuscript under revision.

Janssen, L., Fennis, B. M., Pruyn, A. Th. H., \& Vohs, K. D. (2008). The path of least resistance: Regulatory resource depletion and the effectiveness of social influence techniques. Journal of Business Research, 61(10), 1041-1045.

Kardes, F. R., Fennis, B. M., Hirt, E. R., Tormala, Z. L., \& Bullington, B. (2007). The role of the need for cognitive closure in the effectiveness of the disrupt-thenreframe influence technique. Journal of Consumer Research, 34(3), 377-385.

Kiesler, C. A., \& Kiesler, S. B. (1964). Role of forewarning in persuasive communications. Journal of Abnormal and Social Psychology, 68(5), 547-549.

Langer, E. J. (1992). Matters of mind: Mindfulness/mindlessness in perspective. Consciousness and Cognition, 1(4), 289-305.

Langer, E. J., Blank, A., \& Chanowitz, B. (1978). The mindlessness of ostensibly thoughtful action: The role of 'placebic' information in interpersonal interaction. Journal of Personality and Social Psychology, 36(6), 635-642. 
Martijn, C., Alberts, H. J. E. M., Merckelbach, H., Havermans, R., Huijts, A., \& De Vries, N. K. (2007). Overcoming ego-depletion: The influence of exemplar priming on self-control performance. European Journal of Social Psychology, 37(2), 231-238.

McFarland, R. G., Challagalla, G. N., \& Shervani, T. A. (2006). Influence tactics for effective adaptive selling. Journal of Marketing, 70(4), 103-117.

Milgram, S. (1963). Behavioral study of obedience. Journal of Abnormal and Social Psychology, 67(4), 371-378.

Milgram, S. (1974). Obedience to authority. New York: Harper.

Muraven, M., Shmueli, D., \& Burkley, E. (2006). Conserving self-control strength. Journal of Personality and Social Psychology, 91(3), 524-537.

Muraven, M., \& Slessareva, E. (2003). Mechanism of self-control failure: Motivation and limited resources. Personality and Social Psychology Bulletin, 29(7), 894-906.

Muraven, M., Tice, D. M., \& Baumeister, R. F. (1998). Self-control as a limited resource: Regulatory depletion patterns. Journal of Personality and Social Psychology, 74(3), 774-789.

O'Keefe, D. J., \& Hale, S. L. (1998). The door-in-the-face influence strategy: A randomeffects meta-analytic review. Communication Yearbook, 21, 1-33.

O'Keefe, D. J., \& Hale, S. L. (2001). An odds-ratio-based meta-analysis of research on the door-in-the-face influence strategy. Communication Reports, 14(1), 31-38.

Orphans and Vulnerable Children (OVC) Sponsorship Program. Retrieved April 2, 2009, from http://www.navtifoundation.org/orphanvol-children.htm

Payan, J. M., \& McFarland, R. G. (2005). Decomposing influence strategies: Argument structure and dependence as determinants of the effectiveness of influence strategies in gaining channel member compliance. Journal of Marketing, 69(3), 66-79.

Petty, R. E., \& Cacioppo, J. T. (1977). Forewarning, cognitive responding, and resistance to persuasion. Journal of Personality and Social Psychology, 35(9), 645-655. 
Petty, R. E., \& Cacioppo, J. T. (1981). Attitudes and persuasion: Classic and contemporary approaches. Dubuque, IA: Wm. C. Brown.

Petty, R. E., \& Cacioppo, J. T. (1986). The elaboration likelihood model of persuasion. In L. Berkowitz (Ed.), Advances in experimental social psychology (Vol. 19, pp. 123-205). New York: Academic Press.

Petty, R. E., \& Wegener, D. T. (1999). The elaboration likelihood model: Current status and controversies. In S. Chaiken \& Y. Trope (Eds.), Dual-process theories in social psychology (pp.37-72). New York: Guilford Press.

Pliner, P., Hart, H., Kohl, J., \& Saari, D. (1974). Compliance without pressure: Some further data on the foot-in-the-door technique. Journal of Experimental Social Psychology, 10(1), 17-22.

Pocheptsova, A., Amir, O., Dhar, R., \& Baumeister, R. F. (2009). Deciding without resources: Resource depletion and choice in context. Journal of Marketing Research, 46(3), 344-355.

Pollock, C. L., Smith, S. D., Knowles, E. S., \& Bruce, H. J. (1998). Mindfulness limits compliance with the that's-not-all technique. Personality and Social Psychology Bulletin, 24(11), 1153-1157.

Quinn, E. P., Brandon, T. H., \& Copeland, A. L. (1996). Is task persistence related to smoking and substance abuse? The application of learned industriousness theory to addictive behaviours. Experimental and Clinical Psychopharmacology, 4(2), 186-190.

Schmeichel, B. J., \& Vohs, K. D. (2009). Self-affirmation and self-control: Affirming core values counteracts ego depletion. Journal of Personality and Social Psychology, 96(4), 770-782.

Schmeichel, B. J., Vohs, K. D., \& Baumeister, R. F. (2003). Intellectual performance and ego depletion: Role of the self in logical reasoning and other information processing. Journal of Personality and Social Psychology, 85(1), 33-46.

Seligman, C., Bush, M., \& Kirsch, K. (1976). Relationship between compliance in the foot-in-the-door paradigm and size of first request. Journal of Personality and Social Psychology, 33(5), 517-520. 
Sherif, M. (1936). The psychology of social norms. Oxford, England: Harper.

Shoemaker, P. J., Eichholz, M., \& Skewes, E. A. (2002). Item nonresponse: Distinguishing between don't know and refuse. International Journal of Public Opinion Research, 14(2), 193-201.

Smit, A. S., Eling, P. A. T. M., \& Coenen, A. M. L. (2004). Mental effort causes vigilance decrease due to resource depletion. Acta Psychologica, 115(1), 35-42.

Snyder, M. (1974). Self-monitoring of expressive behavior. Journal of Personality and Social Psychology, 30(4), 526-537.

Steele, C. M. (1988). The psychology of self-affirmation: Sustaining the integrity of the self. In L. Berkowitz (Ed.), Advances in Experimental Social Psychology (Vol. 21, pp. 261-302). New York: Academic Press.

Strack, F., \& Deutsch, R. (2004). Reflective and impulsive determinants of social behavior. Personality and Social Psychology Review, 8(3), 220-247.

Strack, F., Werth, L., \& Deutsch, R. (2006). Reflective and impulsive determinants of consumer behavior. Journal of Consumer Psychology, 16(3), 205-216.

Stroop, J. R. (1935). Studies of interference in serial verbal reactions. Journal of Experimental Psychology, 18(6), 643-662.

Tangney, J. P., Baumeister, R. F., \& Boone, A. L. (2004). High self-control predicts good adjustment, less pathology, better grades and interpersonal success. Journal of Personality, 72(2), 271-322.

Tice, D. M., Baumeister, R. F., Shmueli, D., \& Muraven, M. (2007). Restoring the self: Positive affect helps improve self-regulation following ego-depletion. Journal of Experimental Social Psychology, 43(3), 379-384.

Tybout, A. M. (1978). Relative effectiveness of three behavioral influence strategies as supplements to persuasion in a marketing context. Journal of Marketing Research, 15(2), 229-242.

Tybout, A. M., Sternthal, B., \& Calder, B. J. (1983). Information availability as a determinant of multiple request effectiveness. Journal of Marketing Research, 20(3), 280290. 
Vohs, K. D. (2006). Self-regulatory resources power the reflective system: Evidence from five domains. Journal of Consumer Psychology, 16(3), 217-223.

Vohs, K. D., Baumeister, R. F., \& Ciarocco, N. J. (2005). Self-regulation and selfpresentation: Regulatory resource depletion impairs impression management and effortful self-presentation depletes regulatory resources. Journal of Personality and Social Psychology, 88(4), 632-657.

Vohs, K. D. , Baumeister, R. F., Schmeichel, B. J., Twenge, J. M., Nelson, N. M., \& Tice, D. M. (2008). Making choices impairs subsequent self-control: A limited resource account of decision making, self-regulation, and active initiative. Journal of Personality and Social Psychology, 94(5), 883-898.

Vohs, K. D., \& Faber, R. J. (2007). Spent resources: Self-regulatory resource availability affects impulse buying. Journal of Consumer Research, 33(4), 537-547.

Vohs, K. D., \& Finkel, E. J. (2006). Self and Relationships: Connecting Intrapersonal and Interpersonal Processes. New York: Guilford Press.

Vohs, K. D., \& Heatherton, T. F. (2000). Self-regulatory failure: A resource-depletion approach. Psychological Science, 11(3), 249-254.

Vohs, K. D., Lasaleta, J., \& Fennis, B. M. (in press). Self-regulation in the interpersonal sphere. In J. Forgas, R. F. Baumeister, \& D. Tice (Eds.), Cognitive, affective, and motivational processes. Psychology Press.

Vohs, K. D., Mead, N. L., \& Goode, M. R. (2006). The psychological consequences of money. Science, 314(5802), 1154-1156.

Wallace, H. M., \& Baumeister, R. F. (2002). The effects of success versus failure feedback on further self-control. Self and Identity, 1(1), 35-41.

Watson, D. L., Clark, L. A., \& Tellegen, A. (1988). Development and validation of brief measures of positive and negative affect: The PANAS scales. Journal of Personality and Social Psychology, 54(6), 1063-1070.

Webb, T. L., \& Sheeran, P. (2003). Can implementation intentions help to overcome egodepletion? Journal of Experimental Social Psychology, 39(3), 279-286. 
Wheeler, S. C., Briñol, P., \& Hermann, A. D. (2007). Resistance to persuasion as selfregulation: Ego-depletion and its effects on attitude change processes. Journal of Experimental Social Psychology, 43(1), 150-156.

Wood, W., \& Quinn, J. M. (2003). Forewarned and forearmed? Two meta-analytic syntheses of forewarnings of influence appeals. Psychological Bulletin, 129, 119-138. 



\section{Appendix}





\section{State Ego Depletion Scale}

\section{(Ciarocco, Twenge, Muraven, \& Tice, 2010)}

Please respond to the statements below, describing how you feel right now. We are interested in your feelings at this moment. Use the following scale to record your answers:

$1=$ not true

$2=$ a little not true

$3=$ somewhat not true

$4=$ neutral

$5=$ a little true

$6=$ somewhat true

$7=$ very true

1. I feel mentally exhausted.

2. Right now, it would take a lot of effort for me to concentrate on something.

3. I need something pleasant to make me feel better.

4. I feel motivated. $\boldsymbol{R}$

5. If I were given a difficult task right now, I would give up easily.

6. I feel drained.

7. I have lots of energy. $\boldsymbol{R}$

8. I feel worn out.

9. If I were tempted by something right now, it would be very difficult to resist.

10. I would want to quit any difficult task I were given.

11. I feel calm and rational. $\boldsymbol{R}$

12. I can't absorb any more information.

13. I feel lazy.

14. Right now I would find it difficult to plan ahead. 
15. I feel sharp and focused. $\boldsymbol{R}$

16. I want to give up.

17. This would be a good time for me to make an important decision. $\boldsymbol{R}$

18. I feel like my willpower is gone.

19. My mind feels unfocused right now.

20. I feel ready to concentrate. $\boldsymbol{R}$

21. My mental energy is running low.

22. A new challenge would appeal to me right now. $\boldsymbol{R}$

23. I wish I could just relax for awhile.

24. I am having a hard time controlling my urges.

25. I feel discouraged.

$\boldsymbol{R}=$ reverse scored 




\section{Samenvatting}

(Summary in Dutch) 

aarom gaan consumenten in op ongevraagde verzoeken? Het is een intrigerend onderwerp dat onderzoekers binnen het onderzoeksveld van de marketingcommunicatie en consumentenpsychologie al jarenlang met veel passie bestuderen. We komen in ons dagelijks leven veelvuldig in aanraking met verkopers, fondsenwervers of andere beïnvloedingsprofessionals die ons proberen over te halen om tijd, geld of moeite te investeren in het steunen van hun doelen of organisaties. Hoewel we dit meestal niet van plan zijn, gaan we geregeld op dergelijke verzoeken in, zonder daar noodzakelijkerwijs zelf op vooruit te gaan. Zo spreek ik uit eigen ervaring als ik zeg dat je na een kort gesprek met een straatverkoper lid kunt worden van een boekenclub, ook als je boeken eigenlijk liever in een echte winkel koopt op momenten dat het jou zelf het beste uitkomt. Wat maakt het voor consumenten vaak zo moeilijk om in dergelijke situaties weerstand te bieden aan beïnvloeding en wanneer slagen zij hier juist wel in?

Het onderzoek in dit proefschrift heeft deze vragen benaderd vanuit een perspectief van zelfregulatie. Daarmee wordt gesuggereerd dat de wijze waarop mensen omgaan met (ongewenste) beïnvloeding voor een belangrijk deel afhankelijk is van de mogelijkheid om actief controle uit te oefenen over het eigen gedrag. Het tijdelijk falen van onze zelfregulatie ligt mogelijk ten grondslag aan vele vormen van beïnvloeding. Het huidige onderzoek heeft zich daarbij niet alleen gericht op het vermogen tot zelfcontrole, maar heeft ook getoetst hoe de motivatie om efficiënt met zelfregulatieve middelen om te gaan, bepalend is voor de manier waarop mensen reageren op persuasieve verzoeken. Dit proefschrift levert daarmee een bijdrage aan een beter begrip van de manier waarop wij omgaan met informatie en ons gedragen in sociale beïnvloedingssituaties.

Om de rol van zelfregulatie te kunnen bestuderen in een sociale beïnvloedingscontext heeft het huidige onderzoek zich geconcentreerd op sociale beïnvloedingstechnieken. Onderzoek heeft veelvuldig aangetoond dat mensen makkelijker te overreden zijn wanneer zij worden onderworpen aan een beïnvloedingsscript, dan wanneer hen een 
verzoek wordt gedaan zonder 'warming-up' (Burger, 1999; Cialdini \& Goldstein, 2004). Een grote verscheidenheid aan sociale beïnvloedingstechnieken is wetenschappelijk onderzocht, waaronder de foot-in-the-door techniek, waarbij het daadwerkelijke verzoek voorafgegaan wordt door een kleiner, moeilijk te weigeren verzoek (Freedman \& Fraser, 1966), en de door-in-the-face strategie, waarbij het weigeren van een relatief groot verzoek aan het daadwerkelijke verzoek voorafgaat (Cialdini et al., 1975).

Bij het verklaren van de effectiviteit van deze technieken speelt het principe van automaticiteit een belangrijke rol (Cialdini, 1993; Cialdini \& Goldstein, 2004). In tegenstelling tot het nauwkeurig en kritisch afwegen van de voor- en nadelen van een verzoek of aanbod, blijken mensen relatief gedachteloos te reageren (met weinig bewuste aandacht en inspanning) wanneer zij worden blootgesteld aan een sociale beïnvloedingstechniek. Mensen vallen terug op gewoonten en routine, en maken automatisch gebruik van mentale 'shortcuts' of heuristieken bij het maken van een beslissing. Het gebruik van heuristieken (zoals het 'voor-wat-hoort-wat' principe) zal over het algemeen de kans op instemmen met een verzoek vergroten (Cialdini, 1993).

De vraag die centraal staat in dit proefschrift is waarom mensen vaak automatisch en gedachteloos reageren in beïnvloedingssituaties. Hoe kunnen we verklaren dat mensen met relatief weinig cognitieve inspanning handelen wanneer zij worden blootgesteld aan een beïnvloedingstechniek en automatisch gebruik maken van heuristieken bij het nemen van een beslissing? Een mogelijke verklaring voor deze gedachteloosheid kan gevonden worden in een kenmerk dat vrijwel alle succesvolle beïnvloedingstechnieken met elkaar gemeen hebben: meervoudige beslissingsmomenten of opeenvolgende verzoeken (Fern et al., 1986). Men wordt onderworpen aan een initieel verzoek, waarbij men een serie vragen beantwoordt of meerdere keuzes maakt, voordat het doelverzoek wordt gepresenteerd. Aangezien men tijdens de initiële fase van een beïnvloedingstechniek ertoe wordt aangezet om doelbewust, bedachtzaam en actief te antwoorden, is het waarschijnlijk dat deze initiële fase zelfcontrole vereist en het vermogen tot zelfregulatie 
doet afnemen. Volgens het 'limited-resource model of self-control' (Baumeister, Vohs, \& Tice, 2007) put elke doelbewuste en gereguleerde handeling van het 'zelf' uit een mentale energiebron, die verwant is aan kracht en energie en daardoor eindig is. Na een handeling die zelfcontrole vereist, zijn mensen tijdelijk minder goed in staat tot actieve zelfregulatie (een staat die'self-regulatory resource depletion' wordt genoemd) en zal het doelbewuste zelf tijdelijk minder goed functioneren. Men wordt gevoeliger voor heuristische informatie en gaat sterker vertrouwen op impulsieve en automatische vormen van informatieverwerking en gedrag (Baumeister \& Vohs, 2007; Vohs et al., 2005), hetgeen kenmerkend is voor een toestand van gedachteloosheid. Het is daarom waarschijnlijk dat een tijdelijk verminderd vermogen tot zelfregulatie ontvankelijker maakt voor beïnvloeding: wanneer in de beïnvloedingscontext een geschikte heuristiek aanwezig is die instemming met een verzoek bevordert, zal een verminderd vermogen tot zelfregulatie de kans verhogen dat men instemt met het doelverzoek van een beïnvloedingstechniek. Heuristieken die de kans op instemmen verhogen zijn o.a. de principes van wederkerigheid, autoriteit en sympathie (zie Cialdini's principes van beïnvloeding, 1993).

De kern van dit proefschrift wordt gevormd door de hypothese dat zelfregulatie een onderliggende factor is die de effectiviteit van sociale beïnvloedingstechnieken zou kunnen verklaren. Deze hypothese is weergegeven in een 2-fasen model (zie Figuur 1, Hoofdstuk 1). De veronderstellingen van dit model zijn getoetst in een serie van acht experimenten, die ofwel in een laboratorium, ofwel in een natuurlijke setting zijn uitgevoerd. In Hoofdstukken 2 en 3 worden deze studies uitvoerig besproken. Hoofdstuk 4 vormt een aanvulling op de veronderstellingen van dit model en doet verslag van drie experimenten die zich richten op de motivationele aspecten van zelfregulatie. Wanneer het vermogen tot zelfregulatie tijdelijk is afgenomen, blijkt het efficiënt omgaan met resterende zelfregulatieve middelen een succesvolle (onbewuste) strategie om weerstand te kunnen bieden aan een persuasief verzoek. De belangrijkste resultaten van de onderzoeken in de drie empirische hoofdstukken van dit proefschrift worden hieronder besproken. 


\section{Hoofdstuk 2}

In het eerste hoofdstuk van dit proefschrift is een 2-fasen model geïntroduceerd, dat stelt dat zelfregulatie een onderliggende factor is die de effectiviteit van sociale beïnvloedingstechnieken zou kunnen verklaren. In Hoofdstuk 2 is de eerste fase van dit model getoetst. Verwacht werd dat de initiële fase van een sociale beïnvloedingstechniek, bestaande uit opeenvolgende verzoeken, zou leiden tot een verminderd vermogen tot het uitoefenen van zelfcontrole. Het beantwoorden van een of enkele initiële verzoeken of vragen wordt geacht ons vermogen tot actieve zelfregulatie uit te putten, omdat het vaak actieve zelfpresentatie, inspannende cognitieve handelingen of beide vereist (Schmeichel et al., 2003; Vohs et al., 2005). Vier experimenten toetsten deze hypothese, waarvan de eerste drie experimenten zijn uitgevoerd in het centrum van een grote stad. In Experiment 2.1 beantwoordden deelnemers een serie open vragen over hun gezondheid en leefstijl; een prototypische openingsfase van een sociale beïnvloedingstechniek die mensen er vermoedelijk toe aanzet een goede indruk te willen maken. Het beantwoorden van deze vragen leidde tot een hogere score op de State Ego Depletion Scale (Ciarocco et al., 2010); hetgeen getuigt van een minder goed vermogen tot zelfcontrole in vergelijking met een controleconditie waarin men niet werd gevraagd om deze vragen te beantwoorden. In Experimenten 2.2 en 2.3 is deelnemers opnieuw gevraagd om antwoord te geven op een serie persoonlijke vragen, maar ditmaal zijn personen in de controleconditie eveneens betrokken in een onverwachte interpersoonlijke interactie (van gelijke duur) met een voor hen onbekend persoon. Deelnemers in deze controleconditie is gevraagd om op een plattegrond van de stad de weg naar drie verschillende locaties aan te wijzen. In Experiment 2.2 induceerde het beantwoorden van een serie persoonlijke vragen opnieuw een verminderd vermogen tot zelfcontrole: deelnemers in deze conditie toonden minder doorzettingsvermogen in het oplossen van een (onoplosbare) puzzel dan deelnemers in de controleconditie. In Experiment 2.3 werd de alternatieve verklaring uitgesloten dat het schenden van de norm van wederkerigheid de eerdere 
resultaten zou kunnen verklaren: het beantwoorden van de initiële vragen leidde er niet toe dat men niet langer tegemoet wilde komen aan de persoon die de vragen stelde, in vergelijking met de controleconditie. Ook had het beantwoorden van de persoonlijke vragen geen (negatief) effect op emoties van de deelnemers of op de mate waarin ze de persoon die de vragen stelde aardig vonden. Wel gaf men aan een goede indruk te hebben willen maken, hetgeen zelfregulatie vereist (Vohs et al., 2005).

Deze eerste drie studies hebben laten zien dat de initiële fase van een sociale beïnvloedingstechniek die uit opeenvolgende verzoeken bestaat, leidt tot een verminderd vermogen tot het uitoefenen van zelfcontrole. Het 2-fasen model stelt echter dat een verminderd vermogen tot zelfregulatie functioneert als een mediator en zal leiden tot meer instemming met het doelverzoek van een beïnvloedingstechniek. Experiment 2.4 vervult een belangrijke rol, omdat het beide fasen van het model met elkaar verbindt. In deze studie zijn deelnemers blootgesteld aan een initieel verzoek, bestaande uit een serie cognitief inspannende vragen over de Belastingdienst. In de controleconditie beantwoordde men vergelijkbare, maar minder cognitief inspannende vragen. Het effect van het beantwoorden van deze vragen op instemming met het doelverzoek om deel te nemen aan toekomstige studies van de Belastingdienst werd naar verwachting gemedieerd door een verminderd vermogen tot zelfcontrole.

\section{Hoofdstuk 3}

In het tweede empirische hoofdstuk van dit proefschrift is de tweede fase van het 2-fasen model getoetst. Verwacht werd dat een tijdelijk verminderd vermogen tot zelfregulatie systematische informatieverwerking zou reduceren, waardoor men meer vertrouwt op heuristische informatieverwerking (zie ook Wheeler et al., 2007). Hierdoor zal de kans op instemming met een verzoek toenemen, mits een geschikte heuristiek aanwezig is in de beïnvloedingscontext. Vier experimenten toetsten deze hypothese. In Experiment 3.1 stemden deelnemers die eerder een aangeleerde respons moesten onderdrukken (zie 
ook Baumeister et al., 1998) in grotere mate in met een verzoek om vrijwillig deel te nemen aan toekomstig onderzoek; zij toonden zich bereid gedurende langere tijd hier aan deel te nemen dan personen in de controleconditie, wier vermogen tot zelfcontrole niet was aangetast. Belangrijk is dat een tijdelijk verminderd vermogen tot zelfregulatie op zichzelf niet leidde tot meer instemming; dit gebeurde alleen wanneer het heuristische principe van wederkerigheid saillant was gemaakt door deelnemers voorafgaand aan het verzoek een gunst te verlenen. In Experiment 3.2 doneerden deelnemers die eerder hun aandacht hadden gecontroleerd tijdens het bekijken van een video (Schmeichel et al., 2003) meer geld aan een goed doel dan deelnemers die zonder instructies naar de video hadden gekeken. Dit gebeurde echter alleen wanneer het goede doel een autoriteit was op het gebied van hulpverlening, in tegenstelling tot een minder bekend goed doel. In Experiment 3.3 werd het effect van zelfcontrole-uitputting op instemming met een verzoek eveneens gemodereerd door een heuristiek. Deelnemers die geometrische figuren hadden nagetekend met hun non-dominante hand, terwijl ze hun handbewegingen volgden via een spiegel (Quinn et al., 1996) waren in grotere getale bereid om deel te nemen aan toekomstig onderzoek van een organisatie dan deelnemers die de figuren met hun dominante hand en zonder spiegel hadden nagetekend. Dit effect trad echter alleen op wanneer de experimentleider hen gecomplimenteerd had met hun prestatie op de taak, om zo het heuristische principe van sympathie saillant te maken (zie ook Cialdini, 1993).

Wanneer het voorgestelde 2-fasen model algemeen geldend is, dan zou het door de voorgaande experimenten getoetste effect niet alleen moeten optreden wanneer een verminderd vermogen tot zelfregulatie situationeel geïnduceerd is, maar ook wanneer dit vermogen dispositioneel laag is (Tangney et al., 2004). In Experiment 3.4 bleken deelnemers met een dispositioneel laag vermogen tot zelfcontrole gevoeliger te zijn voor het heuristische principe van wederkerigheid: wanneer hen een gunst werd verleend door hun universiteit, toonden studenten zich bereid gedurende langere tijd onbetaald werk te verrichten voor hun universiteit dan deelnemers met een dispositioneel hoog vermogen tot zelfregulatie. 
Samen leveren de studies in Hoofdstukken 2 en 3 bewijs voor het 2-fasen model dat stelt dat zelfregulatie een onderliggende factor is die de effectiviteit van sociale beïnvloedingstechnieken zou kunnen verklaren. Wanneer het vermogen tot zelfcontrole is afgenomen tijdens de initiële fase van een sociale beïnvloedingstechniek blijkt men terug te vallen op heuristische informatieverwerking, waardoor de aanwezigheid van een geschikte heuristiek de kans vergroot dat men instemt met een verzoek tot het investeren van tijd, moeite of geld.

\section{Hoofdstuk 4}

Het laatste empirische hoofdstuk van dit proefschrift vormt een directe aanvulling op de veronderstellingen van het 2 -fasen model. Verwacht werd dat het vermogen tot zelfregulatie niet alleen zou kunnen verklaren waarom men onder bepaalde omstandigheden instemt met een verzoek, maar ook waarom men in bepaalde situaties weerstand biedt aan beïnvloeding. Waar een verminderd vermogen tot zelfregulatie gevoeliger maakt voor beïnvloeding, zal een hoog niveau van zelfregulatieve middelen de kans op beïnvloeding verkleinen. Maar is bij een verminderd vermogen tot zelfcontrole automatisch sprake van een verzwakte weerstand? Motivatie speelt hierbij mogelijk ook een belangrijke rol. Het onderzoek in Hoofdstuk 4 toont aan dat personen met een laag vermogen tot zelfregulatie zich desondanks goed kunnen verdedigen tegen een beïnvloedingspoging, wanneer zij gemotiveerd zijn om efficiënt om te gaan met hun resterende zelfregulatieve middelen. Wanneer sprake is van een verminderd vermogen tot zelfregulatie is men doorgaans niet volledig uitgeput, maar is dit vermogen slechts tijdelijk of relatief verminderd (Muraven et al., 2006). Men zou in dat geval nog steeds weerstand moeten kunnen bieden aan beïnvloeding, mits men tijdelijk bezuinigt op het gebruik van zelfregulatieve middelen en verdere uitputting voorkomt. Meer specifiek zou het vooraf waarschuwen dat men in de nabije toekomst blootgesteld zal worden aan een beïnvloedingspoging deze personen kunnen motiveren om hun resterende zelfregulatieve middelen te 'conserveren' door tijdelijk minder zelfcontrole uit te oefenen. Dit zal hen uiteindelijk in staat stellen om 
even succesvol te zijn in het bieden van weerstand aan beïnvloeding als personen die niet initieel uitgeput zijn. Een waarschuwing zal echter alleen effectief zijn voor mensen wier vermogen tot zelfcontrole tijdelijk is afgenomen, aangezien alleen zij behoefte zullen hebben aan het conserveren van zelfregulatieve middelen.

Drie experimenten toetsten de hypothese dat een waarschuwing dat men beïnvloed zal gaan worden ertoe aanzet meer weerstand te bieden aan beïnvloeding wanneer zelfregulatieve middelen tijdelijk zijn afgenomen. In Experiment 4.1 wilden deelnemers die eerder een aangeleerde respons moesten onderdrukken (vergelijk Experiment 3.1) minder tijd vrijmaken om vrijwillig collegezalen op te ruimen wanneer zij vooraf van dit komende verzoek op de hoogte waren gesteld. Zoals verwacht trad dit effect van waarschuwen niet op onder deelnemers van wie het vermogen tot zelfcontrole niet was aangetast. In een tweede experiment is het veronderstelde onderliggende proces getoetst: conservatie van resterende zelfregulatieve middelen. In Experiment 4.2 is voorafgaand aan het verzoek het conserveren van zelfregulatieve middelen vastgesteld met een zelfcontroletaak. Zoals verwacht motiveerde het vooraf waarschuwen de deelnemers met een tijdelijk verminderd vermogen tot zelfregulatie om hun resterende zelfregulatieve middelen te conserveren: zij presteerden minder goed op de zelfcontroletaak dan deelnemers in de overige condities. Evenals in Experiment 4.1 boden zij vervolgens evenveel weerstand aan beïnvloeding als deelnemers van wie het vermogen tot zelfcontrole niet was aangetast. Dit in tegenstelling tot deelnemers wier vermogen tot zelfcontrole was aangetast zonder hen vervolgens te waarschuwen. Zij waren niet aangespoord om efficiënt om te gaan met hun resterende zelfregulatieve middelen en boden aanzienlijk minder weerstand dan de deelnemers in de overige condities. Experiment 4.3 toonde opnieuw aan dat personen van wie het vermogen tot zelfcontrole tijdelijk is afgenomen meer weerstand bieden aan beïnvloeding wanneer zij vooraf gewaarschuwd zijn: zij genereerden meer argumenten tegen een persuasief verzoek dan niet-gewaarschuwde deelnemers omdat zij even daarvoor efficiënt waren omgegaan met hun resterende zelfregulatieve middelen. Hoewel het onderzoek in Hoofdstukken 2 en 3 heeft laten zien dat een tijdelijk 
verminderd vermogen tot zelfregulatie de kans vergroot dat men instemt met een persuasief verzoek, laat het onderzoek in Hoofdstuk 4 zien dat dit niet altijd het geval hoeft te zijn. Wanneer men gemotiveerd wordt om efficiënt om te gaan met resterende zelfregulatieve middelen is men even succesvol in het bieden van weerstand als personen wier vermogen tot zelfcontrole niet is aangetast.

\section{Algemene conclusie}

In dit proefschrift is een antwoord gezocht op de vraag waarom mensen vaak automatisch en gedachteloos reageren in beïnvloedingssituaties. Mensen blijken vaak met relatief weinig cognitieve inspanning te handelen wanneer zij worden blootgesteld aan een beïnvloedingstechniek en maken automatisch gebruik van heuristieken bij het nemen van een beslissing. De onderzoeksresultaten van dit proefschrift suggereren dat de wijze waarop mensen omgaan met (ongewenste) beïnvloeding voor een belangrijk deel afhankelijk is van de beschikbaarheid van middelen om actief controle uit te oefenen over het eigen gedrag. Daarbij is niet alleen het vermogen tot zelfregulatie belangrijk; ook de motivatie om efficiënt met zelfregulatieve middelen om te gaan blijkt bepalend te zijn voor de manier waarop mensen omgaan met persuasieve verzoeken. Ons vermogen en onze motivatie tot zelfcontrole blijken beide belangrijke voorspellers van de uitkomst van een sociale beïnvloedingssituatie. Door sociale beïnvloeding vanuit een perspectief van zelfregulatie te bekijken heeft dit proefschrift een mogelijk zeer invloedrijke psychologische determinant van beïnvloeding blootgelegd. Deze kennis kan degenen die zich beroepshalve bezig houden met beïnvloeding, maar bovenal de consument zelf ten goede komen. Met deze kennis op zak laat ik mij voortaan niet meer verleiden door een straatverkoper van de boekenclub. Optimaal gebruik makend van mijn zelfregulatieve vermogens koop ik mijn boeken gewoon zelf in de winkel. Dit neemt echter niet weg dat ik na een dag intensief winkelen misschien zomaar weer voor een andere verleiding bezwijk en bij het verlaten van de winkel mijn laatste geld afsta aan een goed doel. 



\section{Dankwoord}

(Acknowledgements in Dutch) 

E en van de dingen die dit proefschrift ons leert, is dat zelfcontrole een belangrijke rol speelt bij het nastreven van een doel. Dat je de controle soms ook even los moet laten om de batterij weer op te laden, is minstens even belangrijk. Volledig gefocust op dat eerste wilde ik het laatste nog wel eens vergeten. Gelukkig geeft het veel voldoening om jezelf te leren kennen, je angsten te overwinnen en uiteindelijk dat doel te bereiken. De afgelopen vier jaren waren een mooie tijd, waarvoor ik een groot aantal mensen op deze plek wil bedanken.

Ad Pruyn en Bob Fennis, mijn promotor en copromotor, ten eerste wil ik jullie bedanken dat ik mocht gaan promoveren op dit fantastische project.

Ad, als promotor heb je mij alle ruimte en vrijheid gegeven die ik nodig had om mijn plekje te kunnen vinden binnen de vakgroep Marketingcommunicatie en Consumenten Psychologie en me te kunnen ontwikkelen tot zelfstandig onderzoeker. Jij hebt ervoor gezorgd dat ik het einddoel niet uit het oog verloor en op mijn weg daar naartoe de juiste keuzes maakte. We hebben zakelijke en pittige gesprekken gevoerd, maar ik heb ook enorm gelachen om jouw lunchanekdotes en andere fratsen. Bedankt voor de fijne tijd bij MCP! Ook wil ik je vrouw Diane bedanken voor haar gastvrijheid tijdens de gezellige borrels en bbq's bij jullie thuis en voor het nauwkeurig editen van een deel van mijn proefschrift.

Bob, als dagelijks begeleider was je voor mij onmisbaar. Je hebt ontzettend veel energie en enthousiasme gestoken in de begeleiding van mijn project en daarvoor ben ik je erg dankbaar! Ook toen je in Utrecht ging werken, is dat uitstekend gegaan via e-mail en telefoon. Ik heb heel veel geleerd van je creativiteit en vakkennis, waar ik veel profijt van zal blijven hebben in mijn verdere carrière als wetenschapper. Daarbuiten heb ik ook erg genoten van jouw humor en zelfspot die verschillende congresbezoeken tot onvergetelijke uitstapjes maakten. Metha, ook jij bedankt voor die gezelligheid!

Uiteraard wil ik graag de leden van mijn promotiecommissie bedanken voor de tijd en moeite die ze hebben genomen voor het lezen van mijn proefschrift en voor hun aanwezigheid bij mijn verdediging. Ook gaat mijn dank uit naar alle studenten die op verschillende manieren een bijdrage hebben geleverd aan de studies in dit proefschrift. 
Kathleen, thank you so much for your contribution to this project. Working with you was inspiring and fun!

Mijn speciale dank gaat uit naar de collega's die mijn tijd in Twente tot een onvergetelijke tijd hebben gemaakt. Bij de vakgroep MCP staan de deuren altijd wagenwijd open. Karin, Marieke, Vanessa, Peter, Thomas, Joost, Martijn, Mirjam, Jos, Monique en Annet, jullie waren geweldige collega's! Ik kon altijd bij jullie terecht voor hulp en feedback, maar bovenal heb ik me dankzij jullie thuis gevoeld in Twente. Bedankt voor alle gezellige momenten. En dat waren er veel, heel veel.

Karin, toen wij samen een kamer gingen delen brak voor mij een andere tijd aan op de UT. 's Ochtends stond de roze theepot al klaar en kwamen de verhalen los. 'Hilarisch' is een standaard woord in mijn vocabulaire geworden. Bedankt voor alle steun en gezelligheid, tijdens en buiten het werk. Met je relativeringsvermogen als geen ander ben ik blij dat jij mijn paranimf bent!

Vanessa, Alexander en Jos, ook jullie waren fijne kamergenoten. Bedankt voor het aanhoren van mijn geklaag over vertraagde treinen en voor de fijne gesprekken. Vanes, het is uniek dat ik met jou mijn voorliefde voor foute rapnummers en ene James M. kan delen!

Marieke, je was een supercollega! Bedankt voor de gezellige tijd die ik met jou en Maarten heb beleefd tijdens congressen in het buitenland, en voor die ontelbare keren dat ik bij jullie in Enschede mocht blijven logeren.

Mijn andere UT-collega's, ook jullie wil ik graag bedanken voor de leuke tijd die ik met jullie heb gehad, tijdens aio-overleg, fietstochten, campuspop en de talloze etentjes, borrels en feestjes. Jullie zijn stuk voor stuk hele fijne mensen. In het bijzonder de meiden van de Jaaaarclub: Karin, Nicol, Renske, Fenne, Suzanne, Marieke, Nelly en Nicole: samen treinen, lunchwandelen, high-tea-en, sporten, shoppen, kleding ruilen, schrijven in een huisje op de hei, feestjes vieren en heel veel kletsen. Bedankt voor de gezelligheid!

Ook wil ik mijn collega's in Tilburg bedanken voor het warme onthaal op mijn nieuwe werkplek. Jullie adviezen en opbeurende woorden tijdens de laatste loodjes van dit proefschrift waren zeer welkom en ik vind het heel fijn dat ik bij jullie mijn werk mag voortzetten. 
Promoveren is mooi, maar het leven is nog veel mooier. Ook buiten het werk heb ik het geluk omringd te zijn met een heleboel fantastische mensen die mijn leven leuker maken. Met jullie kon ik de afgelopen jaren lief en leed delen en heb ik bovenal enorm veel plezier. Bedankt voor alle steun en de nodige afleiding en ontspanning! Hier wil ik de volgende mensen in het bijzonder noemen:

Shanti, onze vriendschap is ontstaan tijdens de laatste jaren van de middelbare school en sinds die tijd hebben we veel unieke momenten samen beleefd: op vakantie, tijdens werk en niet te vergeten tijdens al die uren die we dansend en feestend hebben doorgebracht! Die gezelligheid is er nog altijd, ook nu we de moezel met ijs steeds vaker verruilen voor een theetje op de bank. Weinig mensen zijn zo attent als jij en ik vind het fijn dat ik altijd bij je terecht kan. Bedankt dat je mijn paranimf wilt zijn!

Susanne, wat is het fijn om jou dicht in de buurt te hebben! En dat geldt ook voor Lieke en Ronald. Samen eten, sporten, naar theater en film, en heeeeel veel kletsen. Bij jullie kan ik heerlijk mezelf zijn.

Aefke en Hilde. De weekendjes op stap met jullie en Shanti zijn geweldig! Ik wed dat niemand ooit meer lol heeft gehad dan wij in Disneyland, Emmen en Volendam. Onze Sinterklaasfeesten met de andere meiden zijn legendarisch. Aef, bedankt voor de lieve opbeurende kaartjes die je me altijd stuurt!

Stefanie, Dieuwerke, Sabrina, Rinske, Marijke en Nienke, ook jullie wil ik bedanken voor de gezellige tijd die ik altijd met jullie heb en de fijne gesprekken de afgelopen jaren!

Marjolein en Claudia, heel belangrijk om af en toe even stoom af te blazen met het partyteam van Claudius. Bedankt voor de gezellige drankjes en etentjes.

Mijn lieve familie en schoonfamilie, jullie volgen mijn leven en carrière al jaren met veel interesse en trots. Ik vind het altijd fijn om weer even bij te praten en te ontspannen tijdens onze wandelingen, vakanties en andere gezellige bijeenkomsten. Frederic, we zien elkaar weinig, maar dankzij de telefoon zijn we gelukkig wel altijd op de hoogte van de laatste stand van zaken en leef je met me mee! 
Lieve paps en mams. Even pas op de plaats maken, denk je ook aan jezelf, ben je niet te druk. Dat heb ik jullie vaak horen zeggen de afgelopen jaren. Bezorgdheid zoals alleen jullie dat kunnen. En daarnaast de enorme trots, bij iedere vordering die ik maakte. Zo vaak zeg ik niet hoe belangrijk jullie voor mij zijn, maar voor jullie sta ik straks op dat podium!

Lieve Emiel, ik vind het bewonderenswaardig hoeveel jij altijd voor mij doet (en laat). Zonder jou had ik deze klus gewoonweg niet voor elkaar gekregen. Onvoorwaardelijke liefde en steun, zo kan ik het waarschijnlijk het beste omschrijven. Bedankt dat je er altijd voor mij bent. $X$.

Loes

4 januari, 2010 








